\title{
A GALEX ULTRAVIOLET IMAGING SURVEY OF GALAXIES IN THE LOCAL VOLUME
}

\author{
Janice C. Lee ${ }^{1,11}$, Armando Gil de Paz ${ }^{2}$, Robert C. Kennicutt, JR. ${ }^{3,4}$, Matthew Bothwell ${ }^{3}$, Julianne Dalcanton ${ }^{5}$, \\ José G. Funes, S. J. ${ }^{6}$, Benjamin D. Johnson ${ }^{3}$, Shoko SAKai $^{7}$, Evan Skillman ${ }^{8}$, Christy Tremonti $^{9}$, And Liese van Zee ${ }^{10}$ \\ ${ }^{1}$ Carnegie Observatories, 813 Santa Barbara Street, Pasadena, CA 91101, USA; jlee@ obs.carnegiescience.edu \\ ${ }^{2}$ Departamento de Astrofísica, Universidad Complutense de Madrid, Madrid 28040, Spain \\ ${ }^{3}$ Institute of Astronomy, University of Cambridge, Madingley Road, Cambridge, CB3 0HA, UK \\ ${ }^{4}$ Steward Observatory, University of Arizona, Tucson, AZ 85721, USA \\ ${ }^{5}$ Department of Astronomy, University of Washington, P.O. Box 351580, Seattle, WA 98195, USA \\ ${ }^{6}$ Vatican Observatory, Specola Vaticana, V00120 Vatican, Italy \\ ${ }^{7}$ Division of Astronomy and Astrophysics, University of California, Los Angeles, Los Angeles, CA 90095-1562, USA \\ ${ }^{8}$ Department of Astronomy, University of Minnesota, Minneapolis, MN 55455, USA \\ ${ }^{9}$ Department of Astronomy, University of Wisconsin-Madison, Madison, WI 53706, USA \\ ${ }^{10}$ Astronomy Department, Indiana University, Bloomington, IN 47405, USA \\ Received 2010 July 06; accepted 2010 October 30; published 2010 December 22
}

\begin{abstract}
We present results from a GALEX ultraviolet (UV) survey of a complete sample of 390 galaxies within $\sim 11 \mathrm{Mpc}$ of the Milky Way. The UV data are a key component of the composite Local Volume Legacy, an ultraviolet-toinfrared imaging program designed to provide an inventory of dust and star formation in nearby spiral and irregular galaxies. The ensemble data set is an especially valuable resource for studying star formation in dwarf galaxies, which comprise over $80 \%$ of the sample. We describe the GALEX survey programs that obtained the data and provide a catalog of far-UV $(\sim 1500 \AA)$ and near-UV $(\sim 2200 \AA)$ integrated photometry. General UV properties of the sample are briefly discussed. We compute two measures of the global star formation efficiency, the star formation rate (SFR) per unit $\mathrm{H}$ I gas mass, and the SFR per unit stellar mass, to illustrate the significant differences that can arise in our understanding of dwarf galaxies when the FUV is used to measure the SFR instead of H $\alpha$. We find that dwarf galaxies may not be as drastically inefficient in converting gas into stars as suggested by prior $\mathrm{H} \alpha$ studies. In this context, we also examine the UV properties of late-type dwarf galaxies that appear to be devoid of star formation because they were not detected in previous $\mathrm{H} \alpha$ narrowband observations. Nearly all such galaxies in our sample are detected in the FUV and have FUV SFRs that fall below the limit where the H $\alpha$ flux is robust to Poisson fluctuations in the formation of massive stars. Otherwise, the UV colors and star formation efficiencies of $\mathrm{H} \alpha$-undetected, UV-bright dwarf irregulars appear to be relatively unremarkable with respect to those exhibited by the general population of star-forming galaxies.
\end{abstract}

Key words: catalogs - galaxies: dwarf - galaxies: evolution - stars: formation - surveys - ultraviolet: galaxies

Online-only material: color figures, machine-readable tables

\section{INTRODUCTION}

Since the Galaxy Evolution Explorer (GALEX; Martin et al. 2005) was successfully launched in 2003, the study of star formation in the ultraviolet (UV) has been broadly enabled and expanded to regimes previously unexplored. In particular, GALEX far-UV (FUV; 1500 ̊) imaging has proved to be an exquisite probe of the recent star formation in relatively dustfree galactic environments that are characterized by low density and low-surface brightness.

A major theme that has emerged from work based on GALEX data is that there may be more star formation in low-density environments than previously recognized. For example, an early surprise from the mission was the detection of FUV emission extending to several times the optical disks of the nearby systems M83 (Thilker et al. 2005) and NGC 4625 (Gil de Paz et al. 2005), and it was reported that the amount and extent of the star formation in these "XUV disks" was greater than formerly seen in images of the $\mathrm{H} \alpha$ nebular emission line. Subsequent studies, which have begun to constrain the prevalence of XUV disks in the overall population, have indicated that extended star formation is common and occurs in roughly one-quarter to one-

\footnotetext{
${ }^{11}$ Carnegie Starr Fellow.
}

third of disk galaxies (Thilker et al. 2007; Zaritsky \& Christlein 2007; Goddard et al. 2010).

Later work investigated the globally integrated UV star formation rates (SFRs) of local dwarf and low-surface brightness galaxies, and several groups showed that, again, the amount of activity inferred from the UV in such systems tends to be greater than would be expected from previous $\mathrm{H} \alpha$ measurements (e.g., Meurer et al. 2009; Lee et al. 2009b; Boselli et al. 2009; Hunter et al. 2010). Our own study-based on the UV data set presented in this paper-was the first to establish this systematic trend with a statistically significant sample of dwarf galaxies with SFRs from $0.1 M_{\odot} \mathrm{yr}^{-1}$ down to $0.0001 M_{\odot} \mathrm{yr}^{-1}$ (Lee et al. 2009b). We find that at SFR $\sim 0.003 M_{\odot} \mathrm{yr}^{-1}$, the average $\mathrm{H} \alpha$-to-FUV flux ratio is lower than expected by about a factor of two, and at the lowest SFRs probed by our sample, the ratio exhibits up to an order-of-magnitude discrepancy. Prior to our work, there was a lack of deep UV observations for a complete, well-defined sample of dwarf galaxies, and this had prevented the trend from being clearly delineated in the past.

Until GALEX, a great deal of our knowledge of star formation in local galaxies had been based on $\mathrm{H} \alpha$ observations (e.g., Hodge 1974; Kennicutt 1989; Kennicutt et al. 2008, and references therein). From the recent GALEX results just summarized, however, it is now thought that the UV may be a more robust 
diagnostic of star formation activity in the low SFR intensity regime. UV emission should be less prone to stochastic effects from sparse sampling of the upper end of the stellar initial mass function (IMF) and to possible uncertainties in the fate of ionizing photons in low-density gas; that is, whether the H II regions, or even entire galaxies, can be safely assumed to be ionization-bounded (e.g., Melena et al. 2009). This is because the UV flux is directly emitted from the photospheres of O- through later-type B-stars $\left(M_{*} \gtrsim 3 M_{\odot}\right)$, whereas the $\mathrm{H} \alpha$ nebular emission arises from the recombination of hydrogen which can only be ionized by the most massive O- and early-type B-stars $\left(M_{*} \gtrsim 17 M_{\odot}\right)$. However, it is currently debated whether stochasticity or the potential leakage of Lyman continuum photons can fully account for the magnitude of the observed systematic between UV and $\mathrm{H} \alpha$ SFRs. A range of other causes have been considered (e.g., dust attenuation, metallicity, and discontinuous star formation histories), the most debated of which is an IMF deficient in the most massive stars (PflammAltenburg et al. 2009, and references therein; Meurer et al. 2009; Lee et al. 2009b; Boselli et al. 2009; Hunter et al. 2010). Though the explanation for the discrepancies between the two tracers is still uncertain, it is clear that UV observations from GALEX have challenged our understanding of star formation in low-density environments and are essential for the study of the outer disks of spirals, and of low-surface brightness and dwarf galaxies.

In this paper, we present results from a GALEX survey of a complete sample of Local Volume galaxies whose primary defining characteristic is that it is dominated by dwarf galaxiesover $80 \%$ of the sample have luminosities and SFRs lower than those of the Large Magellanic Cloud (LMC). This effort was undertaken as part of broader multi-wavelength campaign to provide new insight into the mechanisms that drive, regulate, and extinguish global star formation in galaxies, with special focus on the dwarf and low-surface brightness regime. Our survey has sought to build upon the patchwork of existing observations of nearby galaxies, which suffer from common observational biases toward luminous, high-surface brightness systems (e.g., Blanton et al. 2005). Though a great deal has already been learned about the nature of nearby dwarf star-forming galaxies, as low-metallicity, gas-rich, but not necessarily young systems (e.g., Gallagher \& Hunter 1984; van Zee 2001; Gil de Paz et al. 2003; Hunter \& Elmegreen 2004), the vast majority of prior studies have relied on observations of representative samples of dwarfs. Our work seeks to enable studies which demand dwarf galaxy samples that are not only representative, but are true to the statistics provided by an approximately volume-limited sample (e.g., characterization of the prevalence of starbursts in low-mass systems; Lee et al. 2009a).

$\mathrm{H} \alpha$ narrowband imaging observations for the sample were previously carried out by Kennicutt et al. (2008). The precursor $\mathrm{H} \alpha$ survey and GALEX follow-up program together comprise $11 \mathrm{HUGS}$, the $11 \mathrm{Mpc} \mathrm{H} \alpha$, and Ultraviolet Galaxy Survey. The data from 11HUGS have further been augmented by Spitzer IRAC mid-infrared and MIPS far-infrared observations (Dale et al. 2009) through the composite Local Volume Legacy ${ }^{12}$ program, which has provided the ensemble UV, $\mathrm{H} \alpha$, and IR data set to the community through the NASA/IPAC Infrared Science Archive $^{13}$ (IRSA). 11HUGS largely focuses on star-forming systems brighter than a certain limit $(B<15.5)$. To extend the coverage of the galaxy population to dwarf spheroidals, dwarf

\footnotetext{
12 http://www.ast.cam.ac.uk/research/lvls/

$13 \mathrm{http}: / / \mathrm{ssc}$. spitzer.caltech.edu/spitzermission/observingprograms/legacy/lvl/
}

ellipticals, and the faintest irregular galaxies, such systems from the ACS Nearby Galaxy Survey Treasury (ANGST) program (Dalcanton et al. 2009) were also targeted for both GALEX and Spitzer observations. ANGST has obtained Hubble Space Telescope (HST) resolved stellar population imaging for a volume-limited sample outside the Local Group and within $\sim 4$ Mpc. Altogether, the GALEX data set presented in this paper provides the most complete catalog of integrated UV photometry for Local Volume galaxies currently available.

The remainder of the paper is organized as follows. In Section 2, we describe in detail the resultant Local Volume sample selected for GALEX follow-up, summarize the observations, and present a catalog of NUV- and FUV-integrated photometry. In Section 3, basic results from the survey are presented. We give an overview of the UV properties of the sample and discuss the detection rate. We compute two measures of the global star formation efficiency, the SFR per unit $\mathrm{H}$ I gas mass and the SFR per unit stellar mass, to illustrate the significant differences that can arise in our understanding of dwarf galaxies when the FUV is used to measure the SFR instead of $\mathrm{H} \alpha$. In particular, the UV properties of late-type dwarf galaxies that appear to be devoid of star formation because they were not detected in previous $\mathrm{H} \alpha$ narrowband observations are examined. We comment on the selection of "transition" dwarf galaxies (dIrr/dSph) based upon the non-detection of $\mathrm{H} \alpha$ emission in gas-rich dwarfs. We adopt $H_{0}=75 \mathrm{~km} \mathrm{~s}^{-1} \mathrm{Mpc}^{-1}$ for distance-dependent quantities, when distance measurements from standard candles or secondary indicators are not available.

\section{DATA}

\subsection{Sample Selection}

The Local Volume galaxies that were targeted for GALEX imaging were mainly selected from the sample given in Kennicutt et al. (2008, hereafter Paper I), who have carried out a deep, statistically complete, $\mathrm{H} \alpha+[\mathrm{N}$ II] narrowband imaging survey within $\sim 11 \mathrm{Mpc}$. The ANGST program (Dalcanton et al. 2009), which has obtained HST ACS and WFPC2 imaging for a $\sim 4 \mathrm{Mpc}$ volume-limited sample, contains about 20 lowluminosity and/or early-type galaxies that were not already included in Paper I, and GALEX data were also obtained for these objects. In this section, we first summarize the Paper I sample and then describe the resultant set of galaxies that were observed by GALEX in our survey.

Paper I presents a sample of 436 galaxies from existing catalogs which were compiled in two components. The primary component aims to be as complete as possible in its inclusion of known nearby star-forming galaxies within given limits. It consists of all known spirals and irregulars $(T \geqslant 0)$ within 11 Mpc that avoid the Galactic plane $\left(|b|>20^{\circ}\right)$ and are brighter than $B=15$ mag. These particular limits represent the ranges within which the original surveys that have provided the bulk of our knowledge of the Local Volume galaxy population have been shown to be relatively complete (e.g., Tully 1988; de Vaucouleurs et al. 1991), but still span a large enough volume to probe a diverse cross-section of star formation properties. A secondary component consists of galaxies that are within $11 \mathrm{Mpc}$ for which $\mathrm{H} \alpha$ flux measurements are available, but fall outside one of the limits on brightness, Galactic latitude, and morphological type-these were generally targets either observed by our group as telescope time allowed or had $\mathrm{H} \alpha$ fluxes published in the literature. Subsequent statistical tests, as functions of the compiled $B$-band apparent magnitudes and 

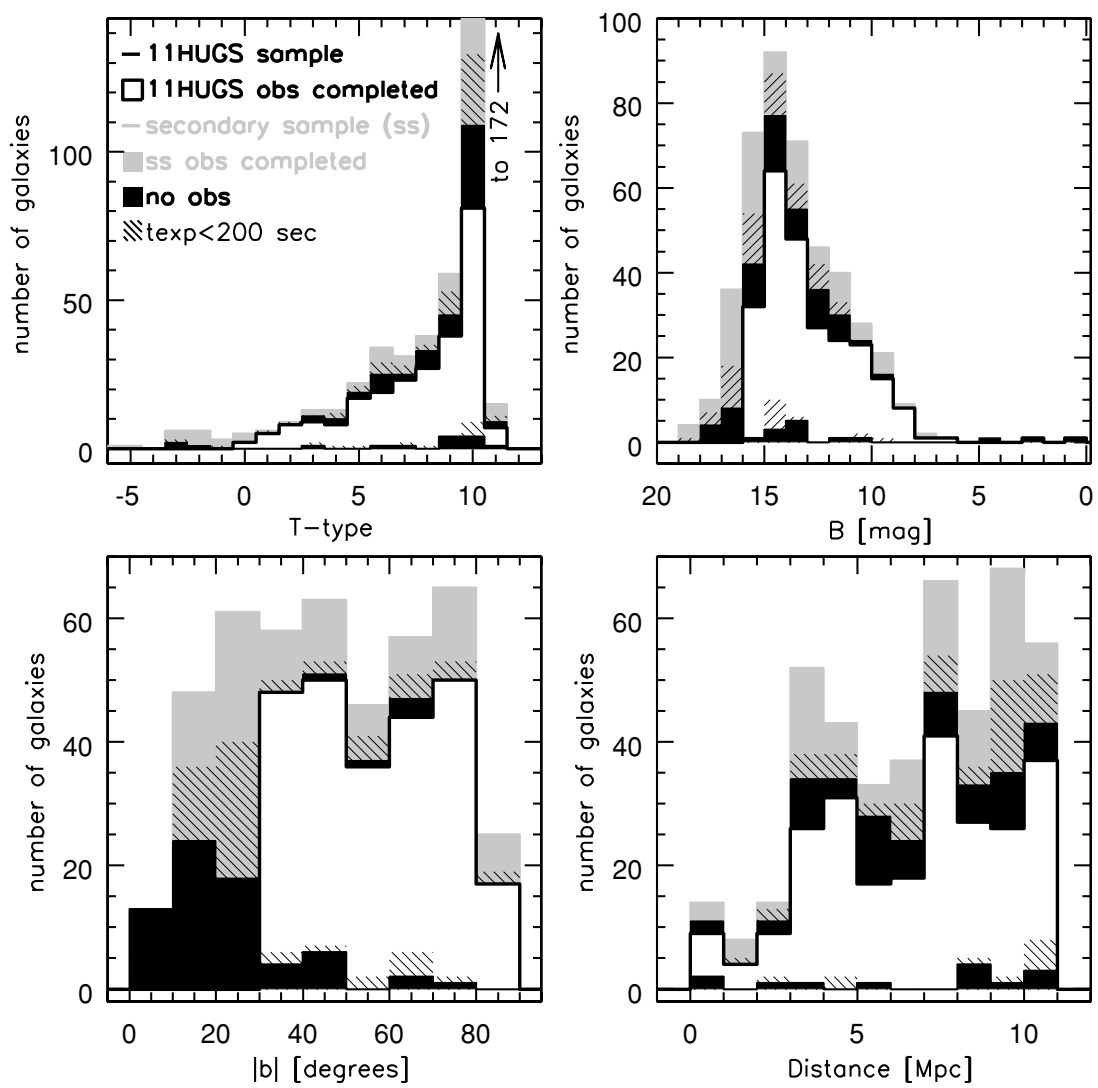

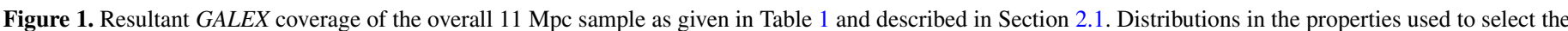

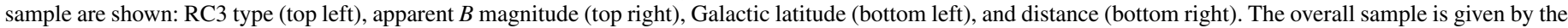

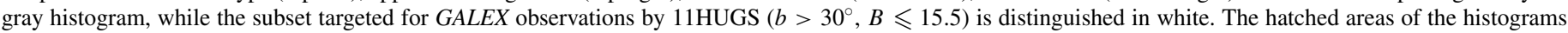

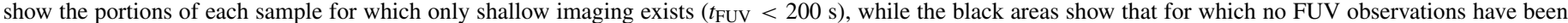
carried out. For $b>30^{\circ}$ and $B \leqslant 15.5$, there are no strong biases in the available one-orbit-depth $G A L E X$ imaging in any of these properties.

$21 \mathrm{~cm}(\mathrm{HI})$ fluxes, show that the overall sample is complete to $\sim 15.5 \mathrm{mag}$ and $\sim 6 \mathrm{Jy} \mathrm{km} \mathrm{s}^{-1}$, respectively. This corresponds to limits of $M_{B} \lesssim-15$ and $M_{\mathrm{H} \text { I }} \gtrsim 2 \times 10^{8} M_{\odot}$ for $|b|>20^{\circ}$ at the edge of the $11 \mathrm{Mpc}$ volume. More details on the parent sample and its properties are given in Paper I (sample construction, Local Volume membership uncertainties, and integrated $\mathrm{H} \alpha$ flux and equivalent width catalog), Lee et al. (2007; overall star formation demographics as traced by the $\mathrm{H} \alpha$ equivalent width), and Lee et al. (2009a; completeness tests and limits, and dwarf galaxy starburst statistics).

Galaxies with $|b|>30^{\circ}$ and $B \leqslant 15.5$ from the Paper I sample $(N=256)$ were targeted for UV imaging through the 11HUGS GALEX Legacy program (GI1_047, GI4_095). The Galactic latitude limit was imposed to avoid excessive foreground extinction, and fields with bright stars and/or high background levels for which imaging would be prohibited due to the instrument's brightness safety limits. GALEX observations for a significant number $(N=120)$ of the remaining lower latitude, fainter galaxies in the parent sample have also been taken by other programs and are publicly available in the GALEX archive through MAST. ${ }^{14}$ We have performed photometry on

\footnotetext{
14 MAST is the Multimission Archive at the Space Telescope Science Institute (http://archive.stsci.edu/index.html). STScI is operated by the Association of Universities for Research in Astronomy, Inc., under NASA contract NAS5-26555. Support for MAST for non-HST data is provided by the NASA Office of Space Science via grant NAG5-7584 and by other grants and contracts.
}

these data as well and provide measurements in the tables that follow.

Observations of the 11HUGS galaxies have also been extended into the infrared using the Spitzer Space Telescope as part of the Local Volume Legacy survey (Dale et al. 2009). As mentioned above, in addition to galaxies selected from Paper I, Local Volume Legacy also includes targets from ANGST in order to extend coverage of galaxy properties to dwarf spheroidal and ellipticals, and the faintest dwarf irregulars known outside of the Local Group. There are 27 galaxies in the composite Local Volume Legacy sample which are not included in Paper I. ${ }^{15} G A L E X$ observations have also been obtained for all of these galaxies, about half of which were taken through a GALEX follow-up program of ANGST galaxies (GI3_06). The photometry for these 27 galaxies is also reported here.

\subsection{GALEX Observations}

GALEX is a NASA Small Explorer launched in 2003 April. It uses a $50 \mathrm{~cm}$ aperture telescope with a dichroic beam splitter that enables simultaneous observations in the FUV $\left(\lambda_{\text {eff }}=1539 \AA, F W H M=269 \AA\right)$ and the NUV $\left(\lambda_{\text {eff }}=2316 \AA\right.$, FWHM $=616 \AA)$. The field of view of the camera is circular and has a 1.2 diameter. The PSF is dependent on the count rate (deteriorates for brighter sources) as well as on the radial

\footnotetext{
15 Included in this group of 27 are 4 galaxies for which a revision in the distance estimates placed them outside $11 \mathrm{Mpc}$ after the final sample for Local Volume Legacy Spitzer observations had been selected (see Section 2.2).
} 
position on the image (deteriorates at the field edge), but is reasonably well described by a FWHM of $5^{\prime \prime} \pm 1^{\prime \prime}$ for the data analyzed here. The images that we use have been uniformly reprocessed by the GALEX team with the most recent version of the pipeline (v6) available at the time of the initial preparation of this paper. The 6 pipeline accounts for a drift in the photometric calibration $(1.5 \%$ and $0.25 \%$ dimmer per year for the NUV and FUV bands, respectively), incorporates corrections to the flat-fielding (5\% systematic in the FUV and a $2 \%$ change in shape in the NUV), and improves the removal of reflected light at the NUV field edge. Full details on the satellite, telescope, instrument and calibration, and data processing pipeline are given in Martin et al. (2005), Morrissey et al. (2005), and Morrissey et al. (2007).

Our primary survey objective is to provide a statistically complete UV data set for a deep Local Volume galaxy sample. To do this, our program leverages upon existing data and homogeneously fills significant gaps in prior GALEX coverage of the $D<11 \mathrm{Mpc}, b>30^{\circ}, B<15.5$ population $(N=$ 256). The GALEX team's Nearby Galaxy Survey and Medium Imaging Survey has supplied imaging for $\sim 30 \%$ of this sample (Gil de Paz et al. 2007), with cumulative exposure times of $\sim 1500$ s per field (close to the time available in one orbit), while a small fraction $(\sim 10 \%)$ were observed to similar depth by other independent guest investigator programs. This exposure time corresponds to surface brightness limits of $m_{\mathrm{AB}} \sim$ $27.5 \mathrm{mag} \operatorname{arcsec}^{-2}$ or an SFR of $\sim 10^{-3} M_{\odot} \mathrm{yr}^{-1} \mathrm{kpc}^{-2}$ (Martin et al. 2005; Gil de Paz et al. 2007). Our Cycle 1 (GI1047) and Cycle 4 (GI4-095) programs obtained one-orbit-depth data for an additional $\sim 50 \%$ of the sample. The status of GALEX observations for the remaining of $\sim 10 \%$ of this sample is as follows. Observations for 10 galaxies are prohibited due to bright foreground stars and/or high background (LMC, SMC, ISZ399, NGC1313, NGC3077, NGC7713, UGC5076, UGC7490, UGC8508, and UGC8837). ${ }^{16}$ Observations for 10 galaxies have not been completed to sufficient depth in the FUV (i.e., less than $1 / 3$ the requested integration time or $500 \mathrm{~s}$ ), and only shallow imaging from the GALEX All-sky Imaging Survey (AIS) is available (IC625, IC2782, IC4316, NGC3675, NGC5608, UGC5451, UGC8091, UGC9128, UGC9211, and UGC9660). ${ }^{17}$ No data are available for four galaxies (ESO252IG001, ESO306-G013, UGC5151, and UGCA103). Overall, observations for the ensemble data set were taken over five years between 2004 and 2009. Intermittent FUV detector failures, or "transient overcurrent events," that have occurred since 2004 (Morrissey 2006), caused the completion of the survey to require more time than initially anticipated.

UV imaging is also available from other GALEX programs for another 120 of the remaining 180 galaxies in Paper I which fall outside the limits of the main $b>30^{\circ}$ and $B \leqslant 15.5$ sample. Approximately half of these galaxies have shallow AIS imaging $(\lesssim 200$ s), while the remainder have deep $(\geqslant 1500$ s) exposures.

Figure 1 summarizes the resultant GALEX coverage of the overall $11 \mathrm{Mpc}$ sample as described above. Distributions of

\footnotetext{
16 Although observations for fields close to NGC1313 and NGC3077 are currently not allowed, some early data were taken by the GALEX team for these galaxies. A shallow All-sky Imaging Survey (AIS) $211 \mathrm{~s}$ pointing exists for NGC1313. Observations of NGC3077 were attempted by the Nearby Galaxy Survey, but in the effort to shift the offending bright stars off of the GALEX FOV, the galaxy was moved too close to the field edge, and only about half of it was captured in the resultant imaging.

17 Two of these galaxies, IC2782 and UGC9128, have deep (>1500 s) exposures in the NUV, though their FUV data are shallow. This is a consequence of intermittent FUV detector failures, during which observations still proceeded in the NUV channel.
}

the morphological type, $B$ magnitude, Galactic latitude, and distance (i.e., the properties used to define our samples) are shown. The overall $11 \mathrm{Mpc}$ parent sample (Table 1) is given by the gray histogram, while the subset selected for GALEX observations by $11 \mathrm{HUGS}\left(b>30^{\circ}, B \leqslant 15.5\right)$ is distinguished in white. The hatched portions of the histograms show the fractions of each sample for which only shallow imaging exists $\left(t_{\mathrm{FUV}}<200 \mathrm{~s}\right)$, while the black portions show those for which no FUV observations have been carried out. There are no strong biases in the available one-orbit-depth GALEX imaging in any of these properties.

Table 1 lists specific exposure times and information on the original GALEX programs that obtained the data, along with general properties of the targets. To facilitate cross-comparison with the $\mathrm{H} \alpha$ data presented in Paper I, basic properties given in the first table of Paper I are repeated in Columns 1-9 with some updates, and all 436 galaxies are listed, whether they have been observed by GALEX or not. Galaxies in the composite Local Volume Legacy sample which are not included in Paper I (generally targets drawn from the ANGST sample) are listed separately at the end of the table. The columns in Table 1 are as follows.

Column 1: the running index number.

Column 2: galaxy name.

Columns 3 and 4: J2000 right ascension and declination as reported in NASA/IPAC Extragalactic Database (NED).

Column 5: galactic latitude as reported in NED.

Column 6: adopted distance. Measurements based on standard candles or secondary distance indicators were compiled from the literature when available, otherwise distances computed from recessional velocities corrected to the centroid of the Local Group (following Karachentsev \& Makarov 1996, as reported in NED) and adopting $H_{0}=75 \mathrm{~km} \mathrm{~s}^{-1} \mathrm{Mpc}^{-1}$ are listed. Original references for the direct distances are given in Paper I, Table 1, with the exception of galaxies targeted by the ANGST program, for which non-Cepheid distances have been updated to be consistent with the tip of the red giant branch measurements reported in Dalcanton et al. (2009).

Column 7: distance determination method, with the following abbreviations: Cepheid variables (ceph), tip of the red giant branch (trgb), surface brightness fluctuations (sbf), membership in a group with measured distance (mem), brightest blue stars (bs), Tully-Fisher relation (tf), and Local Group corrected recessional velocity distances (flow).

Column 8: apparent $B$-band magnitude. The photometry was compiled as discussed in Lee et al. (2009a), and references are given in Paper I, Table 1.

Column 9: RC3 morphological type.

Column 10: a flag indicating whether the galaxy is included in the Spitzer Local Volume Legacy program (1) or not (0). The same $|b|>30^{\circ}, B<15.5$ Local Volume population was targeted for both GALEX and Spitzer (IRAC 3.6, 4.5, 5.8, and $8.0 \mu \mathrm{m}$ and MIPS 24, 70, and $160 \mu \mathrm{m}$ ) follow-up. However, changes in the adopted distances for some galaxies which occurred between the selection of the Spitzer and final GALEX/ Paper I samples led to slight differences between the samples. This flag is provided here to help clarify these differences. As described in Dale et al. (2009), four galaxies targeted for Spitzer observations have updated distances which place them outside of $11 \mathrm{Mpc}$. These galaxies appear at the end of the table. The flow model initially applied was also updated to provide consistency with one used by NED. As a result, 30 Paper I galaxies with $|b|>30^{\circ}, B<15.5$ do not have Spitzer imaging in Dale et al. (2009). The galaxies are generally between 10 
Table 1

GALEX Observations of Local Volume Galaxies

\begin{tabular}{|c|c|c|c|c|c|c|c|c|c|c|c|c|}
\hline No. & $\begin{array}{c}\text { Galaxy Name } \\
(2) \\
\end{array}$ & $\begin{array}{c}\text { R.A. } \\
(\mathrm{J} 2000) \\
(3)\end{array}$ & $\begin{array}{c}\text { Decl. } \\
(\mathrm{J} 2000) \\
(4) \\
\end{array}$ & $\begin{array}{c}b \\
(\mathrm{deg}) \\
(5) \\
\end{array}$ & $\begin{array}{c}\mathrm{D} \\
(\mathrm{Mpc}) \\
(6) \\
\end{array}$ & $\begin{array}{c}\text { Method } \\
\text { (7) }\end{array}$ & $\begin{array}{c}B \\
(\mathrm{mag}) \\
(8) \\
\end{array}$ & $(9)$ & $\begin{array}{l}\text { Local Volume Legacy } \\
\text { (10) } \\
\end{array}$ & $\begin{array}{c}t_{\text {fuv }} \\
(\mathrm{s}) \\
(11) \\
\end{array}$ & $\begin{array}{l}t_{\text {nuv }} \\
(\mathrm{s}) \\
(12) \\
\end{array}$ & $\begin{array}{c}\text { Tile Name } \\
\text { (13) }\end{array}$ \\
\hline 1 & UGC12894 & 000022.5 & 392944 & -22.32 & 8.2 & $\mathrm{v}$ (flow) & 16.6 & 10 & 0 & & & \\
\hline 2 & WLM & 000158.1 & -152739 & -73.63 & 0.92 & $\operatorname{trgb}$ & 11.03 & 10 & 1 & 1440 & 1440 & NGA_WLM \\
\hline 3 & ESO409-IG015 & 000531.8 & -280553 & -79.79 & 10.4 & $\mathrm{v}$ (flow) & 15.1 & 6 & 0 & 1608 & 1608 & GI1_009016_HPJ0005m28 \\
\hline 4 & ESO349-G031 & 000813.3 & -343442 & -78.12 & 3.21 & $\operatorname{trgb}$ & 15.6 & 10 & 0 & 1608 & 1608 & GI1_047001_ESO349_G031 \\
\hline 5 & NGC24 & 000956.7 & -245744 & -80.43 & 8.1 & $\mathrm{v}$ (flow) & 12.2 & 5 & 1 & 1579 & 1579 & NGA_NGC0024 \\
\hline 6 & NGC45 & 001404.0 & -231055 & -80.67 & 7.1 & $\mathrm{v}$ (flow) & 11.32 & 8 & 1 & 2220 & 2220 & GI4_095001_NGC0045 \\
\hline 7 & NGC55 & 001454.0 & -391149 & -75.74 & 2.10 & $\operatorname{trgb}$ & 8.42 & 9 & 1 & 1482 & 1482 & NGA_NGC0055 \\
\hline 8 & NGC59 & 001525.4 & -212642 & -80.02 & 5.3 & $\mathrm{sbf}$ & 13.1 & -3 & 1 & 2393 & 2393 & GI4_095002_NGC0059 \\
\hline 9 & MCG-04-02-003 & 001911.4 & -224006 & -81.44 & 9.8 & $\mathrm{v}$ (flow) & 15.6 & 9 & 0 & 1655 & 3429 & GI1_047003_MC̄G_04_02_003 \\
\hline 10 & IC10 & 002023.1 & 591735 & -3.34 & 0.66 & ceph & 11.8 & 10 & 0 & & & \\
\hline 11 & ESO473-G024 & 003122.5 & -224557 & -83.70 & 8.0 & $\mathrm{v}$ (flow) & 16.04 & 10 & 0 & 1600 & 1600 & GI1_009007_HPJ0031m22 \\
\hline 12 & AndIV & 004230.1 & 403433 & -22.27 & 6.11 & $\operatorname{trgb}$ & 16.60 & 10 & 0 & 3562 & 3562 & NGA_M31_MOS12 \\
\hline 13 & NGC224 & 004244.3 & 411609 & -21.57 & 0.79 & ceph & 4.36 & 3 & 0 & $\ldots$ & $\ldots$ & \\
\hline 14 & IC1574 & 004303.8 & -221449 & -84.76 & 4.92 & $\operatorname{trgb}$ & 14.50 & 10 & 1 & 1626 & 1627 & GI4_095043_IC1574 \\
\hline 15 & NGC247 & 004708.3 & -204538 & -83.56 & 3.52 & $\operatorname{trgb}$ & 9.67 & 7 & 1 & 2507 & 2507 & NGA_NGC̄0247 \\
\hline 16 & NGC253 & 004733.1 & -251718 & -87.96 & 3.44 & $\operatorname{trgb}$ & 8.04 & 5 & 1 & 3272 & 3272 & NGA_NGC0253 \\
\hline 17 & UGCA15 & 004949.2 & -210054 & -83.88 & 3.31 & $\operatorname{trgb}$ & 15.4 & 10 & 1 & 1513 & 1513 & GI1_009002_UGCA015 \\
\hline 18 & SMC & 005244.8 & -724943 & -44.33 & 0.06 & ceph & 2.70 & 9 & 1 & & & \\
\hline 19 & NGC300 & 005453.5 & -374100 & -79.42 & 2.00 & ceph & 8.72 & 7 & 1 & 1628 & 1628 & NGA_NGC0300 \\
\hline 20 & LGS3 & 010355.0 & 215306 & -40.89 & 0.62 & $\operatorname{trgb}$ & 16.18 & 99 & 0 & 1700 & 1700 & NGA_LGS3 \\
\hline 21 & UGC668 & 010447.8 & 020704 & -60.56 & 0.65 & ceph & 9.88 & 10 & 1 & 1696 & 1696 & NGA_IC1613 \\
\hline 22 & UGC685 & 010722.4 & 164102 & -46.02 & 4.70 & $\operatorname{trgb}$ & 14.20 & 9 & 1 & 1609 & 1609 & GI1_047004_UGC00685 \\
\hline 23 & UGC695 & 010746.4 & 010349 & -61.53 & 10.2 & $\mathrm{v}$ (flow) & 15.3 & 6 & 1 & 1635 & 1635 & GI1_047005_UGC00695 \\
\hline 24 & UGC891 & 012118.9 & 122443 & -49.80 & 10.8 & $\mathrm{v}$ (flow) & 14.72 & 9 & 1 & 2685 & 4333 & GI1_047006_UGC00891 \\
\hline 25 & UGC1056 & 012847.3 & 164119 & -45.26 & 10.3 & $\mathrm{v}$ (flow) & 14.9 & 10 & 1 & 2620 & 2620 & GI4_095003_UGC01056 \\
\hline 26 & UGC1104 & 013242.5 & 181902 & -43.47 & 7.5 & bs & 14.41 & 9 & 1 & 1168 & 2864 & GI1_036006_UGC01104 \\
\hline 27 & NGC598 & 013350.9 & 303937 & -31.33 & 0.84 & ceph & 6.27 & 6 & 1 & 3361 & 3361 & NGA_M33_MOS0 \\
\hline 28 & NGC625 & 013504.2 & -412615 & -73.12 & 4.07 & $\operatorname{trgb}$ & 11.7 & 9 & 1 & 4530 & 4530 & GI1_047007_NGC0625 \\
\hline 29 & NGC628 & 013641.7 & 154659 & -45.71 & 7.3 & bs & 9.95 & 5 & 1 & 1636 & 1636 & NGA_NGC0628 \\
\hline 30 & UGC1176 & 014009.9 & 155417 & -45.37 & 9.0 & bs & 14.4 & 10 & 1 & 1696 & 3200 & GI1_047008_UGC01176 \\
\hline 31 & UGCA20 & 014314.7 & 195832 & -41.25 & 9.0 & $\mathrm{v}$ (flow) & 15.78 & 10 & 0 & 3382 & 5078 & GI1_047010_UGCA020 \\
\hline 32 & ESO245-G005 & 014503.7 & -433553 & -70.29 & 4.43 & $\operatorname{trgb}$ & 12.7 & 10 & 1 & 2424 & 2424 & GI1_047011_ESO245_G005 \\
\hline 33 & UGC1249 & 014730.6 & 271952 & -33.90 & 7.2 & bs & 12.1 & 9 & 1 & 1526 & 3097 & GI1_047012_IC1727 \\
\hline 34 & NGC672 & 014754.3 & 272559 & -33.78 & 7.2 & bs & 11.47 & 6 & 1 & 1526 & 3097 & GI1_047012_IC1727 \\
\hline 35 & UGC1281 & 014931.4 & 323519 & -28.71 & 5.13 & $\operatorname{trgb}$ & 13.61 & 8 & 0 & 137 & 137 & AISCHV4_058_06877 \\
\hline 36 & ESO245-G007 & 015106.3 & -442641 & -68.95 & 0.44 & $\operatorname{trgb}$ & 13.3 & 10 & 1 & 1702 & 1702 & NGA_Phoenix \\
\hline 37 & NGC784 & 020117.0 & 285015 & -31.59 & 5.19 & $\operatorname{trgb}$ & 12.23 & 8 & 1 & 2267 & 2267 & GI4_095004_NGC0784 \\
\hline 38 & UGC1561 & 020405.1 & 241230 & -35.75 & 10.5 & $\mathrm{v}$ (flow) & 16.2 & 10 & 0 & 1693 & 1693 & GI1_047013_UGC01561 \\
\hline 39 & NGC855 & 021403.6 & 275238 & -31.53 & 9.7 & sbf & 13.3 & -4 & 1 & 1500 & 1500 & NGA_NGC0855 \\
\hline 40 & UGC1807 & 022113.4 & 424546 & -17.12 & 9.2 & mem & 16.50 & 10 & 0 & 7355 & 7667 & GI2_019004_3C66B \\
\hline 41 & NGC891 & 022233.4 & 422057 & -17.41 & 9.2 & mem & 10.8 & 3 & 0 & 1696 & 1696 & NGA_NGC0891 \\
\hline 42 & UGC1865 & 022500.2 & 360216 & -23.10 & 9.2 & mem & 14.4 & 9 & 0 & 169 & 169 & AISCHV4_059_06618 \\
\hline 43 & NGC925 & 022716.9 & 333445 & -25.17 & 9.16 & ceph & 10.7 & 7 & 0 & 1671 & 1671 & NGA_NGC0925 \\
\hline 44 & UGC1924 & 022749.8 & 314336 & -26.82 & 10.4 & $\mathrm{v}$ (flow) & 15.23 & 6 & 0 & 144 & 144 & AISCHV3_064_07003 \\
\hline 45 & NGC949 & 023048.6 & 370814 & -21.63 & 9.2 & mem & 12.4 & 4 & 0 & 181 & 181 & AISCHV4_059_06618 \\
\hline 46 & NGC959 & 023224.0 & 352942 & -23.00 & 9.2 & mem & 12.9 & 8 & 0 & 1694 & 1694 & NGA_NGC0959 \\
\hline 47 & UGC2014 & 023254.0 & 384050 & -20.05 & 9.2 & mem & 15.7 & 10 & 0 & 170 & 170 & AISCHV4_059_06618 \\
\hline 48 & UGC2023 & 023318.2 & 332928 & -24.74 & 9.2 & mem & 13.9 & 10 & 0 & 108 & 108 & AISCHV4_064_06804 \\
\hline 49 & UGC2034 & 023342.9 & 403141 & -18.29 & 9.2 & mem & 13.7 & 10 & 0 & $\ldots$ & $\ldots$ & $\ldots$ \\
\hline 50 & ESO115-G021 & 023748.1 & -612018 & -51.43 & 4.99 & $\operatorname{trgb}$ & 13.34 & 8 & 1 & 2220 & 2220 & GI4_095005_ESO115_G021 \\
\hline 51 & NGC1003 & 023916.6 & 405221 & -17.54 & 9.2 & mem & 12.00 & 6 & 0 & 114 & 114 & AISCHV4_059_06440 \\
\hline 52 & Maffei2 & 024154.9 & 593615 & -0.33 & 3.3 & $\mathrm{tf}$ & 14.8 & 4 & 0 & $\ldots$ & $\ldots$ & $\ldots$ \\
\hline 53 & NGC1058 & 024329.9 & 372027 & -20.37 & 9.2 & mem & 11.8 & 5 & 0 & 2778 & 2778 & GI3_067002_SN2007gr_p \\
\hline 54 & UGC2259 & 024755.4 & 373218 & -19.80 & 9.2 & mem & 15.2 & 8 & 0 & $\ldots$ & $\ldots$ & $\ldots$ \\
\hline 55 & ESO154-G023 & 025650.4 & -543417 & -54.31 & 5.76 & $\operatorname{trgb}$ & 12.69 & 8 & 1 & 1573 & 1573 & GI4_095006_ESO154_G023 \\
\hline 56 & NGC1156 & 025942.6 & 251417 & -29.20 & 7.8 & bs & 12.75 & 10 & 0 & 1423 & 1423 & NGA_NGC1156 \\
\hline 57 & ESO300-G014 & 030937.8 & -410150 & -58.46 & 11.0 & $\mathrm{v}$ (flow) & 13.0 & 9 & 0 & 1640 & 1640 & GI1_047015_ESO300_G014 \\
\hline 58 & ESO300-G016 & 031010.5 & -400011 & -58.62 & 7.8 & $\mathrm{v}$ (flow) & 15.6 & 10 & 0 & 1646 & 1646 & GI1_009006_NGC1249 \\
\hline 59 & NGC1291 & 031718.3 & -410628 & -57.05 & 9.4 & $\mathrm{v}$ (flow) & 9.39 & 0 & 1 & 1705 & 1705 & NGA_NGC1291 \\
\hline 60 & NGC1313 & 031815.8 & -662953 & -44.64 & 4.15 & $\operatorname{trgb}$ & 9.2 & 7 & 1 & 211 & 211 & AISCHV4_393_39112 \\
\hline 61 & NGC1311 & 032007.4 & -521107 & -52.66 & 5.45 & $\operatorname{trgb}$ & 13.18 & 9 & 1 & 1855 & 1855 & MIS2DFR_38632_0859 \\
\hline 62 & UGC2684 & 032023.7 & 171745 & -32.75 & 6.5 & bs & 16.30 & 10 & 0 & 4065 & 6692 & GI1_047018_UGC02684 \\
\hline 63 & UGC2689 & 032127.7 & 404806 & -13.68 & 5.9 & $\mathrm{v}$ (flow) & 14.95 & -2 & 0 & $\ldots$ & $\ldots$ & $\ldots$ \\
\hline
\end{tabular}


Table 1

(Continued)

\begin{tabular}{|c|c|c|c|c|c|c|c|c|c|c|c|c|}
\hline No. & $\begin{array}{c}\text { Galaxy Name } \\
\text { (2) }\end{array}$ & $\begin{array}{c}\text { R.A. } \\
(\mathrm{J} 2000) \\
(3)\end{array}$ & $\begin{array}{c}\text { Decl. } \\
(\mathrm{J} 2000) \\
(4)\end{array}$ & $\begin{array}{c}b \\
(\operatorname{deg}) \\
(5) \\
\end{array}$ & $\begin{array}{c}\mathrm{D} \\
(\mathrm{Mpc}) \\
(6)\end{array}$ & $\begin{array}{l}\text { Method } \\
\text { (7) }\end{array}$ & $\begin{array}{c}B \\
(\mathrm{mag}) \\
(8)\end{array}$ & $(9)$ & $\begin{array}{l}\text { Local Volume Legacy } \\
\qquad(10)\end{array}$ & $\begin{array}{l}t_{\mathrm{fuv}} \\
(\mathrm{s}) \\
(11)\end{array}$ & $\begin{array}{l}t_{\text {nuv }} \\
(\mathrm{s}) \\
(12) \\
\end{array}$ & $\begin{array}{l}\text { Tile Name } \\
\text { (13) }\end{array}$ \\
\hline 64 & UGC2716 & 032407.2 & 174512 & -31.82 & 6.2 & $\mathrm{v}$ (flow) & 14.64 & 8 & 1 & 4065 & 6692 & GI1_047018_UGC02684 \\
\hline 65 & IC1959 & 033311.8 & -502438 & -51.54 & 6.06 & $\operatorname{trgb}$ & 13.26 & 9 & 1 & 1504 & 1504 & GI1_047019_IC1959 \\
\hline 66 & UGC2847 & 034648.9 & 680546 & 10.58 & 3.03 & ceph & 9.37 & 6 & 0 & 4466 & 5747 & LGAL_IC342 \\
\hline 67 & IC2000 & 034907.3 & -485131 & -49.60 & 10.7 & $\mathrm{v}$ (flow) & 13 & 6 & 0 & 544 & 544 & MIS2DFR_38660_0817 \\
\hline 68 & ESO302-G014 & 035140.9 & -382708 & -50.88 & 9.6 & $\mathrm{v}$ (flow) & 16 & 10 & 0 & 1696 & 1696 & GI1_047021_ESO302_G014 \\
\hline 69 & NGC1487 & 035546.1 & -422205 & -49.76 & 9.1 & $\mathrm{v}$ (flow) & 12.34 & 7 & 1 & 1696 & 1696 & GI1_047022_NGC1487 \\
\hline 70 & ESO249-G036 & 035915.6 & -455221 & -48.61 & 9.6 & $\mathrm{v}$ (flow) & 15.8 & 10 & 0 & 1501 & 3095 & GI1_047023_HorDwarf \\
\hline 71 & UGCA86 & 035950.1 & 670837 & 10.65 & 2.96 & trgb & 13.50 & 10 & 0 & 155 & 155 & AISCHV4_052_05261 \\
\hline 72 & NGC1510 & 040332.6 & -432400 & -48.23 & 9.8 & $\mathrm{v}$ (flow) & 13.5 & -2 & 1 & 2370 & 2370 & NGA_NGC1512 \\
\hline 73 & NGC1512 & 040354.3 & -432057 & -48.16 & 9.6 & $\mathrm{v}$ (flow) & 11.13 & 1 & 1 & 2370 & 2370 & NGA_NGC1512 \\
\hline 74 & NGC1522 & 040607.7 & -524009 & -45.97 & 9.3 & $\mathrm{v}$ (flow) & 13.9 & 11 & 1 & 1434 & 3059 & GI1_009026_NGC1518 \\
\hline 75 & NGC1518 & 040649.6 & -211029 & -45.31 & 10.8 & $\mathrm{v}$ (flow) & 12.3 & 8 & 0 & 1652 & 1737 & GI1_047024_NGC1518 \\
\hline 76 & ESO483-G013 & 041241.3 & -230936 & -44.58 & 10.4 & $\mathrm{v}$ (flow) & 14.2 & -3 & 1 & 2307 & 2307 & GI4_095007_ESO483_G013 \\
\hline 77 & NGC1556 & 041744.5 & -500952 & -44.78 & 10.5 & $\mathrm{v}$ (flow) & 13.5 & 2 & 0 & 1504 & 3101 & GI1_009038_NGC1556 \\
\hline 78 & UGCA90 & 042113.5 & -215044 & -42.31 & 10.4 & $\mathrm{v}$ (flow) & 12.7 & 7 & 0 & 1690 & 1690 & GI1_047026_UGCA090 \\
\hline 79 & NGC1592 & 042940.8 & -272431 & -41.91 & 10.6 & $\mathrm{v}$ (flow) & 14.6 & 10 & 0 & 1552 & 3248 & GI1_009020_HPJ0429m27 \\
\hline 80 & NGC1569 & 043049.0 & 645053 & 11.24 & 3.36 & trgb & 11.86 & 10 & 0 & 7148 & 7148 & NGA_NGC1569 \\
\hline 81 & UGCA92 & 043204.9 & 633649 & 10.52 & 1.8 & bs & 15.22 & 10 & 0 & 221 & 221 & AISCHV4_050_07132 \\
\hline 82 & NGC1560 & 043247.7 & 715246 & 16.02 & 3.45 & $\operatorname{trgb}$ & 12.2 & 7 & 0 & 209 & 209 & AISCHV4_050_04972 \\
\hline 83 & ESO158-G003 & 044616.7 & -572035 & -39.30 & 10.0 & $\mathrm{v}$ (flow) & 14.01 & 9 & 1 & 2654 & 2654 & GI4_095038_ESO158_G003 \\
\hline 84 & UGC3174 & 044834.5 & 001430 & -26.91 & 8.1 & $\mathrm{v}$ (flow) & 15.02 & 10 & 0 & 192 & 192 & AISCHV4_188_26696 \\
\hline 85 & ESO119-G016 & 045129.2 & -613903 & -37.77 & 9.8 & $\mathrm{v}$ (flow) & 14.8 & 10 & 1 & 3638 & 3638 & GI4_095008_ESO119_G016 \\
\hline 86 & NGC1705 & 045413.7 & -532141 & -38.74 & 5.10 & $\operatorname{trgb}$ & 12.8 & 11 & 1 & 1436 & 3673 & NGA_NGC1705 \\
\hline 87 & ESO252-IG001 & 045658.7 & -424814 & -38.50 & 6.0 & $\mathrm{v}$ (flow) & 14.4 & 99 & 0 & $\ldots$ & & \\
\hline 88 & NGC1744 & 045957.6 & -260119 & -35.02 & 7.7 & $\mathrm{v}$ (flow) & 11.6 & 6 & 1 & 1883 & 3874 & GI1_047027_NGC1744 \\
\hline 89 & NGC1796 & 050242.8 & -610823 & -36.55 & 10.3 & $\mathrm{v}$ (flow) & 12.9 & 5 & 1 & 2587 & 2587 & GI4_095009_NGC1796 \\
\hline 90 & ESO486-G021 & 050319.7 & -252523 & -34.13 & 8.9 & $\mathrm{v}$ (flow) & 14.5 & 2 & 1 & 1687 & 1687 & GI4_095010_ESO486_G021 \\
\hline 91 & MCG-05-13-004 & 050624.1 & -315711 & -35.12 & 6.6 & $\mathrm{v}$ (flow) & 13.2 & 9 & 1 & 1695 & 4598 & NGA_NGC1800 \\
\hline 92 & NGC1800 & 050625.4 & -315715 & -35.12 & 8.2 & $\mathrm{v}$ (flow) & 13.07 & 9 & 1 & 1695 & 4598 & NGA_NGC1800 \\
\hline 93 & NGC1808 & 050742.3 & -373046 & -35.90 & 10.6 & $\mathrm{v}$ (flow) & 10.76 & 1 & 0 & 2155 & 2155 & GI2_121004_LGG127_POS2 \\
\hline 94 & ESO305-G009 & 050807.6 & -381833 & -35.94 & 10.9 & $\mathrm{v}$ (flow) & 13 & 8 & 0 & 2829 & 2829 & GI1_009064_HPJ0508m38 \\
\hline 95 & UGCA103 & 051047.0 & -313550 & -34.14 & 10.4 & $\mathrm{v}$ (flow) & 13.1 & 9 & 0 & $\ldots$ & $\ldots$ & $\ldots$ \\
\hline 96 & UGCA106 & 051159.3 & -325821 & -34.20 & 9.8 & $\mathrm{v}$ (flow) & 13.1 & 9 & 1 & 1616 & 4439 & GI1_047029_UGCA106 \\
\hline 97 & UGCA105 & 051415.0 & 623431 & 13.66 & 3.15 & $\operatorname{trgb}$ & 14.5 & 10 & 0 & 216 & 216 & AISCHV4_095_02872 \\
\hline 98 & LMC & 052334.5 & -694522 & -32.89 & 0.05 & ceph & 0.91 & 9 & 1 & $\ldots$ & $\ldots$ & $\ldots$ \\
\hline 99 & UGC3303 & 052459.5 & 043018 & -16.92 & 7.2 & bs & 13.95 & 10 & 0 & $\ldots$ & $\ldots$ & $\ldots$ \\
\hline 100 & ESO553-G046 & 052705.7 & -204041 & -27.42 & 5.0 & $\mathrm{v}$ (flow) & 14.5 & 1 & 0 & 225 & 225 & AISCHV4_299_27736 \\
\hline 101 & ESO306-G013 & 053858.3 & -414414 & -30.67 & 10.8 & $\mathrm{v}$ (flow) & 14.2 & 3 & 0 & $\ldots$ & $\ldots$ & $\ldots$ \\
\hline 102 & UGCA114 & 055054.3 & -144645 & -19.94 & 9.8 & $\mathrm{v}$ (flow) & 12.97 & 7 & 0 & 110 & 110 & AISCHV4_296_27462 \\
\hline 103 & UGCA116 & 055542.6 & 032330 & -10.77 & 9.1 & $\mathrm{v}$ (flow) & 15.5 & 11 & 0 & 2465 & 2465 & GI3_089007_IIZw40 \\
\hline 104 & KKH34 & 055941.2 & 732539 & 22.35 & 4.61 & $\operatorname{trgb}$ & 17.10 & 10 & 0 & 214 & 214 & AISCHV3_094_02649 \\
\hline 105 & ESO364-G?029 & 060545.2 & -330451 & -23.37 & 7.4 & $\mathrm{v}$ (flow) & 14.0 & 10 & 0 & $\ldots$ & $\ldots$ & $\ldots$ \\
\hline 106 & AM0605-341 & 060719.3 & -341217 & -23.40 & 7.0 & $\mathrm{v}$ (flow) & 15.5 & 10 & 0 & $\ldots$ & $\ldots$ & $\ldots$ \\
\hline 107 & NGC2188 & 061009.5 & -340622 & -22.81 & 6.8 & $\mathrm{v}$ (flow) & 12.1 & 9 & 0 & $\ldots$ & $\ldots$ & $\ldots$ \\
\hline 108 & UGCA120 & 061116.3 & -213557 & -18.19 & 8.7 & $\mathrm{v}$ (flow) & 14.1 & 10 & 0 & $\ldots$ & $\ldots$ & $\ldots$ \\
\hline 109 & UGCA127 & 062055.5 & -82942 & -10.61 & 7.5 & $\mathrm{v}$ (flow) & 14.16 & 6 & 0 & $\ldots$ & $\ldots$ & $\ldots$ \\
\hline 110 & UGC3475 & 063028.8 & 393014 & 13.14 & 7.0 & $\mathrm{v}$ (flow) & 15.0 & 9 & 0 & $\ldots$ & $\ldots$ & $\ldots$ \\
\hline 111 & ESO255-G019 & 064548.2 & -473152 & -20.56 & 10.4 & $\mathrm{v}$ (flow) & 14 & 9 & 0 & $\ldots$ & $\ldots$ & $\ldots$ \\
\hline 112 & KKH37 & 064745.8 & 800726 & 26.54 & 3.26 & $\operatorname{trgb}$ & 16.40 & 10 & 1 & 1672 & 1672 & GI3_061011_kkh037 \\
\hline 113 & ESO207-G007 & 065039.9 & -520825 & -21.21 & 10.7 & $\mathrm{v}$ (flow) & 14.2 & 9 & 0 & 116 & 116 & AISCHV4_410_40316 \\
\hline 114 & UGC3600 & 065540.0 & 390543 & 17.48 & 7.3 & bs & 16.19 & 10 & 0 & 221 & 221 & AISCHV4_082_04070 \\
\hline 115 & AM0704-582 & 070518.8 & -583113 & -21.15 & 4.90 & $\operatorname{trgb}$ & 14.95 & 9 & 0 & $\ldots$ & $\ldots$ & $\ldots$ \\
\hline 116 & NGC2337 & 071013.5 & 442726 & 21.80 & 7.9 & bs & 13.48 & 10 & 0 & 220 & 220 & AISCHV4_078_03629 \\
\hline 117 & UGC3817 & 072244.5 & 450631 & 24.11 & 8.6 & bs & 15.96 & 10 & 0 & 87 & 87 & AISCHV4_078_03729 \\
\hline 118 & UGC3860 & 072817.2 & 404612 & 23.93 & 7.81 & $\operatorname{trgb}$ & 15.1 & 10 & 0 & 1688 & 1688 & GI4_016002_DDO43 \\
\hline 119 & NGC2366 & 072854.6 & 691257 & 28.53 & 3.21 & $\operatorname{trgb}$ & 11.43 & 10 & 1 & 2935 & 2935 & NGA_NGC2366 \\
\hline 120 & UGC3876 & 072917.5 & 275358 & 20.04 & 10.8 & $\mathrm{v}$ (flow) & 14.1 & 6 & 0 & $\ldots$ & $\ldots$ & $\ldots$ \\
\hline 121 & ESO059-G001 & 073118.2 & -681117 & -21.48 & 4.57 & $\operatorname{trgb}$ & 13.98 & 10 & 0 & $\ldots$ & $\ldots$ & . \\
\hline 122 & NGC2427 & 073627.9 & -473805 & -12.70 & 9.0 & $\mathrm{v}$ (flow) & 12.33 & 8 & 0 & $\ldots$ & $\ldots$ & $\ldots$ \\
\hline 123 & NGC2403 & 073651.4 & 653609 & 29.18 & 3.22 & ceph & 8.93 & 6 & 1 & 3317 & 3317 & NGA_NGC2403 \\
\hline 124 & UGC3966 & 074126.0 & 400644 & 26.17 & 6.8 & bs & 13.9 & 10 & 0 & 2857 & 2857 & GI4_016003_DDO46 \\
\hline 125 & UGC3974 & 074155.4 & 164809 & 18.54 & 8.04 & $\operatorname{trgb}$ & 13.6 & 10 & 0 & 2493 & 2493 & GI4_016004_DDO47 \\
\hline 126 & CGCG262-028 & 074732.1 & 511129 & 29.37 & 6.9 & $\mathrm{v}$ (flow) & 14.9 & 5 & 0 & 140 & 140 & AISCHV3_079_03433 \\
\hline 127 & UGC4115 & 075701.8 & 142327 & 20.90 & 7.72 & $\operatorname{trgb}$ & 15.23 & 10 & 0 & 1315 & 1315 & [4_042006_AOHI075702p142 \\
\hline
\end{tabular}


Table 1

(Continued)

\begin{tabular}{|c|c|c|c|c|c|c|c|c|c|c|c|c|}
\hline No. & $\begin{array}{c}\text { Galaxy Name } \\
\text { (2) }\end{array}$ & $\begin{array}{c}\text { R.A. } \\
\text { (J2000) } \\
(3)\end{array}$ & $\begin{array}{l}\text { Decl. } \\
(\mathrm{J} 2000) \\
(4)\end{array}$ & $\begin{array}{c}b \\
(\operatorname{deg}) \\
(5) \\
\end{array}$ & $\begin{array}{c}\mathrm{D} \\
(\mathrm{Mpc}) \\
(6)\end{array}$ & $\begin{array}{l}\text { Method } \\
\text { (7) }\end{array}$ & $\begin{array}{c}B \\
(\mathrm{mag}) \\
(8)\end{array}$ & $(9)$ & $\begin{array}{l}\text { Local Volume Legacy } \\
\qquad(10)\end{array}$ & $\begin{array}{l}t_{\text {fuv }} \\
(\mathrm{s}) \\
(11)\end{array}$ & $\begin{array}{l}t_{\text {nuv }} \\
(\mathrm{s}) \\
(12) \\
\end{array}$ & $\begin{array}{l}\text { Tile Name } \\
\text { (13) }\end{array}$ \\
\hline 128 & NGC2500 & 080153.3 & 504415 & 31.56 & 7.6 & $\mathrm{v}$ (flow) & 12.2 & 7 & 1 & 2979 & 2979 & NGA_NGC2500 \\
\hline 129 & NGC2537 & 081314.7 & 455926 & 32.96 & 6.9 & bs & 12.82 & 8 & 1 & 3204 & 3204 & NGA_NGC2537 \\
\hline 130 & UGC4278 & 081358.9 & 454434 & 33.06 & 7.6 & $\mathrm{v}$ (flow) & 13.1 & 7 & 1 & 3204 & 3204 & NGA_NGC2537 \\
\hline 131 & UGC4305 & 081904.0 & 704309 & 32.69 & 3.38 & $\operatorname{trgb}$ & 11.1 & 10 & 1 & 1677 & 1677 & NGA_HolmbergII \\
\hline 132 & NGC2552 & 081920.1 & 500025 & 34.29 & 7.7 & $\mathrm{v}$ (flow) & 12.6 & 9 & 1 & 1696 & 3386 & MISDR1_03473_0440 \\
\hline 133 & M81dwA & 082356.0 & 710145 & 33.01 & 3.44 & $\operatorname{trgb}$ & 18.69 & 10 & 1 & 1677 & 1677 & NGA_HolmbergII \\
\hline 134 & SDSSJ0825+3532 & 082555.5 & 353232 & 33.73 & 9.3 & $\mathrm{v}$ (flow) & 18.05 & 11 & 0 & 1120 & 2493 & NGA_HS0822p3542 \\
\hline 135 & UGC4426 & 082828.4 & 415124 & 35.21 & 10.28 & $\operatorname{trgb}$ & 15.0 & 10 & 1 & 1696 & 1696 & GI1_047032_UGC04426 \\
\hline 136 & UGC4459 & 083407.2 & 661054 & 34.95 & 3.61 & $\operatorname{trgb}$ & 14.78 & 10 & 1 & 1693 & 1693 & NGA_DDO053 \\
\hline 137 & ESO495-G021 & 083615.4 & -262434 & 8.58 & 7.8 & $\mathrm{v}$ (flow) & 12.46 & 11 & 0 & $\ldots$ & $\ldots$ & \\
\hline 138 & UGC4483 & 083703.0 & 694631 & 34.38 & 3.41 & $\operatorname{trgb}$ & 15.27 & 10 & 1 & 1393 & 1393 & NGA_UGC4483 \\
\hline 139 & NGC2683 & 085241.4 & 332514 & 38.76 & 7.7 & sbf & 10.64 & 3 & 1 & 1597 & 1597 & GI4_095011_NGC2683 \\
\hline 140 & UGC4704 & 085900.3 & 391236 & 40.74 & 7.8 & $\mathrm{v}$ (flow) & 15.3 & 8 & 1 & 1247 & 2287 & NGA_IRAS08572 \\
\hline 141 & LSBCD564-08 & 090253.8 & 200432 & 37.60 & 8.67 & $\operatorname{trgb}$ & 16.90 & 10 & 0 & 1582 & 1582 & GI4_016008_F564mV3 \\
\hline 142 & UGC4787 & 090734.9 & 331636 & 41.81 & 6.5 & $\mathrm{v}$ (flow) & 15.4 & 8 & 1 & 1595 & 1595 & GI4_095012_UGC04787 \\
\hline 143 & LSBCD634-03 & 090853.5 & 143455 & 36.94 & 9.46 & $\operatorname{trgb}$ & 17.50 & 10 & 0 & 149 & 149 & AISCHV3_194_23888 \\
\hline 144 & UGCA148 & 090946.5 & -230033 & 16.66 & 9.8 & mem & 15.3 & 10 & 0 & $\ldots$ & $\ldots$ & \\
\hline 145 & NGC2784 & 091219.5 & -241021 & 16.35 & 9.8 & $\operatorname{sbf}$ & 11.3 & -2 & 0 & 5861 & 7250 & NGA_NGC2784 \\
\hline 146 & UGCA153 & 091312.1 & -192431 & 19.59 & 6.5 & $\mathrm{v}$ (flow) & 15.40 & 10 & 0 & 116 & 116 & AISCHV4_210_25319 \\
\hline 147 & NGC2835 & 091752.9 & -222118 & 18.51 & 8.0 & $\mathrm{v}$ (flow) & 11.0 & 5 & 0 & 106 & 106 & AISCHV4_322_25486 \\
\hline 148 & LSBCD565-06 & 091930.0 & 213612 & 41.76 & 9.08 & $\operatorname{trgb}$ & 16.95 & 10 & 0 & 112 & 112 & AISCHV3_193_16500 \\
\hline 149 & UGCA162 & 092128.1 & -223007 & 19.03 & 7.5 & $\mathrm{v}$ (flow) & 15.2 & 9 & 0 & 117 & 117 & AISCHV4_322_25486 \\
\hline 150 & UGC4998 & 092512.1 & 682259 & 38.89 & 10.5 & $\mathrm{sbf}$ & 14.72 & 10 & 1 & 1948 & 1948 & NGA_Arp300 \\
\hline 151 & NGC2915 & 092611.5 & -763736 & -18.36 & 3.78 & $\operatorname{trgb}$ & 13.20 & 0 & 0 & 4728 & 4728 & NGA_NGC2915 \\
\hline 152 & NGC2903 & 093210.1 & 213004 & 44.54 & 8.9 & bs & 9.68 & 4 & 1 & 1915 & 1915 & NGA_NGC2903 \\
\hline 153 & UGC5076 & 093236.4 & 515219 & 45.57 & 8.3 & $\mathrm{v}$ (flow) & 15.2 & 10 & 1 & $\ldots$ & $\ldots$ & $\cdots$ \\
\hline 154 & CGCG035-007 & 093444.9 & 062532 & 38.99 & 5.2 & $\mathrm{v}$ (flow) & 15.5 & 5 & 1 & 1869 & 3472 & GI1_047033_CGCG035_007 \\
\hline 155 & LeoT & 093453.4 & 170305 & 43.66 & 0.42 & $\operatorname{trgb}$ & 16.40 & 10 & 0 & 1549 & 1550 & NGA_LeoT \\
\hline 156 & UGC5151 & 094027.1 & 482015 & 47.60 & 10.7 & $\mathrm{v}$ (flow) & 13.8 & 10 & 0 & $\ldots$ & $\ldots$ & $\ldots$ \\
\hline 157 & UGC5139 & 094032.3 & 711056 & 38.66 & 3.90 & $\operatorname{trgb}$ & 14.17 & 10 & 1 & 1704 & 1704 & NGA_HolmbergI \\
\hline 158 & IC559 & 094443.9 & 093655 & 42.70 & 4.9 & $\mathrm{v}$ (flow) & 14.8 & 5 & 1 & 1470 & 2704 & GI1_047034_IC0559 \\
\hline 159 & UGC5209 & 094504.2 & 321418 & 49.51 & 6.4 & $\mathrm{v}$ (flow) & 16.06 & 10 & 0 & 208 & 208 & AISCHV4_214_02210 \\
\hline 160 & NGC2976 & 094715.3 & 675500 & 40.90 & 3.56 & $\operatorname{trgb}$ & 11.24 & 5 & 1 & 1693 & 1693 & GI3_061016_KK77 \\
\hline 161 & UGC5272b & 095019.4 & 312721 & 50.55 & 7.1 & mem & 17.76 & 10 & 0 & 1542 & 1543 & GI4_095013_UGC05272 \\
\hline 162 & UGC5272 & 095022.4 & 312916 & 50.56 & 7.1 & bs & 15.41 & 10 & 1 & 1542 & 1543 & GI4_095013_UGC05272 \\
\hline 163 & UGC5288 & 095117.0 & 074939 & 43.25 & 6.8 & bs & 14.32 & 8 & 1 & 1676 & 1676 & GI1_047035_UGC05288 \\
\hline 164 & NGC3037 & 095123.5 & -270036 & 20.70 & 7.9 & $\mathrm{v}$ (flow) & 13.7 & 9 & 0 & 218 & 218 & AISCHV4_322_25658 \\
\hline 165 & BK3N & 095348.5 & 685809 & 40.83 & 3.86 & $\operatorname{trgb}$ & 18.78 & 10 & 1 & 3075 & 3075 & NGA_M81andM82 \\
\hline 166 & NGC3031 & 095533.2 & 690355 & 40.90 & 3.63 & ceph & 7.89 & 2 & 1 & 3075 & 3075 & NGA_M81andM82 \\
\hline 167 & NGC3034 & 095552.2 & 694047 & 40.57 & 3.37 & $\operatorname{trgb}$ & 9.30 & 7 & 1 & 3075 & 3075 & NGA_M81andM82 \\
\hline 168 & UGC5340 & 095645.7 & 284935 & 51.62 & 5.9 & bs & 14.8 & 10 & 1 & 1439 & 1439 & GI1_047036_UGC05340 \\
\hline 169 & KDG61 & 095703.1 & 683531 & 41.28 & 3.49 & $\operatorname{trgb}$ & 15.17 & -6 & 1 & 16218 & 16218 & GI2_024001_NGC3077_Stream \\
\hline 170 & UGC5336 & 095732.0 & 690245 & 41.06 & 3.61 & $\operatorname{trgb}$ & 14.3 & 10 & 1 & 3075 & 3075 & NGA_M81andM82 \\
\hline 171 & ESO435-G016 & 095846.2 & -283719 & 20.57 & 9.1 & $\mathrm{v}$ (flow) & 13.6 & 3 & 0 & 4228 & 5882 & NGA_Tol2 \\
\hline 172 & ESO435-IG020 & 095920.7 & -280754 & 21.03 & 9.0 & $\mathrm{v}$ (flow) & 14.4 & 10 & 0 & 4228 & 5882 & NGA_Tol2 \\
\hline 173 & UGC5364 & 095926.4 & 304447 & 52.42 & 0.69 & $\operatorname{trgb}$ & 12.9 & 10 & 1 & 1536 & 1536 & GI4_016005_DDO69 \\
\hline 174 & UGC5373 & 100000.1 & 051956 & 43.78 & 1.39 & $\operatorname{trgb}$ & 11.8 & 10 & 1 & 1085 & 1085 & NGA_SextansB \\
\hline 175 & UGCA193 & 100236.0 & -60049 & 37.43 & 9.7 & mem & 14.84 & 7 & 1 & 2169 & 2169 & GI4_095015_UGCA193 \\
\hline 176 & NGC3109 & 100306.6 & -260932 & 23.07 & 1.28 & $\operatorname{trgb}$ & 10.39 & 9 & 1 & 10338 & 13636 & NGA_NGC3109 \\
\hline 177 & NGC3077 & 100320.6 & 684404 & 41.66 & 3.83 & $\operatorname{trgb}$ & 10.6 & 6 & 1 & $\ldots$ & $\ldots$ & $\ldots$ \\
\hline 178 & UGCA196 & 100341.8 & -270140 & 22.49 & 8.8 & $\mathrm{v}$ (flow) & 13.4 & 6 & 0 & 11735 & 15032 & NGA_Antlia_Dw \\
\hline 179 & NGC3113 & 100426.1 & -282639 & 21.51 & 10.5 & $\mathrm{v}$ (flow) & 13.3 & 7 & 0 & $\ldots$ & $\ldots$ & \\
\hline 180 & UGC5427 & 100441.0 & 292155 & 53.40 & 7.1 & bs & 15.9 & 8 & 0 & 1594 & 1594 & GI1_047038_UGC05427 \\
\hline 181 & UGC5428 & 100506.4 & 663332 & 43.09 & 3.53 & $\operatorname{trgb}$ & 15.95 & 10 & 1 & 1704 & 1704 & GI3_061017_UGC05428 \\
\hline 182 & NGC3115 & 100514.0 & -74307 & 36.78 & 9.7 & $\operatorname{sbf}$ & 9.87 & -3 & 0 & 1304 & 1304 & NGA_NGC3115 \\
\hline 183 & UGC5423 & 100530.6 & 702152 & 40.81 & 5.3 & bs & 15.20 & 10 & 1 & 1698 & 1698 & NGA_M81DwB \\
\hline 184 & NGC3125 & 100633.6 & -295609 & 20.64 & 10.8 & $\mathrm{v}$ (flow) & 13.5 & 11 & 0 & 3024 & 5927 & NGA_NGC3125 \\
\hline 185 & UGC5453 & 100707.3 & 155904 & 50.40 & 9.3 & $\mathrm{v}$ (flow) & 15.8 & 10 & 0 & $\ldots$ & $\ldots$ & $\ldots$ \\
\hline 186 & UGC5451 & 100719.0 & 470022 & 52.31 & 8.7 & $\mathrm{v}$ (flow) & 14.4 & 10 & 0 & 122 & 122 & AISCHV3_081_01122 \\
\hline 187 & UGC5456 & 100719.6 & 102146 & 47.94 & 3.80 & $\operatorname{trgb}$ & 13.7 & 5 & 1 & 1552 & 1552 & GI4_095016_UGC05456 \\
\hline 188 & KUG1004+392 & 100723.0 & 385810 & 53.99 & 7.8 & $\mathrm{v}$ (flow) & 16.0 & 10 & 0 & 119 & 119 & AISCHV3_086_01586 \\
\hline 189 & NGC3137 & 100907.5 & -290352 & 21.67 & 10.8 & $\mathrm{v}$ (flow) & 12.1 & 6 & 0 & $\ldots$ & $\ldots$ & $\ldots$ \\
\hline 190 & SextansA & 101100.8 & -44134 & 39.88 & 1.32 & ceph & 11.86 & 10 & 1 & 1689 & 1689 & NGA_SextansA \\
\hline 191 & NGC3175 & 101442.1 & -285219 & 22.58 & 10.7 & $\mathrm{v}$ (flow) & 12.13 & 2 & 0 & 225 & 225 & AISCHV4_328_25973 \\
\hline
\end{tabular}


Table 1

(Continued)

\begin{tabular}{|c|c|c|c|c|c|c|c|c|c|c|c|c|}
\hline No. & Galaxy Name & $\begin{array}{c}\text { R.A. } \\
(\mathrm{J} 2000) \\
(3)\end{array}$ & $\begin{array}{c}\text { Decl. } \\
(\mathrm{J} 2000) \\
(4)\end{array}$ & $\begin{array}{c}b \\
(\mathrm{deg}) \\
(5)\end{array}$ & $\begin{array}{c}\mathrm{D} \\
(\mathrm{Mpc}) \\
(6)\end{array}$ & Method & $\begin{array}{c}B \\
(\mathrm{mag}) \\
(8)\end{array}$ & (9) & Local Volume Legacy & $\begin{array}{c}t_{\text {fuv }} \\
(\mathrm{s}) \\
(11)\end{array}$ & $\begin{array}{c}t_{\text {nuv }} \\
(\mathrm{s}) \\
(12)\end{array}$ & Tile Name \\
\hline 192 & NGC3239 & 102505.6 & 170937 & 54.82 & 8.3 & $\mathrm{v}$ (flow) & 11.7 & 9 & 1 & 1766 & 1766 & GI1_047039_NGC3239 \\
\hline 193 & UGC5672 & 102820.9 & 223417 & 57.33 & 6.3 & bs & 15.1 & 5 & 1 & 1772 & 1772 & GI1_047040_UGC05672 \\
\hline 194 & UGC5666 & 102821.2 & 682443 & 43.61 & 3.81 & $\operatorname{trgb}$ & 10.8 & 9 & 1 & 1848 & 3520 & NGA_IC2574 \\
\hline 195 & UGC5692 & 103035.0 & 703707 & 42.18 & 3.80 & $\operatorname{trgb}$ & 13.71 & 9 & 1 & 1821 & 3505 & GI1_047041_UGC05692 \\
\hline 196 & NGC3274 & 103217.1 & 274007 & 59.21 & 6.5 & bs & 13.2 & 7 & 1 & 1661 & 1661 & GI1_047042_NGC3274 \\
\hline 197 & UGC5740 & 103445.8 & 504606 & 54.98 & 9.3 & $\mathrm{v}$ (flow) & 15.6 & 9 & 0 & 100 & 100 & AISCHV4_092_01025 \\
\hline 198 & UGCA212 & 103523.2 & -244515 & 28.62 & 10.1 & $\mathrm{v}$ (flow) & 13.2 & 8 & 0 & 175 & 175 & AISCHV4_324_25706 \\
\hline 199 & NGC3299 & 103623.8 & 124227 & 55.29 & 10.4 & mem & 14.1 & 8 & 1 & 1076 & 2253 & GI4_095017_NGC3299 \\
\hline 200 & UGC5764 & 103643.3 & 313248 & 60.43 & 7.1 & $\mathrm{v}$ (flow) & 15.2 & 10 & 1 & 1614 & 1614 & GI1_047043_UGC05764 \\
\hline 201 & UGC5797 & 103925.2 & 014305 & 49.44 & 6.8 & $\mathrm{v}$ (flow) & 15.0 & 10 & 1 & 1660 & 1660 & GI1_047044_UGC05797 \\
\hline 202 & IC625 & 104238.0 & -235608 & 30.22 & 10.1 & $\mathrm{v}$ (flow) & 13.8 & 5 & 0 & 214 & 214 & AISCHV4_324_25582 \\
\hline 203 & UGC5829 & 104242.2 & 342656 & 61.53 & 7.9 & $\mathrm{v}$ (flow) & 13.7 & 10 & 1 & 2189 & 2189 & GI1_047045_UGC05829 \\
\hline 204 & NGC3344 & 104330.9 & 245522 & 61.26 & 6.6 & $\mathrm{v}$ (flow) & 10.4 & 4 & 1 & 1414 & 1718 & NGA_NGC3344 \\
\hline 205 & NGC3351 & 104357.8 & 114214 & 56.37 & 10.00 & ceph & 10.53 & 3 & 1 & 1701 & 1701 & NGA_NGC3351 \\
\hline 206 & NGC3365 & 104612.6 & 014848 & 50.75 & 10.5 & $\mathrm{v}$ (flow) & 13.2 & 6 & 0 & 2987 & 2987 & GI1_047046_NGC3365 \\
\hline 207 & NGC3368 & 104645.7 & 114912 & 57.01 & 10.52 & ceph & 10.1 & 2 & 1 & 1698 & 1698 & NGA_NGC3368 \\
\hline 208 & UGC5889 & 104722.3 & 140410 & 58.29 & 9.3 & bs & 14.2 & 9 & 1 & 1639 & 1639 & GI3_084014_J104728p135322 \\
\hline 209 & UGC5917 & 104853.9 & 464314 & 58.98 & 10.3 & $\mathrm{v}$ (flow) & 16.1 & 10 & 0 & 106 & 106 & AISCHV4_091_01363 \\
\hline 210 & UGC5923 & 104907.6 & 065502 & 54.64 & 7.2 & $\mathrm{v}($ flow $)$ & 14.0 & 0 & 1 & 3259 & 3259 & GI3_046013_UGC5923 \\
\hline 211 & UGC5918 & 104936.5 & 653150 & 47.12 & 7.4 & bs & 15.22 & 10 & 1 & 1530 & 1530 & GI4_016006_DDO87 \\
\hline 212 & NGC3412 & 105053.3 & 132444 & 58.70 & 10.4 & mem & 11.4 & -2 & 0 & 1641 & 1641 & GI3_084016_J104952p130942 \\
\hline 213 & ESO376-G022 & 105121.0 & -342550 & 22.20 & 9.0 & $\mathrm{v}$ (flow) & 14.2 & 10 & 0 & $\ldots$ & $\ldots$ & $\ldots$ \\
\hline 214 & NGC3432 & 105231.3 & 363711 & 63.16 & 7.9 & $\mathrm{v}$ (flow) & 11.7 & 9 & 1 & 1717 & 1717 & GI4_095018_NGC3432 \\
\hline 215 & KDG73 & 105257.1 & 693258 & 44.23 & 4.03 & $\operatorname{trgb}$ & 17.28 & 10 & 1 & 1703 & 1703 & GI1_047048_KDG073 \\
\hline 216 & NGC3489 & 110018.6 & 135404 & 60.91 & 10.4 & mem & 11.1 & -1 & 0 & 1642 & 1642 & GI3_087007_NGC3489 \\
\hline 217 & NGC3486 & 110023.9 & 285830 & 65.49 & 8.2 & $\mathrm{v}$ (flow) & 11.05 & 5 & 1 & 1488 & 3069 & GI4_095046_NGC3486 \\
\hline 218 & UGC6102 & 110148.4 & 284121 & 65.78 & 8.5 & $\mathrm{v}$ (flow) & 15.5 & 10 & 0 & 541 & 1989 & NGA_NGC3486 \\
\hline 219 & NGC3510 & 110343.4 & 285313 & 66.21 & 8.6 & $\mathrm{v}$ (flow) & 14 & 8 & 1 & 1565 & 1565 & GI1_047049_NGC3510 \\
\hline 220 & ESO377-G003 & 110355.2 & -342130 & 23.45 & 9.2 & $\mathrm{v}$ (flow) & 14.9 & 4 & 0 & 110 & 110 & AISCHV4_455_37081 \\
\hline 221 & MRK36 & 110458.5 & 290822 & 66.49 & 7.8 & $\mathrm{v}$ (flow) & 15.7 & 11 & 0 & 1565 & 1565 & GI1_047049_NGC3510 \\
\hline 222 & NGC3521 & 110548.6 & -00209 & 52.83 & 8.0 & $\mathrm{v}$ (flow) & 9.83 & 4 & 1 & 1809 & 1809 & NGA_NGC3521 \\
\hline 223 & UGC6161 & 110649.2 & 434324 & 63.19 & 10.3 & $\mathrm{v}$ (flow) & 15 & 8 & 0 & 1460 & 1460 & GI3_121012_J110638p432344 \\
\hline 224 & MESSIER108 & 111131.0 & 554027 & 56.25 & 10.4 & $\mathrm{v}$ (flow) & 10.69 & 6 & 0 & 1623 & 1623 & GI4_034001_M108 \\
\hline 225 & NGC3593 & 111437.0 & 124904 & 63.21 & 6.5 & $\mathrm{v}$ (flow) & 11.86 & 0 & 1 & 1596 & 1596 & GI4_095041_NGC3593 \\
\hline 226 & NGC3599 & 111527.0 & 180637 & 66.14 & 9.6 & $\mathrm{v}$ (flow) & 12.8 & -2 & 0 & 2468 & 2468 & GI3_079016_NGC3608 \\
\hline 227 & NGC3600 & 111552.1 & 413532 & 65.68 & 9.7 & $\mathrm{v}$ (flow) & 14.0 & 1 & 0 & 1583 & 1583 & GI1_047050_NGC3600 \\
\hline 228 & NGC3621 & 111816.0 & -324842 & 26.10 & 6.64 & ceph & 10.2 & 7 & 0 & 10926 & 10926 & NGA_NGC3621 \\
\hline 229 & NGC3623 & 111855.9 & 130537 & 64.22 & 8.9 & $\mathrm{v}$ (flow) & 10.25 & 1 & 1 & 1650 & 1650 & GI3_041004_NGC3623 \\
\hline 230 & NGC3627 & 112015.0 & 125930 & 64.42 & 10.05 & ceph & 9.7 & 3 & 1 & 1680 & 3072 & NGA_NGC3627 \\
\hline 231 & NGC3628 & 112016.9 & 133520 & 64.78 & 9.4 & $\mathrm{v}$ (flow) & 10.28 & 3 & 1 & 7511 & 7512 & NGA_NGC3628_cen \\
\hline 232 & IC2782 & 112255.3 & 132629 & 65.21 & 9.7 & $\mathrm{v}$ (flow) & 14.9 & 8 & 0 & 102 & 1625 & $\begin{array}{c}\text { AISCHV4_232_12480, } \\
\text { GI5_002014_HI1123377p125353_0001 }\end{array}$ \\
\hline 233 & IC2787 & 112319.1 & 133747 & 65.40 & 7.7 & $\mathrm{v}$ (flow) & 16.1 & 6 & 0 & $\ldots$ & $\ldots$ & $\ldots$ \\
\hline 234 & NGC3675 & 112608.6 & 433509 & 66.19 & 10.6 & $\mathrm{v}$ (flow) & 11.0 & 3 & 0 & 105 & 105 & AISCHV4_102_01355 \\
\hline 235 & UGC6457 & 112712.2 & -05941 & 55.34 & 10.2 & $\mathrm{v}$ (flow) & 15 & 10 & 1 & 2241 & 3353 & GI4_095019_UGC06457 \\
\hline 236 & UGC6456 & 112759.9 & 785939 & 37.33 & 4.34 & $\operatorname{trgb}$ & 15.9 & 10 & 0 & 1642 & 1642 & NGA_VIIZw403 \\
\hline 237 & UGC6541 & 113328.9 & 491414 & 63.28 & 3.89 & $\operatorname{trgb}$ & 14.40 & 11 & 1 & 1648 & 1648 & GI4_016011_MRK178 \\
\hline 238 & NGC3738 & 113548.8 & 543126 & 59.31 & 4.90 & $\operatorname{trgb}$ & 11.97 & 10 & 1 & 1620 & 1620 & GI4_016009_NGC3738 \\
\hline 239 & NGC3741 & 113606.2 & 451701 & 66.45 & 3.24 & $\operatorname{trgb}$ & 14.49 & 10 & 1 & 1663 & 3231 & GI1_047051_NGC3741 \\
\hline 240 & LEDA166115 & 114711.2 & 434019 & 68.98 & 4.51 & $\operatorname{trgb}$ & 18.62 & 10 & 0 & 122 & 122 & AISCHV3_102_01354 \\
\hline 241 & UGC6817 & 115053.0 & 385249 & 72.74 & 2.59 & $\operatorname{trgb}$ & 13.56 & 10 & 1 & 2373 & 2373 & GI1_047053_UGC06817 \\
\hline 242 & UGC6900 & 115539.4 & 313110 & 77.08 & 7.5 & $\mathrm{v}$ (flow) & 14.8 & 10 & 1 & 1677 & 1677 & GI1_047054_UGC06900 \\
\hline 243 & BTS76 & 115844.1 & 273506 & 78.29 & 6.0 & $\mathrm{v}$ (flow) & 16.50 & 10 & 0 & 1417 & 1417 & GI1_026016_Arp305 \\
\hline 244 & NGC4020 & 115856.6 & 302444 & 78.05 & 9.7 & $\mathrm{v}($ flow $)$ & 13.8 & 7 & 1 & 1680 & 1680 & GI1_047055_NGC4020 \\
\hline 245 & UGC7007 & 120133.1 & 332029 & 77.58 & 10.1 & $\mathrm{v}$ (flow) & 16.30 & 9 & 0 & 136 & 136 & AISCHV4_112_12396 \\
\hline 246 & NGC4068 & 120400.8 & 523518 & 63.04 & 4.31 & $\operatorname{trgb}$ & 13.02 & 10 & 1 & 2569 & 2569 & GI4_095022_NGC4068 \\
\hline 247 & NGC4080 & 120451.8 & 265933 & 79.63 & 6.9 & $\mathrm{v}$ (flow) & 14.3 & 10 & 1 & 1688 & 1688 & GI1_047056_NGC4080 \\
\hline 248 & NGC4096 & 120601.0 & 472840 & 67.79 & 8.3 & $\mathrm{v}$ (flow) & 11.48 & 5 & 1 & 1660 & 1660 & GI1_047058_NGC4096 \\
\hline 249 & KUG1207+367 & 120956.4 & 362607 & 77.20 & 4.5 & $\mathrm{v}$ (flow) & 15.50 & 10 & 0 & 7205 & 10324 & GI4_015004_NGC4163 \\
\hline 250 & NGC4144 & 120958.4 & 462727 & 69.01 & 9.8 & bs & 12.1 & 6 & 1 & 1664 & 4541 & GI1_047059_NGC4144 \\
\hline 251 & NGC4163 & 121209.1 & 361009 & 77.70 & 2.87 & $\operatorname{trgb}$ & 13.75 & 10 & 1 & 1677 & 1677 & GI3_061001_NGC4163 \\
\hline 252 & NGC4190 & 121344.7 & 363803 & 77.59 & 3.5 & bs & 13.9 & 10 & 1 & 1677 & 1677 & GI3_061001_NGC4163 \\
\hline 253 & UGC7242 & 121408.4 & 660541 & 50.60 & 5.42 & $\operatorname{trgb}$ & 14.7 & 6 & 1 & 1697 & 1697 & MISDR1_00419_0493 \\
\hline 254 & UGCA276 & 121457.9 & 361308 & 78.06 & 2.95 & $\operatorname{trgb}$ & 15.70 & 10 & 1 & 1677 & 1677 & GI3_061001_NGC4163 \\
\hline
\end{tabular}


Table 1

(Continued)

\begin{tabular}{|c|c|c|c|c|c|c|c|c|c|c|c|c|}
\hline No. & $\begin{array}{c}\text { Galaxy Name } \\
\text { (2) }\end{array}$ & $\begin{array}{c}\text { R.A. } \\
(J 2000) \\
(3)\end{array}$ & $\begin{array}{c}\text { Decl. } \\
(\mathrm{J} 2000) \\
(4)\end{array}$ & $\begin{array}{c}b \\
(\mathrm{deg}) \\
(5) \\
\end{array}$ & $\begin{array}{c}\mathrm{D} \\
(\mathrm{Mpc}) \\
(6)\end{array}$ & Method & $\begin{array}{c}B \\
(\mathrm{mag}) \\
(8)\end{array}$ & (9) & $\begin{array}{l}\text { Local Volume Legacy } \\
\text { (10) }\end{array}$ & $\begin{array}{c}t_{\text {fuv }} \\
(\mathrm{s}) \\
(11)\end{array}$ & $\begin{array}{c}t_{\text {nuv }} \\
(\mathrm{s}) \\
(12)\end{array}$ & $\begin{array}{c}\text { Tile Name } \\
\text { (13) }\end{array}$ \\
\hline 255 & NGC4204 & 121514.5 & 203931 & 79.50 & 10.4 & $\mathrm{v}$ (flow) & 14.0 & 8 & 0 & 1546 & 1693 & GI1_047061_NGC4204 \\
\hline 256 & UGC7267 & 121523.6 & 512058 & 64.84 & 7.3 & $\mathrm{v}$ (flow) & 15.3 & 8 & 1 & 1658 & 1658 & GI1_047062_UGC07267 \\
\hline 257 & UGC7271 & 121533.3 & 432606 & 72.15 & 7.8 & $\mathrm{v}$ (flow) & 15.53 & 7 & 0 & 1669 & 2568 & GI1_047063_UGC07271 \\
\hline 258 & NGC4214 & 121538.9 & 361940 & 78.07 & 3.04 & $\operatorname{trgb}$ & 10.2 & 10 & 1 & 2003 & 2003 & GI4_095023_NGC4214 \\
\hline 259 & CGCG269-049 & 121546.7 & 522315 & 63.87 & 3.2 & $\mathrm{v}$ (flow) & 15.30 & 10 & 1 & 1652 & 1652 & GI3_061013_CGCG269_049 \\
\hline 260 & UGC7298 & 121630.1 & 521339 & 64.06 & 4.21 & $\operatorname{trgb}$ & 16.06 & 10 & 0 & 1674 & 1674 & GI3_061013_CGCG269_049 \\
\hline 261 & NGC4236 & 121642.1 & 692746 & 47.36 & 4.45 & $\operatorname{trgb}$ & 10.1 & 8 & 1 & 1690 & 1690 & NGA_NGC4236 \\
\hline 262 & NGC4244 & 121729.9 & 374829 & 77.16 & 4.49 & $\operatorname{trgb}$ & 10.9 & 6 & 1 & 1607 & 3087 & GI1_047064_NGC4244 \\
\hline 263 & NGC4242 & 121730.1 & 453708 & 70.32 & 7.4 & $\mathrm{v}$ (flow) & 11.4 & 8 & 1 & 559 & 2250 & NGA_NGC4242 \\
\hline 264 & NGC4248 & 121750.3 & 472431 & 68.68 & 7.2 & $\mathrm{v}$ (flow) & 13.21 & 3 & 1 & 1642 & 1642 & GI4_095048_NGC4258 \\
\hline 265 & IC3104 & 121846.0 & -794334 & -16.95 & 2.27 & $\operatorname{trgb}$ & 13.5 & 10 & 0 & $\ldots$ & $\ldots$ & $\ldots$ \\
\hline 266 & NGC4258 & 121857.5 & 471814 & 68.84 & 7.98 & ceph & 9.10 & 4 & 1 & 1642 & 1642 & GI4_095048_NGC4258 \\
\hline 267 & UGC7356 & 121909.1 & 470523 & 69.05 & 6.7 & $\mathrm{sbf}$ & 15.58 & 10 & 0 & $\ldots$ & 1674 & $\ldots$ \\
\hline 268 & ISZ399 & 121959.5 & -172331 & 44.83 & 9.0 & $\mathrm{v}$ (flow) & 14.7 & 11 & 1 & $\ldots$ & $\ldots$ & $\ldots$ \\
\hline 269 & NGC4288 & 122038.1 & 461733 & 69.89 & 7.7 & $\mathrm{v}$ (flow) & 13.3 & 7 & 1 & 1510 & 2756 & GI1_047066_NGC4288 \\
\hline 270 & UGC7408 & 122115.0 & 454841 & 70.38 & 6.9 & $\mathrm{v}$ (flow) & 13.3 & 9 & 1 & 1510 & 2756 & GI1_047066_NGC4288 \\
\hline 271 & UGC7490 & 122425.3 & 702001 & 46.62 & 8.4 & $\mathrm{v}$ (flow) & 13.1 & 9 & 1 & $\ldots$ & $\ldots$ & $\ldots$ \\
\hline 272 & LEDA166137 & 122527.9 & 282857 & 84.10 & 6.0 & $\mathrm{v}$ (flow) & 16.50 & 10 & 0 & 95 & 95 & AISCHV4_113_12847 \\
\hline 273 & NGC4395 & 122548.9 & 333248 & 81.53 & 4.61 & $\operatorname{trgb}$ & 10.6 & 9 & 1 & 1696 & 1696 & NGA_NGC4395 \\
\hline 274 & UGCA281 & 122616.0 & 482937 & 68.08 & 5.7 & bs & 15.4 & 11 & 1 & 1672 & 1672 & GI1_047067_UGCA281 \\
\hline 275 & UGC7559 & 122705.1 & 370833 & 78.74 & 4.87 & $\operatorname{trgb}$ & 14.2 & 10 & 1 & 1680 & 1680 & GI1_047068_UGC07559 \\
\hline 276 & UGC7577 & 122740.9 & 432944 & 72.94 & 2.58 & $\operatorname{trgb}$ & 12.8 & 10 & 1 & 1687 & 1687 & GI1_047069_UGC07577 \\
\hline 277 & UGC7584 & 122802.9 & 223522 & 83.02 & 7.3 & $\mathrm{v}$ (flow) & 16.20 & 9 & 0 & 2462 & 2462 & GI1_047071_NGC4455 \\
\hline 278 & LSBCF573-01 & 122805.4 & 221727 & 82.82 & 7.2 & $\mathrm{v}$ (flow) & 17.00 & 10 & 0 & 2462 & 2462 & GI1_047071_NGC4455 \\
\hline 279 & NGC4449 & 122811.2 & 440536 & 72.40 & 4.21 & $\operatorname{trgb}$ & 10.0 & 10 & 1 & 845 & 845 & NGA_NGC4449 \\
\hline 280 & UGC7599 & 122828.5 & 371401 & 78.79 & 6.9 & bs & 14.88 & 8 & 1 & 1680 & 1680 & GI1_047068_UGC07559 \\
\hline 281 & UGC7605 & 122838.7 & 354303 & 80.14 & 4.43 & $\operatorname{trgb}$ & 14.8 & 10 & 1 & 1680 & 1680 & GI1_047070_UGC07605 \\
\hline 282 & NGC4455 & 122844.1 & 224921 & 83.29 & 7.8 & $\mathrm{v}$ (flow) & 14 & 7 & 1 & 2462 & 2462 & GI1_047071_NGC4455 \\
\hline 283 & UGC7608 & 122845.3 & 431335 & 73.26 & 7.8 & $\mathrm{v}$ (flow) & 13.7 & 10 & 1 & 1687 & 1687 & GI1_047069_UGC07577 \\
\hline 284 & NGC4460 & 122845.5 & 445151 & 71.69 & 9.6 & sbf & 12.8 & -1 & 1 & 845 & 845 & NGA_NGC4449 \\
\hline 285 & MCG+07-26-011 & 122852.2 & 421041 & 74.26 & 6.0 & $\mathrm{v}$ (flow) & 16.33 & 8 & 0 & 1587 & 2810 & NGA_NGC4490 \\
\hline 286 & UGC7639 & 122953.4 & 473152 & 69.17 & 8.0 & bs & 13.99 & 10 & 1 & 3249 & 5883 & GI1_047072_UGC07639 \\
\hline 287 & MCG+07-26-012 & 123023.8 & 425406 & 73.66 & 6.4 & $\mathrm{v}$ (flow) & 16.47 & 6 & 0 & 1671 & 1671 & GI4_095025_UGC7690 \\
\hline 288 & NGC4485 & 123031.1 & 414201 & 74.81 & 7.1 & $\mathrm{v}$ (flow) & 12.32 & 10 & 1 & 1587 & 2810 & NGA_NGC4490 \\
\hline 289 & NGC4490 & 123036.1 & 413834 & 74.87 & 8.0 & $\mathrm{v}$ (flow) & 10.22 & 7 & 1 & 1587 & 2810 & NGA_NGC4490 \\
\hline 290 & UGC7678 & 123200.7 & 394959 & 76.67 & 9.3 & $\mathrm{v}$ (flow) & 16 & 6 & 0 & 103 & 103 & AISCHV3_107_01912 \\
\hline 291 & UGC7690 & 123226.8 & 424218 & 73.95 & 7.7 & $\mathrm{v}$ (flow) & 13.1 & 10 & 1 & 1671 & 1671 & GI4_095025_UGC7690 \\
\hline 292 & UGC7699 & 123248.0 & 373718 & 78.80 & 6.8 & $\mathrm{v}$ (flow) & 13.6 & 6 & 1 & 1654 & 1654 & GI1_047074_UGC07699 \\
\hline 293 & UGC7698 & 123254.4 & 313228 & 84.02 & 6.1 & bs & 13.0 & 10 & 1 & 1671 & 1671 & GI1_047075_UGC07698 \\
\hline 294 & UGC7719 & 123400.6 & 390110 & 77.57 & 9.4 & $\mathrm{v}$ (flow) & 15.3 & 8 & 1 & 1677 & 3287 & GI1_047076_UGC07719 \\
\hline 295 & NGC4534 & 123405.4 & 353108 & 80.83 & 10.8 & $\mathrm{v}$ (flow) & 13.0 & 8 & 0 & 1677 & 1677 & GI1_047077_NGC4534 \\
\hline 296 & UGC7774 & 123622.5 & 400019 & 76.75 & 7.4 & $\mathrm{v}$ (flow) & 15.02 & 7 & 1 & 1661 & 1661 & GI4_095042_UGC7774 \\
\hline 297 & UGCA290 & 123721.8 & 384438 & 78.02 & 6.70 & $\operatorname{trgb}$ & 15.74 & 11 & 0 & 121 & 121 & AISCHV4_107_01563 \\
\hline 298 & UGCA292 & 123840.0 & 324601 & 83.72 & 3.62 & $\operatorname{trgb}$ & 16.10 & 10 & 1 & 1728 & 3301 & GI4_016001_CVNIDWA \\
\hline 299 & M104 & 123959.4 & -113723 & 51.15 & 9.3 & $\mathrm{sbf}$ & 8.98 & 1 & 1 & 1911 & 1911 & NGA_NGC4594 \\
\hline 300 & NGC4605 & 124000.3 & 613629 & 55.47 & 5.47 & $\operatorname{trgb}$ & 10.89 & 5 & 1 & 1654 & 1654 & GI1_047078_NGC4605 \\
\hline 301 & NGC4618 & 124132.7 & 410904 & 75.83 & 7.8 & $\mathrm{v}$ (flow) & 11.22 & 8 & 1 & 3242 & 3242 & NGA_NGC4625 \\
\hline 302 & NGC4625 & 124152.6 & 411626 & 75.72 & 8.7 & $\mathrm{v}$ (flow) & 12.92 & 9 & 1 & 3242 & 3242 & NGA_NGC4625 \\
\hline 303 & NGC4631 & 124208.0 & 323226 & 84.22 & 8.1 & $\mathrm{v}$ (flow) & 9.8 & 7 & 1 & 1465 & 1465 & NGA_NGC4631 \\
\hline 304 & UGC7866 & 124215.1 & 383012 & 78.46 & 4.57 & $\operatorname{trgb}$ & 13.7 & 10 & 1 & 1680 & 1680 & GI1_047079_IC3687 \\
\hline 305 & NGC4656 & 124357.7 & 321005 & 84.70 & 8.6 & $\mathrm{v}$ (flow) & 10.96 & 9 & 1 & 1672 & 3376 & GI1_047080_NGC4656 \\
\hline 306 & UGC7916 & 124425.1 & 342312 & 82.59 & 8.2 & $\mathrm{v}$ (flow) & 15 & 10 & 1 & 1349 & 1349 & GI1_047081_UGC07916 \\
\hline 307 & ESO381-G020 & 124600.4 & -335017 & 29.02 & 5.44 & $\operatorname{trgb}$ & 14.7 & 10 & 0 & 94 & 94 & AISCHV4_432_45330 \\
\hline 308 & UGCA298 & 124655.4 & 263351 & 88.85 & 10.3 & $\mathrm{v}$ (flow) & 15.6 & -3 & 0 & 116 & 116 & AISCHV4_113_12985 \\
\hline 309 & UGC7950 & 124656.4 & 513646 & 65.50 & 7.9 & $\mathrm{v}$ (flow) & 15.1 & 10 & 1 & 1664 & 1664 & GI1_047082_UGC07950 \\
\hline 310 & UGC7949 & 124659.8 & 362835 & 80.60 & 9.9 & bs & 15.12 & 10 & 1 & 1950 & 2846 & GI1_047083_UGC07949 \\
\hline 311 & NGC4707 & 124822.9 & 510953 & 65.96 & 7.4 & $\mathrm{v}$ (flow) & 13.4 & 9 & 1 & 1664 & 1664 & GI1_047082_UGC07950 \\
\hline 312 & NGC4736 & 125053.0 & 410714 & 76.01 & 4.66 & $\operatorname{trgb}$ & 9.0 & 2 & 1 & 3994 & 3994 & NGA_NGC4736 \\
\hline 313 & UGC8024 & 125405.2 & 270855 & 89.41 & 4.3 & bs & 13.9 & 10 & 1 & 1442 & 1442 & NGA_DDO154 \\
\hline 314 & UGC8055 & 125604.3 & 034843 & 66.66 & 6.6 & $\mathrm{v}$ (flow) & 17.00 & 10 & 0 & 110 & 110 & AISCHV4_228_13578 \\
\hline 315 & NGC4826 & 125643.7 & 214052 & 84.42 & 7.5 & $\mathrm{sbf}$ & 9.36 & 2 & 1 & 1330 & 5813 & WDST_GD_153 \\
\hline 316 & UGC8091 & 125840.4 & 141303 & 76.98 & 2.08 & $\operatorname{trgb}$ & 14.68 & 10 & 1 & 110 & 110 & AISCHV4_223_13511 \\
\hline 317 & UGC8146 & 130208.1 & 584205 & 58.37 & 10.6 & $\mathrm{v}$ (flow) & 14.4 & 6 & 0 & 6599 & 6599 & GI2_025004_PG1259p593 \\
\hline 318 & UGCA319 & 130214.4 & -171415 & 45.56 & 7.4 & $\mathrm{v}$ (flow) & 15.0 & 9 & 1 & 1456 & 7086 & GI1_047084_UGCA319 \\
\hline
\end{tabular}


Table 1

(Continued)

\begin{tabular}{|c|c|c|c|c|c|c|c|c|c|c|c|c|}
\hline$(1)$ & $\begin{array}{c}\text { Galaxy Name } \\
\text { (2) }\end{array}$ & $\begin{array}{c}\text { R.A. } \\
(\mathrm{J} 2000) \\
(3)\end{array}$ & $\begin{array}{l}\text { Decl. } \\
(\mathrm{J} 2000) \\
(4)\end{array}$ & $\begin{array}{c}b \\
(\mathrm{deg}) \\
(5) \\
\end{array}$ & $\begin{array}{c}\mathrm{D} \\
(\mathrm{Mpc}) \\
(6)\end{array}$ & $\begin{array}{l}\text { Method } \\
\text { (7) }\end{array}$ & $\begin{array}{c}B \\
(\mathrm{mag}) \\
(8)\end{array}$ & (9) & $\begin{array}{l}\text { Local Volume Legacy } \\
\qquad(10)\end{array}$ & $\begin{array}{l}t_{\text {fuv }} \\
(\mathrm{s}) \\
(11)\end{array}$ & $\begin{array}{l}t_{\text {nuv }} \\
(\mathrm{s}) \\
(12) \\
\end{array}$ & $\begin{array}{l}\text { Tile Name } \\
\text { (13) }\end{array}$ \\
\hline 319 & UGCA320 & 130316.8 & -172523 & 45.36 & 7.2 & $\mathrm{v}$ (flow) & 13.5 & 9 & 1 & 1456 & 7086 & GI1_047084_UGCA319 \\
\hline 320 & NGC4945 & 130527.5 & -492806 & 13.34 & 3.6 & mem & 9.3 & 6 & 0 & $\ldots$ & $\ldots$ & \\
\hline 321 & UGC8188 & 130549.5 & 373618 & 79.09 & 4.49 & ceph & 12 & 9 & 1 & 1666 & 1666 & GI1_047085_IC4182 \\
\hline 322 & UGC8201 & 130624.8 & 674225 & 49.36 & 4.57 & $\operatorname{trgb}$ & 12.8 & 10 & 1 & 1701 & 1701 & NGA_DDO165 \\
\hline 323 & MCG-03-34-002 & 130756.6 & -164121 & 46.00 & 10.2 & $\mathrm{v}$ (flow) & 14.8 & 4 & 1 & 1658 & 1658 & GI4_095039_MCG_03_34_002 \\
\hline 324 & UGC8215 & 130803.6 & 464941 & 70.03 & 4.55 & $\operatorname{trgb}$ & 16.08 & 10 & 0 & 1584 & 3105 & GI1_047086_UGC08215 \\
\hline 325 & UGC8245 & 130834.2 & 785613 & 38.16 & 3.6 & $\mathrm{v}$ (flow) & 15.2 & 10 & 1 & 1549 & 1549 & GI4_095026_UGC08245 \\
\hline 326 & NGC5023 & 131212.1 & 440220 & 72.58 & 5.4 & bs & 12.8 & 6 & 1 & 1586 & 4723 & GI1_047087_NGC5023 \\
\hline 327 & CGCG217-018 & 131251.8 & 403235 & 75.87 & 8.2 & $\mathrm{v}$ (flow) & 15.1 & 10 & 1 & 1321 & 2901 & GI4_095027_CGCG217_018 \\
\hline 328 & UGC8308 & 131322.7 & 461913 & 70.32 & 4.19 & $\operatorname{trgb}$ & 15.54 & 10 & 0 & 1576 & 3105 & GI1_047088_UGC08308 \\
\hline 329 & UGC8313 & 131354.1 & 421236 & 74.24 & 8.7 & $\mathrm{v}$ (flow) & 14.78 & 5 & 1 & 1655 & 1655 & NGA_NGC5055 \\
\hline 330 & UGC8320 & 131427.9 & 455509 & 70.66 & 4.33 & $\operatorname{trgb}$ & 13.11 & 10 & 1 & 1576 & 3105 & GI1_047088_UGC08308 \\
\hline 331 & UGC8331 & 131530.3 & 472956 & 69.09 & 8.2 & bs & 14.31 & 10 & 1 & 1628 & 2503 & GI1_047090_UGC08331 \\
\hline 332 & NGC5055 & 131549.2 & 420149 & 74.29 & 7.5 & $\mathrm{v}$ (flow) & 9.31 & 4 & 1 & 1655 & 1655 & NGA_NGC5055 \\
\hline 333 & NGC5068 & 131854.6 & -210220 & 41.38 & 6.2 & $\mathrm{v}$ (flow) & 10.7 & 6 & 1 & 1601 & 4783 & GI1_047091_NGC5068 \\
\hline 334 & NGC5102 & 132157.6 & -363749 & 25.84 & 3.40 & $\operatorname{trgb}$ & 10.35 & -3 & 0 & & & \\
\hline 335 & NGC5128 & 132527.6 & -430109 & 19.42 & 3.66 & $\operatorname{trgb}$ & 7.84 & -2 & 0 & 20073 & 30429 & NGA_Cen_A_Jet \\
\hline 336 & IC4247 & 132644.4 & -302145 & 31.89 & 4.97 & $\operatorname{trgb}$ & 14.6 & 2 & 1 & 1663 & 1663 & GI4_095028_IC4247 \\
\hline 337 & ESO324-G024 & 132737.3 & -412850 & 20.88 & 3.73 & $\operatorname{trgb}$ & 12.9 & 10 & 0 & 121 & 121 & AISCHV4_471__45528 \\
\hline 338 & NGC5204 & 132936.2 & 582506 & 58.00 & 4.65 & $\operatorname{trgb}$ & 11.7 & 9 & 1 & 1488 & 1488 & GI4_095029_NGC5204 \\
\hline 339 & NGC5194 & 132952.7 & 471143 & 68.56 & 8.0 & mem & 8.96 & 4 & 1 & 2520 & 2520 & NGA_M51 \\
\hline 340 & NGC5195 & 132958.7 & 471605 & 68.49 & 8.0 & sbf & 10.45 & 2 & 1 & 2520 & 2520 & NGA_M51 \\
\hline 341 & UGC8508 & 133044.4 & 545436 & 61.31 & 2.58 & $\operatorname{trgb}$ & 13.94 & 10 & 1 & $\ldots$ & $\ldots$ & $\ldots$ \\
\hline 342 & SBS1331+493 & 133322.9 & 490606 & 66.58 & 9.3 & $\mathrm{v}$ (flow) & 16.5 & 11 & 0 & $\ldots$ & $\ldots$ & \\
\hline 343 & NGC5206 & 133343.8 & -480904 & 14.12 & 3.6 & mem & 12 & -3 & 0 & $\ldots$ & $\ldots$ & \\
\hline 344 & NGC5229 & 133402.7 & 475455 & 67.61 & 5.1 & bs & 14.18 & 7 & 1 & 2757 & 2757 & GI1_047092_NGC5229 \\
\hline 345 & NGC5238 & 133442.7 & 513651 & 64.19 & 5.2 & bs & 13.60 & 8 & 1 & 1618 & 1618 & GI1_047093_NGC5238 \\
\hline 346 & ESO270-G017 & 133447.3 & -453251 & 16.66 & 8.9 & $\mathrm{tf}$ & 12 & 4 & 0 & $\ldots$ & $\ldots$ & \\
\hline 347 & [KK98]208 & 133635.5 & -293417 & 32.28 & 4.68 & $\operatorname{trgb}$ & 14.30 & -1 & 1 & 13361 & 13366 & GI3_050007_NGC5236 \\
\hline 348 & NGC5236 & 133700.8 & -295159 & 31.97 & 4.47 & ceph & 8.20 & 5 & 1 & 13361 & 13366 & GI3_050007_NGC5236 \\
\hline 349 & ESO444-G084 & 133720.1 & -280246 & 33.74 & 4.61 & $\operatorname{trgb}$ & 15.5 & 10 & 1 & 1571 & 6357 & GI1_047094_ESOT444_G084 \\
\hline 350 & UGC8638 & 133919.4 & 244632 & 78.98 & 4.27 & $\operatorname{trgb}$ & 15 & 10 & 1 & 1604 & 1604 & GI1_047095_UGC08638 \\
\hline 351 & UGC8651 & 133953.8 & 404421 & 73.12 & 3.14 & $\operatorname{trgb}$ & 14.45 & 10 & 1 & 1690 & 1690 & GI1_047096_UGC08651 \\
\hline 352 & NGC5253 & 133955.9 & -313824 & 30.10 & 3.15 & ceph & 10.9 & 11 & 1 & 1657 & 1657 & GI4_095049_NGC5253 \\
\hline 353 & IC4316 & 134018.4 & -285332 & 32.77 & 4.40 & $\operatorname{trgb}$ & 14.6 & 10 & 0 & 217 & 217 & AISCHV4_491_35380 \\
\hline 354 & NGC5264 & 134136.9 & -295450 & 31.71 & 4.53 & $\operatorname{trgb}$ & 12.6 & 9 & 1 & 1878 & 1878 & GI4_095030_NGC5264 \\
\hline 355 & UGC8683 & 134232.4 & 393930 & 73.58 & 9.6 & $\mathrm{v}$ (flow) & 15.6 & 10 & 0 & 2400 & 5044 & GI1_047097_UGC08683 \\
\hline 356 & ESO325-G011 & 134500.5 & -415140 & 19.91 & 3.40 & $\operatorname{trgb}$ & 14.0 & 10 & 0 & $\ldots$ & $\ldots$ & $\ldots$ \\
\hline 357 & ESO383-G087 & 134917.8 & -360342 & 25.36 & 3.45 & $\operatorname{trgb}$ & 11.7 & 8 & 0 & $\ldots$ & $\ldots$ & $\ldots$ \\
\hline 358 & ESO383-G091 & 135032.3 & -371720 & 24.10 & 3.6 & mem & 14.43 & 7 & 0 & 136 & 136 & AISCHV4_466_45755 \\
\hline 359 & UGC8760 & 135050.6 & 380109 & 73.45 & 3.22 & $\operatorname{trgb}$ & 14.45 & 10 & 1 & 2425 & 2425 & GI1_047098_UGC08760 \\
\hline 360 & UGC8837 & 135445.7 & 535403 & 60.80 & 8.3 & bs & 13.71 & 10 & 1 & $\ldots$ & $\ldots$ & \\
\hline 361 & UGC8833 & 135448.7 & 355015 & 73.96 & 3.08 & $\operatorname{trgb}$ & 15.15 & 10 & 1 & 1234 & 2706 & GI1_047099_UGC08833 \\
\hline 362 & ESO384-G016 & 135701.5 & -352002 & 25.65 & 4.53 & $\operatorname{trgb}$ & 15.0 & 10 & 0 & 111 & 111 & AISCHV4_351_45685 \\
\hline 363 & NGC5457 & 140312.5 & 542055 & 59.77 & 6.70 & ceph & 8.31 & 6 & 1 & 1040 & 1040 & NGA_M101 \\
\hline 364 & NGC5408 & 140321.0 & -412244 & 19.50 & 4.81 & $\operatorname{trgb}$ & 12.2 & 10 & 0 & $\ldots$ & $\ldots$ & \\
\hline 365 & NGC5474 & 140501.5 & 533945 & 60.19 & 7.2 & bs & 11.82 & 6 & 1 & 1604 & 1604 & NGA_NGC5474 \\
\hline 366 & NGC5477 & 140533.1 & 542739 & 59.49 & 7.7 & bs & 14.24 & 9 & 1 & 1040 & 1040 & NGA_M101 \\
\hline 367 & KKR03 & 140710.7 & 350337 & 71.99 & 1.97 & $\operatorname{trgb}$ & 17.84 & 10 & 1 & 2090 & 2090 & GI3_061004_KKR03 \\
\hline 368 & Circinus & 141309.3 & -652021 & -3.81 & 2.8 & tf & 12.1 & 3 & 0 & $\ldots$ & $\ldots$ & $\ldots$ \\
\hline 369 & KUG1413+573 & 141509.4 & 570515 & 56.56 & 7.4 & mem & 16.10 & 10 & 0 & 108 & 108 & AISCHV4_101_11865 \\
\hline 370 & UGC9128 & 141556.5 & 230319 & 70.46 & 2.21 & $\operatorname{trgb}$ & 14.46 & 10 & 1 & 1986 & 5293 & GI4_016007_DDO187 \\
\hline 371 & SBS1415+437 & 141701.4 & 433005 & 66.20 & 9.5 & $\mathrm{v}$ (flow) & 18 & 11 & 0 & 112 & 112 & AISCHV3_109_01380 \\
\hline 372 & NGC5585 & 141948.2 & 564346 & 56.48 & 5.7 & bs & 11.2 & 7 & 1 & 2936 & 2936 & GI4_095032_NGC5585 \\
\hline 373 & UGC9211 & 142232.2 & 452302 & 64.28 & 10.7 & $\mathrm{v}$ (flow) & 14.80 & 10 & 0 & 96 & 96 & AISCHV4_105_10645 \\
\hline 374 & NGC5608 & 142317.9 & 414633 & 66.19 & 10.2 & $\mathrm{v}$ (flow) & 14.1 & 10 & 0 & 121 & 121 & AISCHV3_109_01380 \\
\hline 375 & UGC9240 & 142443.4 & 443133 & 64.48 & 2.79 & $\operatorname{trgb}$ & 13.31 & 10 & 1 & 2737 & 4209 & GI1_047101_UGC09240 \\
\hline 376 & UKS1424-460 & 142803.7 & -461806 & 13.38 & 3.58 & $\operatorname{trgb}$ & 16.50 & 10 & 0 & $\ldots$ & $\ldots$ & $\ldots$ \\
\hline 377 & ESO222-G010 & 143502.6 & -492514 & 10.05 & 5.4 & $\mathrm{v}$ (flow) & 16.33 & 10 & 0 & $\ldots$ & $\ldots$ & $\ldots$ \\
\hline 378 & UGC9405 & 143524.4 & 571519 & 54.71 & 8.0 & bs & 14.57 & 10 & 1 & 1523 & 1523 & GI4_095033_UGC09405 \\
\hline 379 & MRK475 & 143905.4 & 364821 & 65.31 & 9.0 & $\mathrm{v}$ (flow) & 15.5 & 11 & 1 & 1932 & 3618 & GI1_047102_MRK0475 \\
\hline 380 & ESO272-G025 & 144325.5 & -444219 & 13.75 & 5.6 & $\mathrm{v}$ (flow) & 14.93 & 8 & 0 & $\ldots$ & $\ldots$ & $\ldots$ \\
\hline 381 & UGC9497 & 144412.8 & 423744 & 62.37 & 10.0 & $\mathrm{v}$ (flow) & 15.9 & 6 & 0 & 192 & 192 & AISCHV4_110_10896 \\
\hline 382 & NGC5832 & 145745.7 & 714056 & 42.16 & 8.8 & $\mathrm{v}$ (flow) & 12.9 & 3 & 1 & 1432 & 1432 & GI1_047103_NGC5832 \\
\hline
\end{tabular}


Table 1

(Continued)

\begin{tabular}{|c|c|c|c|c|c|c|c|c|c|c|c|c|}
\hline No. & Galaxy Name & $\begin{array}{c}\text { R.A. } \\
\text { (J2000) } \\
(3)\end{array}$ & $\begin{array}{c}\text { Decl. } \\
(\mathrm{J} 2000) \\
(4)\end{array}$ & $\begin{array}{c}b \\
(\mathrm{deg}) \\
(5)\end{array}$ & $\begin{array}{c}\mathrm{D} \\
(\mathrm{Mpc}) \\
(6)\end{array}$ & Method & $\begin{array}{c}B \\
(\mathrm{mag}) \\
(8)\end{array}$ & (9) & Local Volume Legacy & $\begin{array}{c}t_{\text {fuv }} \\
(\mathrm{s}) \\
(11)\end{array}$ & $\begin{array}{c}t_{\text {nuv }} \\
(\mathrm{s}) \\
(12)\end{array}$ & Tile Name \\
\hline 383 & ESO223-G009 & 150108.7 & -481726 & 9.17 & 6.49 & $\operatorname{trgb}$ & 13 & 10 & 0 & $\ldots$ & $\ldots$ & $\ldots$ \\
\hline 384 & UGC9660 & 150109.3 & 444153 & 58.73 & 10.2 & $\mathrm{v}$ (flow) & 14.2 & 4 & 0 & 198 & 198 & AISCHV4_105_10892 \\
\hline 385 & ESO274-G001 & 151413.6 & -464836 & 9.34 & 3.09 & $\operatorname{trgb}$ & 11.7 & 7 & 0 & $\ldots$ & $\ldots$ & $\ldots$ \\
\hline 386 & NGC5949 & 152800.7 & 644547 & 44.97 & 8.5 & $\mathrm{v}$ (flow) & 13.4 & 4 & 1 & 1578 & 3822 & GI1_047104_NGC5949 \\
\hline 387 & UGC9893 & 153257.3 & 462710 & 52.90 & 10.9 & $\mathrm{v}$ (flow) & 15 & 7 & 0 & 8379 & 8379 & GI4_015005_IZW115 \\
\hline 388 & UGC9992 & 154147.8 & 671515 & 42.37 & 8.6 & $\mathrm{v}$ (flow) & 14.86 & 10 & 1 & 2239 & 3447 & GI1_047105_UGC09992 \\
\hline 389 & LEDA100404 & 160858.9 & 173025 & 43.60 & 6.8 & $\mathrm{v}$ (flow) & 17.4 & 9 & 0 & $\ldots$ & $\ldots$ & $\ldots$ \\
\hline 390 & ESO137-G018 & 162059.2 & -602916 & -7.43 & 6.40 & $\operatorname{trgb}$ & 12.2 & 5 & 0 & $\ldots$ & $\ldots$ & $\ldots$ \\
\hline 391 & ESO179-IG013 & 164720.0 & -572628 & -7.90 & 9.0 & $\mathrm{v}$ (flow) & 15 & 9 & 0 & $\ldots$ & $\ldots$ & $\ldots$ \\
\hline 392 & UGC10669 & 170025.3 & 701724 & 34.68 & 9.2 & $\mathrm{v}$ (flow) & 16.3 & 10 & 0 & 181 & 181 & AISCHV4_002_09801 \\
\hline 393 & UGC10736 & 170805.0 & 692750 & 34.27 & 9.8 & $\mathrm{v}$ (flow) & 15.1 & 8 & 0 & 1561 & 1561 & GI1_047106_UGC10736 \\
\hline 394 & IC4662 & 174706.4 & -643825 & -17.85 & 2.44 & $\operatorname{trgb}$ & 11.7 & 10 & 0 & 158 & 158 & AISCHV4_476_43915 \\
\hline 395 & NGC6503 & 174927.1 & 700840 & 30.64 & 5.27 & $\operatorname{trgb}$ & 10.91 & 6 & 1 & 1597 & 4303 & GI1_097010_NGC6503 \\
\hline 396 & ESO140-G019 & 182246.4 & -621613 & -20.61 & 10.8 & v(flow) & 16.8 & 10 & 0 & $\ldots$ & $\ldots$ & $\ldots$ \\
\hline 397 & IC4710 & 182838.1 & -665854 & -22.65 & 7.7 & $\mathrm{v}$ (flow) & 12.5 & 9 & 0 & $\ldots$ & $\ldots$ & $\ldots$ \\
\hline 398 & NGC6689 & 183450.1 & 703127 & 26.83 & 9.8 & $\mathrm{v}$ (flow) & 13.1 & 6 & 0 & $\ldots$ & $\ldots$ & $\ldots$ \\
\hline 399 & ESO104-G022 & 185541.2 & -644839 & -24.81 & 8.7 & $\mathrm{v}$ (flow) & 15.5 & 10 & 0 & $\ldots$ & $\ldots$ & $\ldots$ \\
\hline 400 & NGC6744 & 190946.2 & -635125 & -26.14 & 9.4 & $\mathrm{v}$ (flow) & 9.1 & 4 & 0 & 1290 & 1290 & LGAL_NGC6744n \\
\hline 401 & ESO104-G044 & 191123.1 & -641309 & -26.38 & 8.4 & $\mathrm{v}$ (flow) & 14.9 & 9 & 0 & 1290 & 1290 & LGAL_NGC6744n \\
\hline 402 & NGC6789 & 191641.9 & 635818 & 21.52 & 3.60 & $\operatorname{trgb}$ & 14 & 10 & 0 & 1403 & 1403 & NGA_NGC6789 \\
\hline 403 & ESO594-G004 & 192959.0 & -174041 & -16.29 & 1.04 & $\operatorname{trgb}$ & 13.99 & 10 & 0 & 2898 & 2898 & GI4_016010_SAGDIG \\
\hline 404 & IC4870 & 193737.6 & -654843 & -29.27 & 9.9 & $\mathrm{v}$ (flow) & 14.4 & 10 & 0 & 216 & 216 & AISCHV4_373_44415 \\
\hline 405 & NGC6822 & 194456.6 & -144721 & -18.40 & 0.50 & ceph & 9.31 & 10 & 0 & 4590 & 6132 & NGA_NGC6822 \\
\hline 406 & IC4951 & 200931.2 & -615047 & -32.84 & 9.3 & $\mathrm{v}$ (flow) & 13.9 & 8 & 1 & 2401 & 2401 & GI4_095050_IC4951 \\
\hline 407 & UGC11583 & 203015.3 & 602625 & 12.31 & 5.9 & bs & 15.70 & 10 & 0 & $\ldots$ & $\ldots$ & $\ldots$ \\
\hline 408 & LEDA166192 & 203032.9 & 602117 & 12.23 & 5.9 & bs & 16.50 & 8 & 0 & $\ldots$ & $\ldots$ & $\ldots$ \\
\hline 409 & LEDA166193 & 203132.0 & 604844 & 12.39 & 5.9 & bs & 16.70 & 10 & 0 & $\ldots$ & $\ldots$ & $\ldots$ \\
\hline 410 & NGC6946 & 203452.3 & 600914 & 11.67 & 5.9 & bs & 9.61 & 6 & 0 & 885 & 885 & UVX_NGC6946 \\
\hline 411 & KKR55 & 204520.8 & 602440 & 10.78 & 5.9 & bs & 18 & 10 & 0 & $\ldots$ & $\ldots$ & $\ldots$ \\
\hline 412 & DDO210 & 204651.8 & -125053 & -31.34 & 0.94 & $\operatorname{trgb}$ & 14.1 & 10 & 1 & 1695 & 1695 & GI1_047107_DDO210 \\
\hline 413 & KKR56 & 204824.1 & 583706 & 9.37 & 5.9 & bs & 17.60 & 10 & 0 & $\ldots$ & $\ldots$ & $\ldots$ \\
\hline 414 & CEPHEUS1 & 205110.6 & 565325 & 8.01 & 5.9 & mem & 15.40 & 10 & 0 & $\ldots$ & $\ldots$ & $\ldots$ \\
\hline 415 & IC5052 & 205206.3 & -691213 & -35.81 & 5.9 & $\mathrm{v}($ flow $)$ & 11.8 & 7 & 1 & 2666 & 2666 & GI4_095045_IC5052 \\
\hline 416 & KKR59 & 210324.2 & 571714 & 6.99 & 5.9 & bs & 15.70 & 10 & 0 & $\ldots$ & $\ldots$ & $\cdots$ \\
\hline 417 & KKR60 & 210551.3 & 571232 & 6.69 & 5.9 & mem & 18.00 & 10 & 0 & $\ldots$ & $\ldots$ & $\ldots$ \\
\hline 418 & NGC7064 & 212903.0 & -524603 & -44.82 & 9.9 & $\mathrm{v}$ (flow) & 13.1 & 5 & 1 & 1971 & 1971 & GI1_047108_NGC7064 \\
\hline 419 & NGC7090 & 213628.6 & -543324 & -45.38 & 10.4 & $\mathrm{v}$ (flow) & 11.3 & 5 & 1 & 3010 & 3091 & GI1_047109_NGC7090 \\
\hline 420 & IC5152 & 220241.9 & -511744 & -50.19 & 1.97 & $\operatorname{trgb}$ & 10.7 & 10 & 1 & 4076 & 4077 & GI4_095034_IC5152 \\
\hline 421 & UGC11891 & 220333.9 & 434457 & -9.36 & 10.8 & $\mathrm{v}$ (flow) & 15.3 & 10 & 0 & $\ldots$ & $\ldots$ & $\ldots$ \\
\hline 422 & ESO238-G005 & 222230.0 & -482418 & -54.24 & 8.9 & $\mathrm{v}$ (flow) & 15.60 & 10 & 0 & 2418 & 2418 & GI1_047110_ESO238_G005 \\
\hline 423 & IC5256 & 224945.8 & -684126 & -44.73 & 10.8 & $\mathrm{v}$ (flow) & 14.6 & 8 & 1 & 1856 & 1856 & GI4_095040_IC5256 \\
\hline 424 & NGC7640 & 232206.6 & 405043 & -18.94 & 8.9 & $\mathrm{v}$ (flow) & 11.9 & 5 & 0 & $\ldots$ & $\ldots$ & $\ldots$ \\
\hline 425 & UGC12588 & 232442.4 & 412048 & -18.64 & 9.5 & $\mathrm{v}$ (flow) & 14.4 & 8 & 0 & $\ldots$ & $\ldots$ & $\ldots$ \\
\hline 426 & UGCA438 & 232627.5 & -322320 & -70.86 & 2.18 & $\operatorname{trgb}$ & 14.7 & 10 & 1 & 1693 & 1693 & NGA_UGCA438 \\
\hline 427 & ESO347-G017 & 232656.0 & -372049 & -69.49 & 9.4 & $\mathrm{v}$ (flow) & 14.41 & 9 & 1 & 2252 & 2252 & GI4_095035_ESO347_G017 \\
\hline 428 & UGC12613 & 232836.2 & 144435 & -43.55 & 0.76 & $\operatorname{trgb}$ & 12.5 & 10 & 1 & 1576 & 2932 & MISDR2_28664_0746 \\
\hline 429 & UGC12632 & 232958.7 & 405925 & -19.31 & 9.6 & $\mathrm{v}$ (flow) & 12.8 & 9 & 0 & 196 & 196 & AISCHV3_037_08795 \\
\hline 430 & IC5332 & 233427.4 & -360605 & -71.37 & 9.5 & $\mathrm{v}$ (flow) & 11.2 & 7 & 1 & 2646 & 2646 & GI4_095036_IC5332 \\
\hline 431 & NGC7713 & 233615.4 & -375619 & -70.88 & 9.3 & v(flow) & 11.5 & 7 & 1 & $\ldots$ & $\ldots$ & $\ldots$ \\
\hline 432 & UGC12713 & 233814.4 & 304229 & -29.58 & 7.7 & $\mathrm{v}$ (flow) & 14.91 & 0 & 0 & 2896 & 2896 & GI3_089016_MRK328 \\
\hline 433 & UGCA442 & 234345.5 & -315722 & -74.53 & 4.27 & $\operatorname{trgb}$ & 13.6 & 9 & 1 & 871 & 871 & GI4_095037_UGCA442 \\
\hline 434 & ESO348-G009 & 234923.5 & -374619 & -73.17 & 8.6 & $\mathrm{v}$ (flow) & 17 & 10 & 0 & 2534 & 4032 & GI1_047111_ESO348_G009 \\
\hline 435 & ESO149-G003 & 235202.8 & -523440 & -62.24 & 6.4 & $\mathrm{tf}$ & 15.0 & 10 & 1 & 1563 & 1622 & GI1_047112_ESO149_G003 \\
\hline 436 & NGC7793 & 235749.7 & -323530 & -77.17 & 3.91 & $\operatorname{trgb}$ & 9.63 & 7 & 1 & 1552 & 1552 & NGA_NGC7793 \\
\hline 1 & ESO410-G005 & 001531.4 & 321047 & -80.71 & 2.01 & $\operatorname{trgb}$ & 14.95 & -1 & 1 & 1501 & 1501 & GI3_061005_ESO410_G005 \\
\hline 2 & SCULPTOR-DE1 & 002351.7 & -244218 & -83.34 & 4.19 & $\operatorname{trgb}$ & 17.8 & -3 & 1 & 1584 & 1584 & GI3_061022_SculptordE1 \\
\hline 3 & ESO294-G010 & 002633.4 & -415119 & -74.42 & 1.94 & $\operatorname{trgb}$ & 15.52 & -3 & 1 & 2703 & 2703 & GI3_061006_ESO294_G010 \\
\hline 4 & ESO540-G030 & 004920.9 & -180432 & -80.93 & 3.33 & $\operatorname{trgb}$ & 16.46 & -1 & 1 & 1656 & 1656 & GI3_061012_ESO540_G030 \\
\hline 5 & ESO540-G032 & 005024.3 & -195424 & -82.77 & 3.54 & $\operatorname{trgb}$ & 16.6 & -3 & 1 & 1247 & 1247 & GI3_061014_ESO540_G032 \\
\hline 6 & UGC521 & 005112.2 & 120131 & -50.85 & 11.3 & $\mathrm{v}$ (flow) & 15.31 & 10 & 1 & 1613 & 1613 & GI1_078003_UGC00521 \\
\hline 7 & NGC0404 & 010927.0 & 354304 & -27.01 & 3.05 & $\operatorname{trgb}$ & 11.51 & -1 & 1 & 2472 & 2472 & NGA_NGC0404_CEN \\
\hline 8 & IC2049 & 041204.3 & -583325 & -43.35 & 16.7 & $\mathrm{v}($ flow $)$ & 15.19 & 7 & 1 & 2602 & 2603 & GI1_047025_IC2049 \\
\hline 9 & UGCA133 & 073411.4 & 665310 & 28.96 & 3.10 & $\operatorname{trgb}$ & 15.80 & -3 & 1 & 1696 & 1696 & GI3_061009_UGCA133 \\
\hline
\end{tabular}


Table 1

(Continued)

\begin{tabular}{|c|c|c|c|c|c|c|c|c|c|c|c|c|}
\hline (1) & $\begin{array}{c}\text { Galaxy Name } \\
(2) \\
\end{array}$ & $\begin{array}{c}\text { R.A. } \\
(\mathrm{J} 2000) \\
(3)\end{array}$ & $\begin{array}{c}\text { Decl. } \\
(\mathrm{J} 2000) \\
(4)\end{array}$ & $\begin{array}{c}b \\
(\mathrm{deg}) \\
(5)\end{array}$ & $\begin{array}{c}\mathrm{D} \\
(\mathrm{Mpc}) \\
(6)\end{array}$ & $\begin{array}{c}\text { Method } \\
\text { (7) } \\
\end{array}$ & $\begin{array}{c}B \\
(\mathrm{mag}) \\
(8)\end{array}$ & (9) & $\begin{array}{l}\text { Local Volume Legacy } \\
\qquad(10)\end{array}$ & $\begin{array}{l}t_{\text {fuv }} \\
(\mathrm{s}) \\
(11) \\
\end{array}$ & $\begin{array}{l}t_{\text {nuv }} \\
(\mathrm{s}) \\
(12) \\
\end{array}$ & $\begin{array}{l}\text { Tile Name } \\
\text { (13) }\end{array}$ \\
\hline 10 & F8D1 & 094447.1 & 672619 & 40.95 & 3.66 & $\operatorname{trgb}$ & 16.14 & -3 & 1 & 1693 & 1693 & GI3_061016_KK77 \\
\hline 12 & LEDA166101 & 095010.5 & 673024 & 41.36 & 3.55 & $\operatorname{trgb}$ & 16.94 & -3 & 1 & 1693 & 1693 & GI3_061016_KK77 \\
\hline 13 & ARPSLOOP & 095732.6 & 691660 & 40.92 & 3.78 & $\operatorname{trgb}$ & 16.8 & 10 & 1 & 3075 & 3075 & NGA_M81andM82 \\
\hline 14 & ККH057 & 100015.9 & 631106 & 44.55 & 3.90 & $\operatorname{trgb}$ & 17.95 & -3 & 1 & 1694 & 1694 & MISDR1_00432_0487 \\
\hline 17 & UGC05442 & 100701.9 & 674939 & 42.48 & 3.72 & $\operatorname{trgb}$ & 15.78 & -3 & 1 & 3391 & 3391 & GI3_061018_UGC05442 \\
\hline 18 & IKN & 100805.9 & 682357 & 42.21 & 3.61 & $\operatorname{trgb}$ & 17.31 & -3 & 1 & 1699 & 1699 & NGA_NGC3077 \\
\hline 19 & HS98_117 & 102125.2 & 710651 & 41.30 & 3.82 & $\operatorname{trgb}$ & 17.01 & 10 & 1 & 101 & 101 & AISCHV4_118_00227 \\
\hline 20 & DDO078 & 102627.4 & 673916 & 43.00 & 3.66 & $\operatorname{trgb}$ & 15.8 & -3 & 1 & 138 & 138 & AISCHV4_118_00227 \\
\hline 21 & $\mathrm{BK} 06 \mathrm{~N}$ & 103429.8 & 660030 & 45.70 & 3.80 & $\operatorname{trgb}$ & 16.85 & -3 & 1 & 1508 & 1508 & GI3_061020_BK06N \\
\hline 22 & UGC6782 & 114857.2 & 235016 & 75.53 & 14.0 & bs & 15.07 & 9 & 1 & 2304 & 4756 & GI1_047052_ŪGC06782 \\
\hline
\end{tabular}

(This table is also available in a machine-readable form in the online journal.)

and $11 \mathrm{Mpc}$, where flow distance uncertainties $( \pm 15 \%)$ may scatter objects in and out of the volume. Such uncertainties, however, should not be significant for studies seeking to use the sample to statistically characterize the physical properties of local galaxies. As we previously commented in Paper I and in Dale et al. (2009), an inherent difficulty with efforts to construct a local volume-limited sample is that its membership will necessarily be fluid until accurate distance and photometric measurements are available for all of the galaxies that are within the volume and around its periphery.

Both the archival and newly obtained Spitzer imaging for the Local Volume Legacy sample have been uniformly processed (Dale et al. 2009). The full set of IRAC, MIPS, and available $\mathrm{H} \alpha$ and GALEX UV imaging for the Local Volume Legacy galaxies are all provided online (see footnote 13). UV photometry measured in apertures identical to those used for the Spitzer photometry and spectral energy distributions published in Dale et al. (2009) is provided in Table 3.

Column 13: GALEX "tile" name(s) giving information on the original program that requested observations for the galaxy.

\subsection{GALEX Photometry}

The procedures used to perform photometry closely follow those used by Gil de Paz \& Madore (2005), for ground-based optical imaging, and Gil de Paz et al. (2007) for the GALEX Atlas of Nearby Galaxies. Both total (asymptotic) magnitudes based on curve-of-growth analyses and aperture magnitudes are measured and reported in Tables 2 and 3. Details on the contents of the tables are given at the end of this section.

To prepare the images for photometry, foreground stars and background galaxies are masked in a two-step procedure. The IRAF task STARFIND is used to identify all point sources down to $4 \sigma$ significance (i.e., at the $G A L E X$ resolution, $\sim 5^{\prime \prime}$ ), and those with FUV-NUV $>1$ are flagged as foreground Galactic stars. These initial masks are then inspected by eye. The nuclei of a few early-type dwarf galaxies that were flagged by STARFIND are unmasked. Blue foreground stars are also identified during this inspection and added to the mask. We note that such stars are rare at the Galactic latitudes targeted by 11HUGS $\left(|b|>30^{\circ}\right)$ and are readily differentiated from stellar clusters or associations in the target galaxy. Background galaxies were also identified by visual inspection, based upon the morphology in available optical imaging and/or anomalous UV color. The masked pixels are replaced by values interpolated from the surrounding pixels.

To perform surface photometry and obtain total (asymptotic) magnitudes, knowledge of the sky background level is required. We measure the background using pixels in equal-area regions distributed in azimuth around the galaxy in two concentric annuli and compute the average sky background, local background standard deviation, and standard deviation of the mean across the field. The annuli are chosen to lie well beyond the RC3 (Third Reference Catalog of Bright Galaxies; de Vaucouleurs et al. 1991) $B$-band 25 mag $\operatorname{arcsec}^{-2}$ isophote (typically 2-4 times $D_{25}$ ), where no emission from the galaxy is detected. The image used in this calculation is completely masked of all objects, including the target galaxy itself. The masks for this sky calculation are created from objects detected in the GALEX pipeline and extracted from the GALEX merged catalog file, MCAT.

The surface photometry is carried out on the masked, locally interpolated image using the IRAF task ELLIPSE, with fixed central coordinates, ellipticity, and position angle. The central coordinates are given in Table 1 . The position angles, and the semimajor and semiminor axis sizes on which the ellipticities are based, are given in Table 2. Values for these parameters are drawn from NED and are generally based on $B$-band measurements published in the RC3. The resulting elliptical apertures were individually checked by eye to ensure that they adequately described the galaxy on optical DSS images and were correctly centered on the GALEX images. Manual adjustments to the values taken from NED were made as necessary.

Surface brightness profiles are computed by ELLIPSE, where errors in the azimuthally averaged surface brightnesses are taken into account, and include contributions from the photon noise and both high- and low-frequency errors in the background error budget (see Gil de Paz et al. 2007 and Gil de Paz \& Madore 2005 for more details). To determine the asymptotic magnitudes, the 
Table 2

GALEX Aperture and Asymptotic Magnitudes

\begin{tabular}{|c|c|c|c|c|c|c|c|c|c|c|c|c|c|c|c|}
\hline \multirow{2}{*}{$\begin{array}{l}\text { No. } \\
\text { (1) }\end{array}$} & \multirow{2}{*}{$\begin{array}{c}\text { Galaxy Name } \\
\text { (2) }\end{array}$} & \multirow{2}{*}{$\begin{array}{c}E(B-V) \\
\text { (3) }\end{array}$} & \multirow{2}{*}{$\begin{array}{l}a \\
(") \\
(4)\end{array}$} & \multirow{2}{*}{$\begin{array}{l}b \\
(") \\
(5)\end{array}$} & \multirow{2}{*}{$\begin{array}{l}\text { P.A. } \\
\text { (deg) } \\
(6)\end{array}$} & \multicolumn{2}{|c|}{$\begin{array}{l}\mathrm{FUV}_{\mathrm{ap}} \\
(\mathrm{mag})\end{array}$} & \multicolumn{2}{|c|}{$\begin{array}{l}\mathrm{NUV}_{\text {ap }} \\
(\mathrm{mag})\end{array}$} & \multicolumn{2}{|c|}{$\begin{array}{c}(\mathrm{FUV}- \\
\mathrm{NUV})_{a \mathrm{p}} \\
(\mathrm{mag})\end{array}$} & FUV & asym & NUV & \\
\hline & & & & & & (7) & (8) & (9) & $(10)$ & (11) & $(12)$ & (13) & (14) & (15) & (16) \\
\hline 1 & UGC12894 & 0.11 & 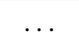 & & & & & & & & & & & & \\
\hline 2 & WLM & 0.04 & 516 & 180 & 4 & 12.48 & 0.05 & 12.35 & 0.03 & 0.13 & 0.06 & 12.49 & 0.05 & 12.38 & 0.03 \\
\hline 3 & ESO409-IG015 & 0.02 & 90 & 40 & -40 & 15.94 & 0.05 & 15.82 & 0.04 & 0.12 & 0.06 & 15.94 & 0.05 & 15.81 & 0.04 \\
\hline 4 & ESO349-G031 & 0.01 & 72 & 72 & 0 & 17.05 & 0.05 & 16.91 & 0.04 & 0.14 & 0.06 & 17.04 & 0.05 & 16.88 & 0.04 \\
\hline 5 & NGC24 & 0.02 & 258 & 58 & 46 & 14.04 & 0.05 & 13.77 & 0.03 & 0.27 & 0.06 & 14.01 & 0.05 & 13.75 & 0.03 \\
\hline 6 & NGC45 & 0.02 & 378 & 262 & -38 & 12.55 & 0.05 & 12.36 & 0.03 & 0.19 & 0.06 & 12.52 & 0.06 & 12.32 & 0.05 \\
\hline 7 & NGC55 & 0.01 & 1452 & 251 & -72 & 10.17 & 0.05 & 9.87 & 0.03 & 0.30 & 0.06 & 10.17 & 0.05 & 9.88 & 0.04 \\
\hline 8 & NGC59 & 0.02 & 150 & 75 & -53 & 16.06 & 0.06 & 15.31 & 0.03 & 0.75 & 0.07 & 16.08 & 0.06 & 15.31 & 0.03 \\
\hline 9 & MCG-04-02-003 & 0.02 & 36 & 36 & 0 & 17.46 & 0.05 & 17.18 & 0.03 & 0.28 & 0.06 & 17.19 & 0.41 & 17.00 & 0.17 \\
\hline 10 & IC10 & 1.56 & $\ldots$ & $\ldots$ & $\ldots$ & $\ldots$ & $\ldots$ & $\ldots$ & $\ldots$ & $\ldots$ & $\ldots$ & $\ldots$ & $\ldots$ & $\ldots$ & $\ldots$ \\
\hline 11 & ESO473-G024 & 0.02 & 108 & 65 & 29 & 16.75 & 0.06 & 16.71 & 0.07 & 0.04 & 0.09 & 16.77 & 0.06 & 16.75 & 0.07 \\
\hline 12 & AndIV & 0.09 & 54 & 42 & -40 & 17.65 & 0.06 & 17.15 & 0.04 & 0.50 & 0.07 & 17.37 & 0.14 & 16.48 & 0.18 \\
\hline 13 & NGC224 & 0.68 & $\ldots$ & $\ldots$ & $\ldots$ & $\ldots$ & $\ldots$ & $\ldots$ & $\ldots$ & $\ldots$ & $\ldots$ & $\ldots$ & $\ldots$ & $\ldots$ & $\ldots$ \\
\hline 14 & IC1574 & 0.02 & 120 & 46 & -5 & 16.56 & 0.05 & 16.07 & 0.03 & 0.49 & 0.06 & 16.55 & 0.05 & 16.05 & 0.03 \\
\hline 15 & NGC247 & 0.02 & 954 & 307 & -6 & 11.37 & 0.05 & 11.15 & 0.03 & 0.22 & 0.06 & 11.38 & 0.05 & 11.16 & 0.03 \\
\hline 16 & NGC253 & 0.02 & 1236 & 305 & 52 & 11.16 & 0.05 & 10.63 & 0.03 & 0.53 & 0.06 & 11.16 & 0.05 & 10.64 & 0.03 \\
\hline 17 & UGCA15 & 0.02 & 72 & 30 & 42 & 16.81 & 0.05 & 16.64 & 0.03 & 0.17 & 0.06 & 16.77 & 0.06 & 16.58 & 0.04 \\
\hline 18 & SMC & 0.04 & .. & $\ldots$ & $\ldots$ & $\ldots$ & & & & $\ldots$ & & & & $\ldots$ & \\
\hline 19 & NGC300 & 0.01 & 984 & 697 & -69 & 10.21 & 0.05 & 10.08 & 0.04 & 0.13 & 0.06 & 10.22 & 0.06 & 10.10 & 0.05 \\
\hline 20 & LGS3 & 0.04 & 48 & 48 & 0 & 19.16 & 0.11 & 18.36 & 0.05 & 0.80 & 0.12 & 19.11 & 0.19 & 17.54 & 0.39 \\
\hline 21 & UGC668 & 0.02 & 726 & 650 & 50 & 11.42 & 0.05 & 11.31 & 0.06 & 0.11 & 0.08 & 11.43 & 0.05 & 11.35 & 0.07 \\
\hline 22 & UGC685 & 0.06 & 60 & 45 & -60 & 16.04 & 0.05 & 15.66 & 0.03 & 0.38 & 0.06 & 16.03 & 0.05 & 15.61 & 0.03 \\
\hline 23 & UGC695 & 0.03 & 78 & 64 & -45 & 16.61 & 0.05 & 16.24 & 0.04 & 0.37 & 0.06 & 16.62 & 0.05 & 16.25 & 0.04 \\
\hline 24 & UGC891 & 0.03 & 102 & 44 & 47 & 16.31 & 0.05 & 16.08 & 0.03 & 0.23 & 0.06 & 16.31 & 0.05 & 16.07 & 0.03 \\
\hline 25 & UGC1056 & 0.07 & 78 & 52 & 45 & 16.63 & 0.05 & 16.25 & 0.03 & 0.38 & 0.06 & 16.64 & 0.05 & 16.25 & 0.03 \\
\hline 26 & UGC1104 & 0.06 & 78 & 47 & 5 & 15.60 & 0.05 & 15.41 & 0.03 & 0.19 & 0.06 & 15.60 & 0.05 & 15.40 & 0.03 \\
\hline 27 & NGC598 & 0.04 & 2070 & 1219 & 23 & 8.00 & 0.05 & 7.71 & 0.03 & 0.29 & 0.06 & 7.86 & 0.05 & 7.59 & 0.04 \\
\hline 28 & NGC625 & 0.02 & 342 & 112 & -88 & 13.72 & 0.05 & 13.34 & 0.03 & 0.38 & 0.06 & 13.76 & 0.05 & 13.39 & 0.03 \\
\hline 29 & NGC628 & 0.07 & 780 & 706 & 25 & 11.62 & 0.05 & 11.34 & 0.04 & 0.28 & 0.07 & 11.66 & 0.05 & 11.40 & 0.04 \\
\hline 30 & UGC1176 & 0.06 & 204 & 160 & 25 & 15.43 & 0.09 & 15.26 & & 0.17 & 0.14 & 15.43 & 0.12 & 15.22 & 0.13 \\
\hline 31 & UGCA20 & 0.06 & 228 & 59 & -27 & 16.33 & 0.09 & 16.24 & 0.09 & 0.09 & 0.13 & 16.33 & 0.11 & 16.18 & 0.10 \\
\hline 32 & ESO245-G005 & 0.02 & 318 & 283 & -58 & 13.62 & 0.05 & 13.55 & 0.04 & 0.07 & 0.06 & 13.63 & 0.05 & 13.58 & 0.04 \\
\hline 33 & UGC1249 & 0.08 & 306 & 137 & -30 & 13.44 & 0.05 & 13.27 & 0.03 & 0.17 & 0.06 & 13.44 & 0.05 & 13.27 & 0.03 \\
\hline 34 & NGC672 & 0.08 & 318 & 115 & 65 & 13.19 & 0.05 & 12.84 & 0.03 & 0.35 & 0.06 & 13.20 & 0.05 & 12.85 & 0.03 \\
\hline 35 & UGC1281 & 0.05 & 330 & 59 & 38 & 15.04 & 0.09 & 14.73 & 0.07 & 0.31 & 0.12 & 15.08 & 0.09 & 14.74 & 0.07 \\
\hline 36 & ESO245-G007 & 0.02 & 216 & 181 & 90 & 16.18 & 0.20 & 15.34 & 0.05 & 0.84 & 0.20 & 16.23 & 0.20 & 15.29 & 0.07 \\
\hline 37 & NGC784 & 0.06 & 294 & 67 & 0 & 13.93 & 0.05 & 13.49 & 0.03 & 0.44 & 0.06 & 13.92 & 0.05 & 13.48 & 0.03 \\
\hline 38 & UGC1561 & 0.08 & 72 & 65 & -80 & 16.34 & 0.09 & 16.07 & 0.04 & 0.27 & 0.10 & 16.36 & 0.09 & 16.06 & 0.05 \\
\hline 39 & NGC855 & 0.07 & 114 & 44 & 60 & 15.91 & 0.08 & 15.25 & 0.04 & 0.66 & 0.09 & 15.92 & 0.08 & 15.19 & 0.05 \\
\hline 40 & UGC1807 & 0.08 & 72 & 72 & 0 & 16.14 & 0.13 & 15.85 & 0.08 & 0.29 & 0.15 & 16.11 & 0.13 & 15.82 & 0.08 \\
\hline 41 & NGC891 & 0.06 & 600 & 111 & 22 & 14.61 & 0.19 & 13.80 & 0.06 & 0.81 & 0.20 & 14.61 & 0.19 & 13.82 & 0.06 \\
\hline 42 & UGC1865 & 0.07 & 114 & 86 & 80 & 16.08 & 0.08 & 15.69 & 0.05 & 0.39 & 0.09 & 16.04 & 0.11 & 15.66 & 0.06 \\
\hline 43 & NGC925 & 0.08 & 456 & 256 & -78 & 12.09 & 0.05 & 11.88 & 0.03 & 0.21 & 0.06 & 12.08 & 0.05 & 11.87 & 0.03 \\
\hline 44 & UGC1924 & 0.08 & 84 & 15 & 3 & 16.96 & 0.09 & 16.62 & 0.05 & 0.34 & 0.10 & 16.96 & 0.09 & 16.59 & 0.05 \\
\hline 45 & NGC949 & 0.06 & 102 & 55 & -35 & 14.98 & 0.08 & 14.38 & 0.04 & 0.60 & 0.09 & 14.98 & 0.08 & 14.37 & 0.04 \\
\hline 46 & NGC959 & 0.07 & 126 & 77 & 65 & 14.70 & 0.06 & 14.29 & 0.03 & 0.41 & 0.07 & 14.70 & 0.06 & 14.29 & 0.03 \\
\hline 47 & UGC2014 & 0.05 & 54 & 16 & -4 & 17.94 & 0.11 & 17.85 & 0.08 & 0.09 & 0.14 & 17.89 & 0.14 & 17.81 & 0.20 \\
\hline 48 & UGC2023 & 0.09 & 108 & 108 & 0 & 15.14 & 0.11 & 14.94 & 0.06 & 0.20 & 0.13 & 15.11 & 0.12 & 14.91 & 0.06 \\
\hline 49 & UGC2034 & 0.06 & $\ldots$ & $\ldots$ & $\ldots$ & $\ldots$ & $\ldots$ & $\ldots$ & $\ldots$ & $\ldots$ & $\ldots$ & $\ldots$ & $\ldots$ & $\ldots$ & $\ldots$ \\
\hline 50 & ESO115-G021 & 0.03 & 366 & 37 & 44 & 14.63 & 0.05 & 14.36 & 0.04 & 0.27 & 0.06 & 14.62 & 0.05 & 14.35 & 0.04 \\
\hline 51 & NGC1003 & 0.07 & 390 & 135 & -83 & 13.68 & 0.05 & 13.05 & 0.07 & 0.63 & 0.09 & 13.67 & 0.05 & 13.07 & 0.07 \\
\hline 52 & Maffei2 & 2.42 & $\ldots$ & $\ldots$ & $\ldots$ & $\ldots$ & $\ldots$ & $\ldots$ & $\ldots$ & $\ldots$ & $\ldots$ & $\ldots$ & $\ldots$ & $\ldots$ & $\ldots$ \\
\hline 53 & NGC1058 & 0.06 & 222 & 207 & -85 & 13.69 & 0.05 & 13.24 & 0.03 & 0.45 & 0.06 & 13.58 & 0.06 & 13.25 & 0.06 \\
\hline 54 & UGC2259 & 0.07 & $\ldots$ & $\ldots$ & $\ldots$ & $\ldots$ & $\ldots$ & $\ldots$ & $\ldots$ & $\ldots$ & $\ldots$ & $\ldots$ & $\ldots$ & $\ldots$ & $\ldots$ \\
\hline 55 & ESO154-G023 & 0.02 & 378 & 80 & 39 & 13.78 & 0.05 & 13.52 & 0.04 & 0.26 & 0.06 & 13.79 & 0.05 & 13.52 & 0.04 \\
\hline 56 & NGC1156 & 0.22 & 246 & 186 & 25 & 12.58 & 0.09 & 12.38 & 0.05 & 0.20 & 0.10 & 12.63 & 0.09 & 12.40 & 0.05 \\
\hline 57 & ESO300-G014 & 0.02 & 264 & 132 & -14 & 14.88 & 0.06 & 14.65 & 0.03 & 0.23 & 0.07 & 14.89 & 0.06 & 14.65 & 0.03 \\
\hline 58 & ESO300-G016 & 0.02 & 63 & 63 & 0 & & $\ldots$ & 17.06 & 0.04 & $\ldots$ & $\ldots$ & 17.29 & 0.05 & 17.06 & 0.05 \\
\hline 59 & NGC1291 & 0.01 & 438 & 362 & -15 & 14.23 & 0.10 & 13.39 & 0.05 & 0.84 & 0.11 & 14.19 & 0.10 & 13.34 & 0.05 \\
\hline 60 & NGC1313 & 0.11 & 516 & 391 & 40 & 10.59 & 0.08 & 10.43 & 0.04 & 0.16 & 0.09 & 10.58 & 0.08 & 10.42 & 0.05 \\
\hline 61 & NGC1311 & 0.02 & 216 & 58 & 40 & 14.84 & 0.05 & 14.41 & 0.03 & 0.43 & 0.06 & 14.85 & 0.05 & 14.42 & 0.03 \\
\hline 62 & UGC2684 & 0.14 & 144 & 72 & -60 & 16.45 & 0.19 & 16.66 & 0.07 & -0.21 & 0.20 & 16.67 & 0.22 & 16.70 & 0.07 \\
\hline 63 & UGC2689 & 0.15 & $\ldots$ & $\ldots$ & $\ldots$ & $\ldots$ & $\ldots$ & $\ldots$ & $\ldots$ & $\ldots$ & $\ldots$ & $\ldots$ & $\ldots$ & $\ldots$ & $\ldots$ \\
\hline
\end{tabular}


Table 2

(Continued)

\begin{tabular}{|c|c|c|c|c|c|c|c|c|c|c|c|c|c|c|c|}
\hline \multirow{2}{*}{$\begin{array}{l}\text { No. } \\
\text { (1) } \\
\end{array}$} & \multirow{2}{*}{$\begin{array}{c}\text { Galaxy Name } \\
\text { (2) }\end{array}$} & \multirow{2}{*}{$\begin{array}{c}E(B-V) \\
(3)\end{array}$} & \multirow{2}{*}{$\begin{array}{l}a \\
(") \\
(4)\end{array}$} & \multirow{2}{*}{$\begin{array}{l}b \\
\left({ }^{\prime \prime}\right) \\
(5)\end{array}$} & \multirow{2}{*}{$\begin{array}{l}\text { P.A. } \\
(\mathrm{deg}) \\
(6)\end{array}$} & \multicolumn{2}{|c|}{$\begin{array}{l}\mathrm{FUV}_{\mathrm{ap}} \\
(\mathrm{mag})\end{array}$} & \multicolumn{2}{|c|}{$\begin{array}{l}\mathrm{NUV}_{\text {ap }} \\
(\mathrm{mag})\end{array}$} & \multicolumn{2}{|c|}{$\begin{array}{c}(\mathrm{FUV}- \\
\mathrm{NUV})_{\mathrm{ap}} \\
(\mathrm{mag})\end{array}$} & $\begin{array}{l}\text { FUV } \\
(\mathrm{m}\end{array}$ & $\begin{array}{l}\text { asym } \\
\text { g) }\end{array}$ & NUV & asym \\
\hline & & & & & & (7) & (8) & (9) & (10) & (11) & (12) & (13) & (14) & (15) & (16) \\
\hline 64 & UGC2716 & 0.14 & 96 & 54 & 88 & 15.92 & 0.06 & 15.43 & 0.06 & 0.49 & 0.08 & 15.93 & 0.06 & 15.42 & 0.07 \\
\hline 65 & IC1959 & 0.01 & 204 & 51 & -33 & 14.37 & 0.05 & 14.24 & 0.03 & 0.13 & 0.06 & 14.37 & 0.05 & 14.25 & 0.03 \\
\hline 66 & UGC2847 & 0.56 & 996 & 973 & -28 & 8.48 & 0.13 & 8.31 & 0.27 & 0.17 & 0.30 & 8.16 & 0.13 & 8.15 & 0.27 \\
\hline 67 & IC2000 & 0.01 & 270 & 53 & 83 & 15.22 & 0.06 & 14.72 & 0.03 & 0.50 & 0.07 & 15.23 & 0.06 & 14.73 & 0.03 \\
\hline 68 & ESO302-G014 & 0.01 & 126 & 111 & -59 & 15.75 & 0.06 & 15.77 & 0.05 & -0.02 & 0.08 & 15.76 & 0.06 & 15.78 & 0.05 \\
\hline 69 & NGC1487 & 0.01 & 294 & 187 & 55 & 13.41 & 0.05 & 13.23 & 0.03 & 0.18 & 0.06 & 13.46 & 0.05 & 13.26 & 0.03 \\
\hline 70 & ESO249-G036 & 0.01 & 150 & 136 & -22 & 16.13 & 0.06 & 16.04 & 0.03 & 0.09 & 0.07 & 16.13 & 0.06 & 16.03 & 0.03 \\
\hline 71 & UGCA86 & 0.94 & 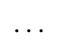 & & 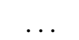 & $(14.30)$ & & (14.39) & & . & & & & & \\
\hline 72 & NGC1510 & 0.01 & 96 & 52 & 90 & 15.09 & 0.05 & 14.87 & 0.03 & 0.22 & 0.06 & 15.09 & 0.05 & 14.87 & 0.03 \\
\hline 73 & NGC1512 & 0.01 & 798 & 502 & 90 & 13.36 & 0.05 & 13.12 & 0.07 & 0.24 & 0.08 & 13.27 & 0.05 & 13.19 & 0.12 \\
\hline 74 & NGC1522 & 0.01 & 102 & 68 & 42 & 15.15 & 0.05 & 15.02 & 0.03 & 0.13 & 0.06 & 15.16 & 0.05 & 15.02 & 0.03 \\
\hline 75 & NGC1518 & 0.05 & 222 & 74 & 35 & 13.42 & 0.05 & 13.17 & 0.03 & 0.25 & 0.06 & 13.43 & 0.05 & 13.18 & 0.03 \\
\hline 76 & ESO483-G013 & 0.05 & 138 & 69 & -44 & 15.75 & 0.05 & 15.41 & 0.03 & 0.34 & 0.06 & 15.79 & 0.06 & 15.41 & 0.03 \\
\hline 77 & NGC1556 & 0.02 & 174 & 51 & -13 & 14.83 & 0.05 & 14.62 & 0.03 & 0.21 & 0.06 & 14.85 & 0.05 & 14.64 & 0.03 \\
\hline 78 & UGCA90 & 0.02 & 402 & 151 & -48 & 14.12 & 0.06 & 13.94 & 0.05 & 0.18 & 0.08 & 14.13 & 0.06 & 13.95 & 0.05 \\
\hline 79 & NGC1592 & 0.04 & 102 & 51 & -84 & 14.06 & 0.05 & 14.10 & 0.03 & -0.04 & 0.06 & 14.07 & 0.05 & 14.10 & 0.03 \\
\hline 80 & NGC1569 & 0.69 & 264 & 132 & -60 & 9.65 & 0.05 & 9.02 & 0.04 & 0.63 & 0.07 & 9.77 & 0.06 & 9.17 & 0.05 \\
\hline 81 & UGCA92 & 0.78 & . & . & . & (15.69) & & $(15.88)$ & & $\ldots$ & $\ldots$ & $\ldots$ & 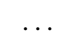 & $\ldots$ & .. \\
\hline 82 & NGC1560 & 0.19 & 396 & 68 & 23 & 13.30 & 0.05 & 12.89 & 0.03 & 0.41 & 0.06 & 13.17 & 0.06 & 12.77 & 0.04 \\
\hline 83 & ESO158-G003 & 0.01 & 114 & 107 & -10 & 15.47 & 0.05 & 15.20 & 0.03 & 0.27 & 0.06 & 15.48 & 0.05 & 15.20 & 0.03 \\
\hline 84 & UGC3174 & 0.09 & 78 & 50 & 85 & 16.12 & 0.18 & 15.76 & 0.05 & 0.36 & 0.18 & 16.01 & 0.38 & 15.56 & 0.12 \\
\hline 85 & ESO119-G016 & 0.02 & 102 & 44 & 25 & 16.05 & 0.05 & 15.77 & 0.03 & 0.28 & 0.06 & 16.01 & 0.07 & 15.73 & 0.04 \\
\hline 86 & NGC1705 & 0.01 & 138 & 102 & 50 & 13.33 & 0.05 & 13.41 & 0.03 & -0.08 & 0.06 & 13.34 & 0.05 & 13.41 & 0.03 \\
\hline 87 & ESO252-IG001 & 0.01 & $\ldots$ & $\ldots$ & $\ldots$ & $\ldots$ & $\ldots$ & $\ldots$ & $\ldots$ & $\ldots$ & $\ldots$ & $\ldots$ & $\ldots$ & $\ldots$ & $\ldots$ \\
\hline 88 & NGC1744 & 0.04 & 480 & 261 & -12 & 13.01 & 0.05 & 12.83 & 0.03 & 0.18 & 0.06 & 12.99 & 0.05 & 12.81 & 0.03 \\
\hline 89 & NGC1796 & 0.02 & 108 & 57 & -78 & 14.94 & 0.05 & 14.43 & 0.03 & 0.51 & 0.06 & 14.93 & 0.05 & 14.43 & 0.03 \\
\hline 90 & ESO486-G021 & 0.03 & 84 & 56 & -73 & 15.28 & 0.05 & 15.07 & 0.03 & 0.21 & 0.06 & 15.28 & 0.05 & 15.07 & 0.03 \\
\hline 91 & MCG-05-13-004 & 0.01 & $\ldots$ & $\ldots$ & $\ldots$ & $\ldots$ & $\ldots$ & $\ldots$ & $\ldots$ & $\ldots$ & $\ldots$ & $\ldots$ & $\ldots$ & $\ldots$ & \\
\hline 92 & NGC1800 & 0.01 & 144 & 79 & -67 & 14.59 & 0.05 & 14.43 & 0.03 & 0.16 & 0.06 & 14.61 & 0.05 & 14.43 & 0.03 \\
\hline 93 & NGC1808 & 0.03 & 396 & 238 & -47 & 14.20 & 0.10 & 13.51 & 0.06 & 0.69 & 0.12 & 14.03 & 0.12 & 13.43 & 0.07 \\
\hline 94 & ESO305-G009 & 0.03 & 240 & 192 & 63 & 14.31 & 0.07 & 14.15 & 0.04 & 0.16 & 0.08 & 14.30 & 0.07 & 14.15 & 0.04 \\
\hline 95 & UGCA103 & 0.01 & $\ldots$ & $\ldots$ & $\ldots$ & $\ldots$ & & $\ldots$ & $\ldots$ & $\ldots$ & $\ldots$ & & & $\ldots$ & \\
\hline 96 & UGC & 0.02 & 258 & 221 & 10 & 13.98 & 0.05 & 13.83 & 0.09 & 0.15 & 0.11 & 13.99 & 0.05 & 13.87 & 0.10 \\
\hline 97 & UGCA105 & 0.31 & 378 & 240 & 55 & 12.55 & 0.06 & 12.20 & 0.35 & 0.35 & 0.36 & 12.51 & 0.07 & 12.08 & 0.35 \\
\hline 98 & LMC & 0.07 & $\ldots$ & $\ldots$ & $\ldots$ & $\ldots$ & $\ldots$ & $\ldots$ & $\ldots$ & $\ldots$ & $\ldots$ & $\ldots$ & $\ldots$ & $\ldots$ & $\ldots$ \\
\hline 99 & UGC3303 & 0.13 & $\ldots$ & $\ldots$ & $\ldots$ & $\ldots$ & $\ldots$ & $\ldots$ & $\ldots$ & $\ldots$ & $\ldots$ & $\ldots$ & $\ldots$ & $\ldots$ & $\ldots$ \\
\hline 100 & ESO553-G046 & 0.05 & 78 & 52 & -13 & 15.11 & 0.05 & 15.00 & 0.05 & 0.11 & 0.07 & 15.11 & 0.05 & 15.01 & 0.05 \\
\hline 101 & ESO306-G013 & 0.04 & $\ldots$ & $\ldots$ & $\ldots$ & $\ldots$ & $\ldots$ & $\ldots$ & $\ldots$ & $\ldots$ & $\ldots$ & $\ldots$ & $\ldots$ & $\ldots$ & $\ldots$ \\
\hline 102 & UGCA114 & 0.16 & 126 & 92 & -10 & 13.75 & 0.07 & 13.40 & 0.08 & 0.35 & 0.10 & 13.72 & 0.08 & 13.36 & 0.08 \\
\hline 103 & UGCA116 & 0.82 & 42 & 14 & -25 & 12.72 & 0.10 & 12.17 & 0.05 & 0.55 & 0.11 & 12.49 & 0.12 & 12.02 & 0.15 \\
\hline 104 & KKH34 & 0.24 & $\ldots$ & $\ldots$ & $\ldots$ & (20.06) & $\ldots$ & $(20.32)$ & $\ldots$ & $\ldots$ & $\ldots$ & $\ldots$ & $\ldots$ & $\ldots$ & $\ldots$ \\
\hline 105 & ESO364-G?029 & 0.05 & $\ldots$ & $\ldots$ & $\ldots$ & $\ldots$ & $\ldots$ & $\ldots$ & .. & $\ldots$ & .. & $\ldots$ & . . & $\ldots$ & $\ldots$ \\
\hline 106 & AM0605-341 & 04 & $\ldots$ & $\ldots$ & $\ldots$ & $\ldots$ & $\ldots$ & $\ldots$ & $\ldots$ & $\ldots$ & $\ldots$ & $\ldots$ & $\ldots$ & $\ldots$ & $\ldots$ \\
\hline 107 & NGC2188 & 0.03 & $\ldots$ & $\ldots$ & $\ldots$ & $\ldots$ & $\ldots$ & $\ldots$ & $\ldots$ & $\ldots$ & $\ldots$ & $\ldots$ & $\ldots$ & $\ldots$ & $\ldots$ \\
\hline 108 & UGCA120 & 0.08 & $\ldots$ & $\ldots$ & $\ldots$ & $\ldots$ & $\ldots$ & $\ldots$ & $\ldots$ & $\ldots$ & $\ldots$ & $\ldots$ & $\ldots$ & $\ldots$ & $\ldots$ \\
\hline 109 & UGCA127 & 0.83 & $\ldots$ & $\ldots$ & $\ldots$ & $\ldots$ & $\ldots$ & $\ldots$ & $\ldots$ & $\ldots$ & $\ldots$ & $\ldots$ & $\ldots$ & $\ldots$ & $\ldots$ \\
\hline 110 & UGC 3475 & 0.18 & $\ldots$ & $\ldots$ & $\ldots$ & $\ldots$ & $\ldots$ & $\ldots$ & $\ldots$ & $\ldots$ & $\ldots$ & $\ldots$ & $\ldots$ & $\ldots$ & $\ldots$ \\
\hline 111 & ESO255-G019 & 0.08 & $\ldots$ & $\ldots$ & $\ldots$ & $\ldots$ & $\ldots$ & $\ldots$ & $\ldots$ & $\ldots$ & $\ldots$ & $\ldots$ & $\ldots$ & $\ldots$ & $\ldots$ \\
\hline 112 & KКH37 & 0.07 & 42 & 32 & 50 & 18.32 & 0.07 & 17.81 & 0.04 & 0.51 & 0.08 & 18.35 & 0.07 & 17.70 & 0.05 \\
\hline 113 & ESO207-G007 & 0.08 & 96 & 66 & 6 & 15.31 & 0.11 & 15.10 & 0.08 & 0.21 & 0.14 & 15.09 & 0.13 & 14.97 & 0.10 \\
\hline 114 & UGC3600 & 0.09 & 36 & 16 & 35 & 17.44 & 0.09 & 17.07 & 0.05 & 0.37 & 0.10 & 16.88 & 0.23 & 16.78 & 0.05 \\
\hline 115 & AM0704-582 & 0.12 & $\ldots$ & $\ldots$ & $\ldots$ & $\ldots$ & & $\ldots$ & $\ldots$ & $\ldots$ & $\ldots$ & & & & \\
\hline 116 & NGC2337 & 0.09 & 90 & 70 & -60 & 14.48 & 0.06 & 14.14 & 0.03 & 0.34 & 0.07 & 14.45 & 0.06 & 14.10 & 0.03 \\
\hline 117 & UGC3817 & 0.10 & 102 & 51 & 50 & 16.88 & 0.25 & 16.46 & 0.09 & 0.42 & 0.26 & 17.04 & 0.26 & 16.41 & 0.11 \\
\hline 118 & UGC3860 & 0.06 & 78 & 54 & 20 & 16.33 & 0.07 & 15.97 & 0.04 & 0.36 & 0.08 & 16.32 & 0.08 & 15.96 & 0.04 \\
\hline 119 & NGC2366 & 0.04 & 654 & 269 & 25 & 12.42 & 0.05 & 12.34 & 0.03 & 0.08 & 0.06 & 12.43 & 0.05 & 12.35 & 0.03 \\
\hline 120 & UGC3876 & 0.05 & $\ldots$ & $\ldots$ & $\ldots$ & $\ldots$ & $\ldots$ & $\ldots$ & $\ldots$ & $\ldots$ & $\ldots$ & $\ldots$ & $\ldots$ & $\ldots$ & $\ldots$ \\
\hline 121 & ESO059-G001 & 0.15 & $\ldots$ & $\ldots$ & $\ldots$ & $\ldots$ & $\ldots$ & $\ldots$ & $\ldots$ & $\ldots$ & $\ldots$ & $\ldots$ & $\ldots$ & $\ldots$ & $\ldots$ \\
\hline 122 & NGC2427 & 0.21 & $\ldots$ & $\ldots$ & $\ldots$ & $\ldots$ & $\ldots$ & $\ldots$ & $\ldots$ & $\ldots$ & $\ldots$ & $\ldots$ & $\ldots$ & $\ldots$ & $\ldots$ \\
\hline 123 & NGC2403 & 0.04 & 960 & 540 & -53 & 10.31 & 0.05 & 10.12 & 0.04 & 0.19 & 0.06 & 10.31 & 0.05 & 10.12 & 0.04 \\
\hline 124 & UGC3966 & 0.05 & 114 & 114 & 0 & 15.83 & 0.06 & 15.60 & 0.05 & 0.23 & 0.08 & 15.82 & 0.06 & 15.60 & 0.05 \\
\hline 125 & UGC3974 & 0.03 & 228 & 221 & 0 & 14.77 & 0.05 & 14.46 & 0.03 & 0.31 & 0.06 & 14.77 & 0.05 & 14.45 & 0.03 \\
\hline
\end{tabular}


Table 2

(Continued)

\begin{tabular}{|c|c|c|c|c|c|c|c|c|c|c|c|c|c|c|c|}
\hline \multirow{2}{*}{$\begin{array}{l}\text { No. } \\
\text { (1) }\end{array}$} & \multirow{2}{*}{$\begin{array}{c}\text { Galaxy Name } \\
\text { (2) }\end{array}$} & \multirow{2}{*}{$\begin{array}{c}E(B-V) \\
(3)\end{array}$} & \multirow{2}{*}{$\begin{array}{l}a \\
(") \\
(4)\end{array}$} & \multirow{2}{*}{$\begin{array}{l}b \\
(") \\
(5)\end{array}$} & \multirow{2}{*}{$\begin{array}{l}\text { P.A. } \\
\text { (deg) } \\
(6)\end{array}$} & \multicolumn{2}{|c|}{$\begin{array}{l}\mathrm{FUV}_{\text {ap }} \\
(\mathrm{mag})\end{array}$} & \multicolumn{2}{|c|}{$\begin{array}{c}\mathrm{NUV}_{\text {ap }} \\
(\mathrm{mag})\end{array}$} & \multicolumn{2}{|c|}{$\begin{array}{c}(\mathrm{FUV}- \\
\mathrm{NUV})_{\mathrm{ap}} \\
(\mathrm{mag})\end{array}$} & $\begin{array}{r}\mathrm{FU} \\
(\mathrm{m}\end{array}$ & $\begin{array}{l}\text { sym } \\
\text { g) }\end{array}$ & NUY & \\
\hline & & & & & & (7) & (8) & (9) & $(10)$ & (11) & $(12)$ & $(13)$ & (14) & (15) & $(16)$ \\
\hline 126 & CGCG262-028 & 0.07 & 48 & 21 & -60 & 15.46 & 0.06 & 15.39 & 0.04 & 0.07 & 0.07 & 15.45 & 0.07 & 15.37 & 0.05 \\
\hline 127 & UGC4115 & 0.03 & 90 & 50 & -35 & 15.60 & 0.05 & 15.37 & 0.04 & 0.23 & 0.07 & 15.59 & 0.05 & 15.37 & 0.05 \\
\hline 128 & NGC2500 & 0.04 & 210 & 188 & 0 & 13.46 & 0.06 & 13.32 & 0.03 & 0.14 & 0.07 & 13.47 & 0.06 & 13.33 & 0.03 \\
\hline 129 & NGC2537 & 0.05 & 126 & 111 & 0 & 13.88 & 0.05 & 13.69 & 0.03 & 0.19 & 0.06 & 13.88 & 0.05 & 13.70 & 0.03 \\
\hline 130 & UGC4278 & 0.05 & 342 & 36 & -8 & 14.43 & 0.05 & 14.20 & 0.04 & 0.23 & 0.07 & 14.43 & 0.05 & 14.21 & 0.04 \\
\hline 131 & UGC4305 & 0.03 & 354 & 282 & 15 & 12.32 & 0.05 & 12.22 & 0.03 & 0.10 & 0.06 & 12.28 & 0.06 & 12.17 & 0.04 \\
\hline 132 & NGC2552 & 0.05 & 156 & 102 & 45 & 14.27 & 0.05 & 14.09 & 0.03 & 0.18 & 0.06 & 14.26 & 0.05 & 14.08 & 0.03 \\
\hline 133 & M81dwA & 0.02 & 72 & 39 & 35 & 17.30 & 0.05 & 17.29 & 0.03 & 0.01 & 0.06 & 17.28 & 0.05 & 17.25 & 0.03 \\
\hline 134 & SDSSJ0825+3532 & 0.05 & 12 & 6 & -40 & 18.35 & 0.06 & 18.32 & 0.03 & 0.03 & 0.07 & $\ldots$ & $\ldots$ & 18.21 & 0.03 \\
\hline 135 & UGC4426 & 0.04 & 144 & 72 & 10 & 16.72 & 0.06 & 16.48 & 0.03 & 0.24 & 0.07 & 16.70 & 0.06 & 16.44 & 0.04 \\
\hline 136 & UGC4459 & 0.04 & 108 & 94 & -60 & 15.27 & 0.05 & 15.30 & 0.04 & -0.03 & 0.06 & 15.27 & 0.05 & 15.29 & 0.04 \\
\hline 137 & ESO495-G021 & 0.11 & $\ldots$ & $\ldots$ & $\ldots$ & $\ldots$ & $\ldots$ & $\ldots$ & $\ldots$ & $\ldots$ & $\ldots$ & $\ldots$ & $\ldots$ & $\ldots$ & $\ldots$ \\
\hline 138 & UGC4483 & 0.03 & 78 & 57 & -18 & 15.71 & 0.05 & 15.75 & 0.03 & -0.04 & 0.06 & 15.71 & 0.05 & 15.76 & 0.03 \\
\hline 139 & NGC2683 & 0.03 & 414 & 98 & 44 & 14.14 & 0.05 & 13.52 & 0.05 & 0.62 & 0.07 & 14.13 & 0.05 & 13.50 & 0.06 \\
\hline 140 & UGC4704 & 0.03 & 306 & 30 & -65 & 15.85 & 0.07 & 15.62 & 0.04 & 0.23 & & 15.86 & 0.07 & 15.62 & 0.04 \\
\hline 141 & LSBCD564-08 & 0.03 & 42 & 30 & 0 & 18.99 & 0.07 & 18.57 & 0.05 & 0.42 & 0.09 & 18.76 & 0.13 & 18.38 & 0.05 \\
\hline 142 & UGC4787 & 0.02 & 144 & 41 & 6 & 15.99 & 0.05 & 15.57 & 0.04 & 0.42 & 0.06 & 16.00 & 0.05 & 15.58 & 0.04 \\
\hline 143 & LSBCD634-03 & 0.04 & $\ldots$ & $\ldots$ & $\ldots$ & (21.85) & $\ldots$ & $(21.71)$ & $\ldots$ & $\ldots$ & $\ldots$ & $\ldots$ & $\ldots$ & $\ldots$ & $\ldots$ \\
\hline 144 & UGCA148 & 0.17 & $\ldots$ & $\ldots$ & $\ldots$ & $\ldots$ & $\ldots$ & $\ldots$ & $\ldots$ & $\ldots$ & $\ldots$ & $\ldots$ & $\ldots$ & $\ldots$ & $\ldots$ \\
\hline 145 & NGC2784 & 0.21 & 246 & 98 & 73 & 15.49 & 0.37 & 14.56 & 0.04 & 0.93 & 0.38 & 14.94 & 0.66 & 14.38 & 0.05 \\
\hline 146 & UGCA153 & 0.09 & 126 & 82 & -40 & 16.33 & 0.13 & 16.01 & 0.07 & 0.32 & 0.15 & 16.34 & 0.16 & 15.92 & 0.09 \\
\hline 147 & NGC2835 & 0.10 & 264 & 176 & 8 & 12.35 & 0.05 & 11.97 & 0.04 & 0.38 & 0.06 & 12.35 & 0.05 & 11.96 & 0.04 \\
\hline 148 & LSBCD565-06 & 0.04 & 36 & 31 & 0 & (21.77) & & 19.23 & 0.24 & $\ldots$ & $\ldots$ & $\ldots$ & $\ldots$ & 19.19 & 0.43 \\
\hline 149 & UGCA162 & 0.07 & 147 & 35 & 27 & $\ldots$ & $\ldots$ & 15.76 & 0.09 & $\ldots$ & $\ldots$ & 16.12 & 0.06 & 15.55 & 0.29 \\
\hline 150 & UGC4998 & 0.06 & 54 & 27 & 80 & 17.99 & 0.06 & 17.35 & 0.04 & 0.64 & 0.07 & 17.96 & 0.06 & 17.24 & 0.05 \\
\hline 151 & NGC2915 & 0.27 & 138 & 73 & -51 & 13.31 & 0.15 & 13.28 & 0.03 & 0.03 & 0.15 & 13.35 & 0.15 & 13.27 & 0.03 \\
\hline 152 & NGC2903 & 0.03 & 564 & 268 & 17 & 12.29 & 0.05 & 11.74 & 0.03 & 0.55 & 0.06 & 12.36 & 0.05 & 11.76 & 0.03 \\
\hline 153 & UGC5 & & $\ldots$ & $\ldots$ & $\ldots$ & & & & & & & $\ldots$ & $\ldots$ & $\ldots$ & $\ldots$ \\
\hline 154 & CGCG035-007 & 0.04 & 48 & 36 & 80 & 17.14 & 0.05 & 16.74 & 0.03 & 0.40 & 0.06 & 17.14 & 0.05 & 16.72 & 0.03 \\
\hline 155 & LeoT & 0.03 & 102 & 102 & 0 & 18.85 & 0.88 & 18.61 & 3.45 & 0.24 & 3.56 & 19.28 & 0.96 & 18.24 & 3.48 \\
\hline 156 & UGC5151 & 0.02 & $\ldots$ & $\ldots$ & & & & $\ldots$ & $\ldots$ & $\ldots$ & $\ldots$ & & $\ldots$ & & $\ldots$ \\
\hline 157 & UGC5139 & 0.05 & 246 & 205 & 0 & 14.60 & 0.09 & 14.50 & 0.05 & 0.10 & 0.10 & 14.59 & 0.09 & 14.50 & 0.05 \\
\hline 158 & IC559 & 0.03 & 66 & 50 & 90 & 16.45 & 0.05 & 16.08 & 0.03 & 0.37 & 0.06 & 16.45 & 0.05 & 16.04 & 0.03 \\
\hline 159 & UGC5209 & 0.02 & 30 & 30 & 0 & 18.10 & 0.09 & 17.68 & 0.05 & 0.42 & 0.10 & 18.08 & 0.10 & 17.61 & 0.05 \\
\hline 160 & NGC2976 & 0.07 & 264 & 121 & -37 & 13.17 & 0.05 & 12.69 & 0.03 & 0.48 & 0.06 & 13.19 & 0.05 & 12.71 & 0.03 \\
\hline 161 & UGC5272b & 0.02 & 30 & 15 & -65 & 18.40 & 0.06 & 18.35 & 0.04 & 0.05 & 0.07 & 18.41 & 0.07 & 18.35 & 0.04 \\
\hline 162 & UGC5272 & 0.02 & 144 & 55 & -65 & 14.94 & 0.05 & 14.87 & 0.03 & 0.07 & 0.06 & 14.94 & 0.05 & 14.87 & 0.03 \\
\hline 163 & UGC5288 & 0.03 & 96 & 59 & -25 & 15.73 & 0.05 & 15.52 & 0.03 & 0.21 & 0.06 & 15.70 & 0.05 & 15.48 & 0.03 \\
\hline 164 & NGC3037 & 0.09 & 48 & 44 & 44 & 15.16 & & 14.57 & 0.03 & 0.59 & 0.06 & 15.15 & 0.06 & 14.55 & 0.03 \\
\hline 165 & BK3N & 0.08 & 18 & 14 & 0 & 18.92 & & 18.88 & 0.04 & 0.04 & 0.07 & 18.77 & 0.09 & 18.72 & 0.05 \\
\hline 166 & NGC3031 & 0.08 & 1104 & 578 & -23 & 10.75 & 0.05 & 10.44 & 0.03 & 0.31 & 0.06 & 10.76 & 0.05 & 10.42 & 0.03 \\
\hline 167 & NGC3034 & 0.16 & 834 & 320 & 65 & 12.50 & 0.19 & 11.44 & 0.03 & 1.06 & 0.19 & 12.58 & 0.19 & 11.47 & 0.03 \\
\hline 168 & UGC5340 & 0.02 & 192 & 71 & 0 & 15.07 & 0.05 & 15.10 & 0.03 & -0.03 & 0.06 & 15.07 & 0.05 & 15.10 & 0.03 \\
\hline 169 & KDG61 & 0.07 & 30 & 25 & 40 & 22.23 & 0.33 & 19.65 & 0.04 & 2.58 & 0.34 & 21.81 & 0.58 & 19.09 & 0.21 \\
\hline 170 & UGC5336 & 0.08 & 168 & 134 & 40 & 14.95 & 0.08 & 14.80 & 0.09 & 0.15 & 0.12 & 14.95 & 0.08 & 14.80 & 0.09 \\
\hline 171 & ESO435-G016 & 0.10 & 126 & 82 & -61 & 15.45 & 0.09 & 14.76 & 0.03 & 0.69 & 0.09 & 15.54 & 0.10 & 14.74 & 0.03 \\
\hline 172 & ESO435-IG020 & 0.09 & 90 & 68 & -80 & 14.89 & 0.05 & 14.51 & 0.04 & 0.38 & 0.06 & 14.90 & 0.05 & 14.52 & 0.04 \\
\hline 173 & UGC5364 & 0.02 & 228 & 139 & -70 & 14.55 & 0.05 & 14.24 & 0.03 & 0.31 & 0.06 & 14.53 & 0.06 & 14.21 & 0.04 \\
\hline 174 & UGC5373 & 0.03 & 228 & 156 & -70 & 13.68 & 0.05 & 13.46 & 0.05 & 0.22 & 0.07 & 13.65 & 0.06 & 13.44 & 0.05 \\
\hline 175 & UGCA193 & & 162 & 12 & 17 & 16.36 & & 16.05 & 0.03 & 0.31 & 0.06 & 16.28 & 0.05 & 15.96 & 0.03 \\
\hline 176 & NGC3109 & 0.07 & 1074 & 208 & -87 & 11.37 & 0.19 & 11.09 & 0.10 & 0.28 & 0.21 & 11.42 & 0.19 & 11.14 & 0.10 \\
\hline 177 & NGC3077 & 0.07 & $\ldots$ & $\ldots$ & $\ldots$ & $\ldots$ & $\ldots$ & $\ldots$ & $\ldots$ & $\ldots$ & $\ldots$ & $\ldots$ & $\ldots$ & $\ldots$ & $\ldots$ \\
\hline 178 & UGCA196 & 0.08 & 204 & 83 & 53 & 14.52 & 0.09 & 14.15 & 0.08 & 0.37 & 0.11 & 14.51 & 0.09 & 14.13 & 0.08 \\
\hline 179 & NGC3113 & 0.10 & $\ldots$ & $\ldots$ & $\ldots$ & $\ldots$ & $\ldots$ & $\ldots$ & $\ldots$ & $\ldots$ & $\ldots$ & $\ldots$ & $\ldots$ & $\ldots$ & $\ldots$ \\
\hline 180 & UGC5427 & 0.02 & 84 & 56 & -60 & 16.20 & 0.05 & 15.96 & 0.03 & 0.24 & 0.06 & 16.21 & 0.05 & 15.96 & 0.03 \\
\hline 181 & UGC5428 & 0.10 & 108 & 108 & 0 & (22.67) & $\ldots$ & 17.82 & 0.09 & $\ldots$ & $\ldots$ & $\ldots$ & $\ldots$ & 17.66 & 0.16 \\
\hline 182 & NGC3115 & 0.05 & 510 & 177 & 40 & 15.75 & 0.21 & 14.11 & 0.09 & 1.64 & 0.23 & 15.70 & 0.21 & 14.15 & 0.10 \\
\hline 183 & UGC5423 & 0.08 & 66 & 44 & -40 & 16.58 & 0.07 & 16.38 & 0.04 & 0.20 & 0.08 & 16.57 & 0.07 & 16.35 & 0.05 \\
\hline 184 & NGC3125 & 0.08 & 126 & 80 & -66 & 14.37 & 0.08 & 13.99 & 0.04 & 0.38 & 0.09 & 14.40 & 0.08 & 14.02 & 0.04 \\
\hline 185 & UGC5453 & 0.03 & $\ldots$ & .. & $\ldots$ & $\ldots$ & $\ldots$ & $\ldots$ & $\ldots$ & $\ldots$ & & $\ldots$ & $\ldots$ & & $\ldots$ \\
\hline 186 & UGC5451 & 0.01 & 78 & 36 & -77 & 16.04 & 0.06 & 15.66 & 0.04 & 0.38 & 0.07 & 16.05 & 0.06 & 15.64 & 0.04 \\
\hline 187 & UGC5456 & 0.04 & 90 & 45 & -32 & 14.96 & 0.05 & 14.62 & 0.03 & 0.34 & 0.06 & 14.96 & 0.05 & 14.62 & 0.03 \\
\hline
\end{tabular}


Table 2

(Continued)

\begin{tabular}{|c|c|c|c|c|c|c|c|c|c|c|c|c|c|c|c|}
\hline \multirow{2}{*}{$\begin{array}{l}\text { No. } \\
\text { (1) }\end{array}$} & \multirow{2}{*}{$\begin{array}{c}\text { Galaxy Name } \\
\text { (2) }\end{array}$} & \multirow{2}{*}{$\begin{array}{c}E(B-V) \\
(3)\end{array}$} & \multirow{2}{*}{$\begin{array}{l}a \\
(") \\
(4) \\
\end{array}$} & \multirow{2}{*}{$\begin{array}{l}b \\
\\
(") \\
(5)\end{array}$} & \multirow{2}{*}{$\begin{array}{l}\text { P.A. } \\
(\mathrm{deg}) \\
(6)\end{array}$} & \multicolumn{2}{|c|}{$\begin{array}{l}\mathrm{FUV}_{\text {ap }} \\
(\mathrm{mag})\end{array}$} & \multicolumn{2}{|c|}{$\begin{array}{c}\mathrm{NUV}_{\text {ap }} \\
(\mathrm{mag})\end{array}$} & \multicolumn{2}{|c|}{$\begin{array}{c}\text { (FUV - } \\
\text { NUV })_{\text {ap }} \\
(\mathrm{mag})\end{array}$} & $\begin{array}{l}\text { FUV } \\
(\mathrm{m}\end{array}$ & $\begin{array}{l}\text { gisym } \\
\text { g) }\end{array}$ & $(\mathrm{m}$ & \\
\hline & & & & & & (7) & $(8)$ & (9) & $(10)$ & (11) & $(12)$ & (13) & $(14)$ & $(15)$ & (16) \\
\hline 188 & KUG1004+392 & 0.01 & 54 & 27 & -5 & 16.37 & 0.06 & 16.27 & 0.04 & 0.10 & 0.07 & 16.31 & 0.06 & 16.24 & 0.04 \\
\hline 189 & NGC3137 & 0.07 & $\ldots$ & $\ldots$ & $\ldots$ & $\ldots$ & $\ldots$ & $\ldots$ & $\ldots$ & $\ldots$ & $\ldots$ & $\ldots$ & $\ldots$ & $\ldots$ & $\ldots$ \\
\hline 190 & SextansA & 0.04 & 264 & 219 & 0 & 12.55 & 0.05 & 12.52 & 0.03 & 0.03 & 0.06 & 12.54 & 0.05 & 12.51 & 0.03 \\
\hline 191 & NGC3175 & 0.07 & 168 & 44 & 56 & 16.06 & 0.07 & 15.02 & 0.04 & 1.04 & 0.08 & 16.06 & 0.08 & 14.98 & 0.04 \\
\hline 192 & NGC3239 & 0.03 & 222 & 146 & 80 & 12.80 & 0.05 & 12.58 & 0.03 & 0.22 & 0.06 & 12.80 & 0.05 & 12.58 & 0.03 \\
\hline 193 & UGC5672 & 0.02 & 78 & 22 & -22 & 17.20 & 0.05 & 16.60 & 0.03 & 0.60 & 0.06 & 17.19 & 0.05 & 16.47 & 0.04 \\
\hline 194 & UGC5666 & 0.04 & 588 & 240 & 50 & 12.13 & 0.06 & 12.09 & 0.04 & 0.04 & 0.07 & 12.13 & 0.06 & 12.09 & 0.04 \\
\hline 195 & UGC5692 & 0.04 & 114 & 64 & 0 & 16.42 & 0.05 & 15.76 & 0.03 & 0.66 & 0.06 & 16.42 & 0.05 & 15.60 & 0.03 \\
\hline 196 & NGC3274 & 0.02 & 246 & 117 & -80 & 14.23 & 0.06 & 14.02 & 0.03 & 0.21 & 0.07 & 14.28 & 0.06 & 14.06 & 0.03 \\
\hline 197 & UGC5740 & 0.02 & 108 & 76 & -40 & 15.77 & 0.06 & 15.60 & 0.04 & 0.17 & 0.07 & 15.93 & 0.07 & 15.73 & 0.09 \\
\hline 198 & UGCA212 & 0.06 & 162 & 130 & 14 & 14.25 & 0.06 & 13.96 & 0.04 & 0.29 & 0.07 & 14.20 & 0.09 & 13.93 & 0.07 \\
\hline 199 & NGC3299 & 0.02 & 72 & 56 & 3 & 16.27 & 0.05 & 15.72 & 0.04 & 0.55 & 0.06 & 16.26 & 0.06 & 15.66 & 0.04 \\
\hline 200 & UGC5764 & 0.02 & 84 & 46 & 60 & 16.24 & 0.05 & 16.15 & 0.04 & 0.09 & 0.06 & 16.24 & 0.05 & 16.15 & 0.04 \\
\hline 201 & UGC5797 & 0.03 & 78 & 70 & -45 & 16.85 & 0.05 & 16.42 & 0.03 & 0.43 & 0.06 & 16.85 & 0.05 & 16.40 & 0.03 \\
\hline 202 & IC625 & 0.07 & 126 & 27 & -75 & 15.13 & 0.06 & 14.70 & 0.04 & 0.43 & 0.07 & 15.10 & 0.06 & 14.67 & 0.04 \\
\hline 203 & UGC5829 & 0.02 & 210 & 188 & 55 & 13.96 & 0.05 & 13.89 & 0.03 & 0.07 & 0.06 & 13.98 & 0.06 & 13.89 & 0.04 \\
\hline 204 & NGC3344 & 0.03 & 504 & 461 & 0 & 12.36 & 0.05 & 12.06 & 0.03 & 0.30 & 0.06 & 12.36 & 0.05 & 12.06 & 0.03 \\
\hline 205 & NGC3351 & 0.03 & 330 & 223 & 13 & 13.27 & 0.05 & 12.72 & 0.03 & 0.55 & 0.06 & 13.26 & 0.05 & 12.71 & 0.03 \\
\hline 206 & NGC3365 & 0.05 & 336 & 60 & -21 & 15.30 & 0.10 & 14.84 & 0.03 & 0.46 & 0.11 & 15.32 & 0.10 & 14.86 & 0.03 \\
\hline 207 & NGC3368 & 0.03 & 336 & 230 & 5 & 14.00 & 0.07 & 13.36 & 0.04 & 0.64 & 0.08 & 13.99 & 0.07 & 13.34 & 0.04 \\
\hline 208 & UGC5889 & 0.03 & 96 & 92 & 30 & 16.30 & 0.05 & 15.87 & 0.05 & 0.43 & 0.07 & 16.30 & 0.05 & 15.87 & 0.05 \\
\hline 209 & UGC5917 & 0.02 & 54 & 29 & -17 & 16.55 & 0.06 & 16.14 & 0.04 & 0.41 & 0.07 & 16.54 & 0.08 & 16.13 & 0.04 \\
\hline 210 & UGC5923 & 0.03 & 48 & 21 & -7 & 17.27 & 0.05 & 16.74 & 0.03 & 0.53 & 0.06 & 17.26 & 0.05 & 16.70 & 0.03 \\
\hline 211 & UGC5918 & 0.01 & 120 & 120 & 0 & 16.41 & 0.31 & 16.16 & 0.09 & 0.25 & 0.33 & 16.38 & 0.31 & 16.13 & 0.09 \\
\hline 212 & NGC3412 & 0.03 & 90 & 50 & -25 & 18.21 & 0.16 & 15.85 & 0.04 & 2.36 & 0.16 & 18.21 & 0.16 & 15.70 & 0.04 \\
\hline 213 & ESO376-G022 & 0.09 & $\ldots$ & ... & $\ldots$ & $\ldots$ & $\ldots$ & $\ldots$ & $\ldots$ & $\ldots$ & $\ldots$ & $\ldots$ & $\ldots$ & $\ldots$ & $\ldots$ \\
\hline 214 & NGC3432 & 0.01 & 300 & 66 & 38 & 13.27 & 0.05 & 12.84 & 0.03 & 0.43 & 0.06 & 13.26 & 0.05 & 12.84 & 0.03 \\
\hline 215 & KDG73 & 0.02 & 30 & 20 & -60 & 18.93 & 0.06 & 18.71 & 0.04 & 0.22 & 0.08 & 18.81 & 0.08 & 18.51 & 0.06 \\
\hline 216 & NGC3489 & 0.02 & 258 & 147 & 70 & 16.54 & 0.08 & 14.81 & 0.03 & 1.73 & 0.08 & 16.60 & 0.08 & 14.81 & 0.03 \\
\hline 217 & NGC3486 & 0.02 & 318 & 233 & 80 & 12.54 & 0.05 & 12.30 & 0.03 & 0.24 & 0.06 & 12.52 & 0.05 & 12.27 & 0.04 \\
\hline 218 & UGC6102 & 0.03 & 66 & 53 & -40 & 16.53 & 0.06 & 16.41 & 0.04 & 0.12 & 0.07 & 16.51 & 0.06 & 16.41 & 0.04 \\
\hline 219 & NGC3510 & 0.03 & 174 & 35 & -17 & 14.63 & 0.05 & 14.35 & 0.03 & 0.28 & 0.06 & 14.63 & 0.05 & 14.34 & 0.03 \\
\hline 220 & ESO377-G003 & 0.06 & 48 & 19 & 48 & 15.94 & 0.06 & 15.69 & 0.04 & 0.25 & 0.07 & 15.90 & 0.07 & 15.67 & 0.04 \\
\hline 221 & MRK36 & 0.03 & 60 & 40 & -45 & 15.64 & 0.05 & 15.62 & 0.03 & 0.02 & 0.06 & 15.64 & 0.05 & 15.61 & 0.03 \\
\hline 222 & NGC3521 & 0.06 & 492 & 228 & -17 & 13.02 & 0.06 & 12.26 & 0.04 & 0.76 & 0.07 & 13.05 & 0.07 & 12.27 & 0.04 \\
\hline 223 & UGC6161 & 0.01 & 156 & 72 & 40 & 15.37 & 0.05 & 15.18 & 0.03 & 0.19 & 0.06 & 15.36 & 0.05 & 15.18 & 0.03 \\
\hline 224 & MESSIER108 & 0.02 & 390 & 99 & 80 & 13.34 & 0.05 & 12.85 & 0.03 & 0.49 & 0.06 & 13.35 & 0.05 & 12.86 & 0.03 \\
\hline 225 & NGC3593 & 0.02 & 126 & 92 & -88 & 16.79 & 0.16 & 15.31 & 0.05 & 1.48 & 0.17 & 16.86 & 0.17 & 15.21 & 0.07 \\
\hline 226 & NGC3599 & 0.02 & 96 & 75 & -75 & 18.19 & 0.06 & 16.64 & 0.04 & 1.55 & 0.07 & 18.14 & 0.07 & 16.55 & 0.04 \\
\hline 227 & NGC3600 & 0.02 & 306 & 67 & 3 & 15.16 & 0.05 & 14.77 & 0.07 & 0.39 & 0.09 & 15.15 & 0.05 & 14.79 & 0.07 \\
\hline 228 & NGC3621 & 0.08 & 696 & 402 & -21 & 11.62 & 0.05 & 11.18 & 0.05 & 0.44 & 0.07 & 11.60 & 0.05 & 11.03 & 0.08 \\
\hline 229 & NGC3623 & 0.02 & 438 & 130 & -6 & 14.98 & 0.08 & 13.93 & 0.07 & 1.05 & 0.10 & 15.01 & 0.08 & 13.96 & 0.07 \\
\hline 230 & NGC3627 & 0.03 & 408 & 188 & -7 & 12.65 & 0.05 & 11.98 & 0.03 & 0.67 & 0.06 & 12.67 & 0.05 & 12.00 & 0.03 \\
\hline 231 & NGC3628 & 0.03 & 660 & 134 & -76 & 14.36 & 0.06 & 13.32 & 0.03 & 1.04 & 0.07 & 14.33 & 0.06 & 13.26 & 0.03 \\
\hline 232 & IC2782 & 0.02 & 78 & 64 & 40 & (21.93) & $\ldots$ & 17.89 & 0.08 & $\ldots$ & $\ldots$ & $\ldots$ & $\ldots$ & 18.31 & 0.18 \\
\hline 233 & IC2787 & 0.03 & $\ldots$ & $\ldots$ & $\ldots$ & $\ldots$ & $\ldots$ & $\ldots$ & $\ldots$ & $\ldots$ & $\ldots$ & $\ldots$ & $\ldots$ & $\ldots$ & $\ldots$ \\
\hline 234 & NGC3675 & 0.02 & 240 & 126 & -2 & 15.02 & 0.06 & 14.18 & 0.07 & 0.84 & 0.09 & 14.99 & 0.06 & 14.19 & 0.07 \\
\hline 235 & UGC6457 & 0.03 & 90 & 70 & -30 & 16.33 & 0.06 & 15.95 & 0.03 & 0.38 & 0.07 & 16.32 & 0.06 & 15.95 & 0.03 \\
\hline 236 & UGC6456 & 0.04 & 102 & 58 & -10 & 15.00 & 0.05 & 14.98 & 0.04 & 0.02 & 0.06 & 15.00 & 0.05 & 14.98 & 0.04 \\
\hline 237 & UGC6541 & 0.02 & 84 & 42 & -47 & 15.36 & 0.05 & 15.17 & 0.03 & 0.19 & 0.06 & 15.35 & 0.05 & 15.15 & 0.03 \\
\hline 238 & NGC3738 & 0.01 & 108 & 82 & -25 & 13.77 & 0.05 & 13.45 & 0.03 & 0.32 & 0.06 & 13.77 & 0.05 & 13.45 & 0.03 \\
\hline 239 & NGC3741 & 0.02 & 168 & 92 & 5 & 15.13 & 0.05 & 15.00 & 0.03 & 0.13 & 0.06 & 15.14 & 0.05 & 15.00 & 0.03 \\
\hline 240 & LEDA166115 & 0.02 & 24 & 16 & 0 & (22.03) & $\ldots$ & 19.61 & 0.17 & $\ldots$ & $\ldots$ & $\ldots$ & $\ldots$ & 19.41 & 0.18 \\
\hline 241 & UGC6817 & 0.03 & 306 & 112 & 65 & 14.78 & 0.05 & 14.57 & 0.06 & 0.21 & 0.08 & 14.78 & 0.05 & 14.58 & 0.06 \\
\hline 242 & UGC6900 & 0.02 & 90 & 56 & -65 & 17.42 & 0.05 & 16.91 & 0.04 & 0.51 & 0.07 & $\ldots$ & $\ldots$ & 16.86 & 0.05 \\
\hline 243 & BTS76 & 0.02 & 12 & 8 & 45 & 20.39 & 0.09 & 19.86 & 0.05 & 0.53 & 0.10 & 20.37 & 0.12 & 19.86 & 0.06 \\
\hline 244 & NGC4020 & 0.02 & 156 & 67 & 15 & 15.20 & 0.05 & 14.82 & 0.03 & 0.38 & 0.06 & 15.20 & 0.05 & 14.83 & 0.03 \\
\hline 245 & UGC7007 & 0.02 & 60 & 56 & -30 & 16.98 & 0.08 & 16.77 & 0.05 & 0.21 & 0.09 & 16.91 & 0.09 & 16.51 & 0.07 \\
\hline 246 & NGC4068 & 0.02 & 144 & 74 & 30 & 14.30 & 0.05 & 14.08 & 0.03 & 0.22 & 0.06 & 14.29 & 0.05 & 14.07 & 0.03 \\
\hline 247 & NGC4080 & 0.03 & 114 & 48 & -55 & 16.08 & 0.05 & 15.68 & 0.03 & 0.40 & 0.06 & 16.08 & 0.05 & 15.68 & 0.03 \\
\hline 248 & NGC4096 & 0.02 & 492 & 119 & 20 & 14.08 & 0.05 & 13.59 & 0.03 & 0.49 & 0.06 & 14.10 & 0.05 & 13.61 & 0.03 \\
\hline 249 & KUG1207+367 & 0.03 & 54 & 20 & -10 & 17.16 & 0.05 & 16.71 & 0.03 & 0.45 & 0.06 & 17.15 & 0.05 & 16.67 & 0.03 \\
\hline
\end{tabular}


Table 2

(Continued)

\begin{tabular}{|c|c|c|c|c|c|c|c|c|c|c|c|c|c|c|c|}
\hline \multirow{2}{*}{$\begin{array}{l}\text { No. } \\
\text { (1) }\end{array}$} & \multirow{2}{*}{$\begin{array}{c}\text { Galaxy Name } \\
\text { (2) }\end{array}$} & \multirow{2}{*}{$\begin{array}{c}E(B-V) \\
\text { (3) }\end{array}$} & \multirow{2}{*}{$\begin{array}{l}a \\
(") \\
(4) \\
\end{array}$} & \multirow{2}{*}{$\begin{array}{l}b \\
\left({ }^{\prime \prime}\right) \\
(5)\end{array}$} & \multirow{2}{*}{$\begin{array}{l}\text { P.A. } \\
\text { (deg) } \\
(6)\end{array}$} & \multicolumn{2}{|c|}{$\begin{array}{l}\text { FUV }_{\text {ap }} \\
(\mathrm{mag})\end{array}$} & \multicolumn{2}{|c|}{$\begin{array}{c}\mathrm{NUV}_{\text {ap }} \\
(\mathrm{mag})\end{array}$} & \multicolumn{2}{|c|}{$\begin{array}{c}(\mathrm{FUV}- \\
\mathrm{NUV})_{\mathrm{ap}} \\
(\mathrm{mag})\end{array}$} & $\begin{array}{l}\text { FUV } \\
(\mathrm{m}\end{array}$ & $\begin{array}{l}\text { sym } \\
\text { g) }\end{array}$ & (II) & \\
\hline & & & & & & (7) & (8) & (9) & $(10)$ & (11) & $(12)$ & (13) & (14) & $(15)$ & $(16)$ \\
\hline 250 & NGC4144 & 0.01 & 444 & 96 & -76 & 13.95 & 0.05 & 13.56 & 0.04 & 0.39 & 0.06 & 13.96 & 0.05 & 13.58 & 0.04 \\
\hline 251 & NGC4163 & 0.02 & 114 & 101 & 10 & 15.34 & 0.05 & 14.95 & 0.03 & 0.39 & 0.06 & 15.34 & 0.05 & 14.95 & 0.03 \\
\hline 252 & NGC4190 & 0.03 & 120 & 92 & 30 & 14.77 & 0.05 & 14.32 & 0.03 & 0.45 & 0.06 & 14.77 & 0.05 & 14.32 & 0.03 \\
\hline 253 & UGC7242 & 0.02 & 132 & 53 & -8 & 16.05 & 0.05 & 15.51 & 0.06 & 0.54 & 0.08 & 16.05 & 0.05 & 15.52 & 0.06 \\
\hline 254 & UGCA276 & 0.02 & 66 & 66 & 0 & $(23.55)$ & & 18.62 & 0.06 & $\ldots$ & . & . & 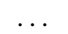 & 18.47 & 0.16 \\
\hline 255 & NGC4204 & 0.03 & 264 & 213 & -60 & 14.31 & 0.08 & 14.06 & 0.03 & 0.25 & 0.08 & 14.33 & 0.08 & 14.07 & 0.03 \\
\hline 256 & UGC7267 & 0.02 & 90 & 34 & 53 & 16.30 & 0.05 & 15.96 & 0.03 & 0.34 & 0.06 & 16.29 & 0.05 & 15.94 & 0.03 \\
\hline 257 & UGC7271 & 0.01 & 144 & 43 & -20 & 16.46 & 0.05 & 16.14 & 0.04 & 0.32 & 0.06 & 16.47 & 0.05 & 16.16 & 0.04 \\
\hline 258 & NGC4214 & 0.02 & 504 & 391 & -40 & 11.47 & 0.05 & 11.27 & 0.03 & 0.20 & 0.06 & 11.51 & 0.05 & 11.29 & 0.04 \\
\hline 259 & CGCG269-049 & 0.02 & 72 & 42 & -54 & 16.60 & 0.05 & 16.35 & 0.03 & 0.25 & 0.06 & 16.59 & 0.05 & 16.34 & 0.03 \\
\hline 260 & UGC7298 & 0.02 & 90 & 42 & -40 & 17.26 & 0.05 & 16.95 & 0.06 & 0.31 & 0.08 & 17.26 & 0.05 & 16.93 & 0.06 \\
\hline 261 & NGC4236 & 0.01 & 936 & 308 & -18 & 11.76 & 0.05 & 11.57 & 0.03 & 0.19 & 0.06 & 11.79 & 0.06 & 11.60 & 0.04 \\
\hline 262 & NGC4244 & 0.02 & 990 & 126 & 48 & 12.67 & 0.05 & 12.20 & 0.03 & 0.47 & 0.06 & 12.68 & 0.05 & 12.20 & 0.03 \\
\hline 263 & NGC4242 & 0.01 & 222 & 169 & 25 & 13.88 & 0.05 & 13.53 & 0.03 & 0.35 & 0.06 & 13.89 & 0.05 & 13.54 & 0.03 \\
\hline 264 & NGC4248 & 0.02 & 144 & 53 & -72 & 16.84 & 0.05 & 15.87 & 0.04 & 0.97 & 0.06 & 16.84 & 0.05 & 15.83 & 0.04 \\
\hline 265 & IC3104 & 0.41 & $\ldots$ & $\ldots$ & $\ldots$ & $\ldots$ & $\ldots$ & $\ldots$ & $\ldots$ & $\ldots$ & $\ldots$ & $\ldots$ & $\ldots$ & $\ldots$ & $\ldots$ \\
\hline 266 & NGC4258 & 0.02 & 834 & 323 & -30 & 11.82 & 0.05 & 11.40 & 0.03 & 0.42 & 0.06 & 11.82 & 0.05 & 11.39 & 0.03 \\
\hline 267 & UGC7356 & 0.02 & 48 & 48 & 0 & $\ldots$ & $\ldots$ & 18.69 & 0.05 & $\ldots$ & $\ldots$ & $\ldots$ & $\ldots$ & 18.59 & 0.06 \\
\hline 268 & ISZ399 & 0.06 & $\ldots$ & $\ldots$ & $\ldots$ & $\ldots$ & $\ldots$ & $\ldots$ & $\ldots$ & $\ldots$ & $\ldots$ & $\ldots$ & $\ldots$ & $\ldots$ & .. \\
\hline 269 & NGC4288 & 0.01 & 186 & 133 & -50 & 14.57 & 0.05 & 14.35 & 0.03 & 0.22 & 0.06 & 14.57 & 0.05 & 14.37 & 0.03 \\
\hline 270 & UGC7408 & 0.01 & 180 & 83 & -80 & 16.10 & 0.05 & 15.48 & 0.04 & 0.62 & 0.06 & 16.10 & 0.05 & 15.47 & 0.04 \\
\hline 271 & UGC7490 & 0.02 & $\ldots$ & $\ldots$ & $\ldots$ & $\ldots$ & $\ldots$ & $\ldots$ & $\ldots$ & $\ldots$ & $\ldots$ & $\ldots$ & $\ldots$ & $\ldots$ & $\ldots$ \\
\hline 272 & LEDA166137 & 0.02 & 60 & 20 & 80 & 17.24 & 0.08 & 16.97 & 0.05 & 0.27 & 0.09 & 17.08 & 0.20 & 16.84 & 0.05 \\
\hline 273 & NGC4395 & 0.02 & 588 & 490 & -33 & 11.63 & 0.05 & 11.51 & 0.03 & 0.12 & 0.06 & 11.62 & 0.05 & 11.50 & 0.03 \\
\hline 274 & UGCA281 & 0.01 & 66 & 50 & -85 & 15.17 & 0.05 & 15.16 & 0.03 & 0.01 & 0.06 & 15.17 & 0.05 & 15.16 & 0.03 \\
\hline 275 & UGC7559 & 0.01 & 222 & 139 & -20 & 15.09 & 0.06 & 15.00 & 0.03 & 0.09 & 0.07 & 15.06 & 0.06 & 14.98 & 0.03 \\
\hline 276 & UGC7577 & 0.02 & 192 & 107 & -50 & 14.82 & 0.05 & 14.49 & 0.03 & 0.33 & 0.06 & 14.82 & 0.05 & 14.48 & 0.03 \\
\hline 277 & UGC7584 & 0.02 & 72 & 40 & -60 & 17.01 & 0.05 & 16.70 & 0.03 & 0.31 & 0.06 & 17.01 & 0.05 & 16.68 & 0.03 \\
\hline 278 & LSBCF573-01 & 0.02 & 42 & 30 & 80 & 18.12 & 0.06 & 17.75 & 0.03 & 0.37 & 0.07 & 18.10 & 0.07 & 17.66 & 0.03 \\
\hline 279 & NGC4449 & 0.02 & 462 & 328 & 45 & 10.83 & 0.05 & 10.80 & 0.03 & 0.03 & 0.06 & 10.84 & 0.05 & 10.82 & 0.03 \\
\hline 280 & UGC7599 & 0.02 & 144 & 72 & -45 & 16.04 & 0.05 & 15.96 & 0.04 & 0.08 & 0.07 & 16.05 & 0.05 & 15.96 & 0.04 \\
\hline 281 & UGC7605 & 0.01 & 108 & 78 & 30 & 15.78 & 0.05 & 15.62 & 0.03 & 0.16 & 0.06 & 15.78 & 0.05 & 15.62 & 0.03 \\
\hline 282 & NGC4455 & 0.02 & 204 & 58 & 16 & 14.43 & 0.05 & 14.16 & 0.03 & 0.27 & 0.06 & 14.43 & 0.05 & 14.17 & 0.03 \\
\hline 283 & UGC7608 & 0.02 & 150 & 146 & 60 & 14.74 & 0.06 & 14.70 & 0.03 & 0.04 & 0.07 & 14.74 & 0.06 & 14.68 & 0.03 \\
\hline 284 & NGC4460 & 0.02 & 174 & 52 & 40 & 15.53 & 0.05 & 14.77 & 0.03 & 0.76 & 0.06 & 15.53 & 0.05 & 14.76 & 0.03 \\
\hline 285 & MCG+07-26-011 & 0.02 & 48 & 27 & -30 & 17.26 & 0.05 & 16.77 & 0.03 & 0.49 & 0.06 & 17.26 & 0.05 & 16.74 & 0.03 \\
\hline 286 & UGC7639 & 0.01 & 102 & 71 & -27 & 16.13 & 0.05 & 15.72 & 0.03 & 0.41 & 0.06 & 16.15 & 0.05 & 15.69 & 0.03 \\
\hline 287 & $1 \mathrm{CG}+07-26-012$ & 0.02 & 66 & 22 & -68 & 17.16 & 0.05 & 16.76 & 0.03 & 0.40 & 0.06 & 17.15 & 0.05 & 16.73 & 0.04 \\
\hline 288 & NGC4485 & 0.02 & 204 & 142 & 15 & 13.47 & 0.05 & 13.28 & 0.03 & 0.19 & 0.06 & 13.51 & 0.05 & 13.34 & 0.03 \\
\hline 289 & NGC4490 & 0.02 & 468 & 230 & -55 & 11.98 & 0.05 & 11.53 & 0.03 & 0.45 & 0.06 & 12.02 & 0.05 & 11.55 & 0.03 \\
\hline 290 & UGC7678 & 0.02 & 66 & 47 & 80 & 15.42 & 0.06 & 15.12 & 0.03 & 0.30 & 0.07 & 15.39 & 0.06 & 15.11 & 0.03 \\
\hline 291 & UGC7690 & 0.03 & 126 & 96 & 20 & 14.66 & 0.05 & 14.29 & 0.03 & 0.37 & 0.06 & 14.67 & 0.05 & 14.30 & 0.03 \\
\hline 292 & UGC7699 & 0.01 & 282 & 74 & 32 & 14.72 & 0.05 & 14.43 & 0.04 & 0.29 & 0.06 & 14.73 & 0.05 & 14.45 & 0.04 \\
\hline 293 & UGC7698 & 0.02 & 276 & 190 & -10 & 14.70 & 0.06 & 14.54 & 0.03 & 0.16 & 0.07 & 14.71 & 0.06 & 14.55 & 0.03 \\
\hline 294 & UGC7719 & 0.02 & 138 & 87 & -17 & 15.93 & 0.05 & 15.73 & 0.04 & 0.20 & 0.06 & 15.95 & 0.05 & 15.75 & 0.04 \\
\hline 295 & NGC4534 & 0.01 & 192 & 155 & -55 & 13.92 & 0.05 & 13.82 & 0.03 & 0.10 & 0.06 & 13.91 & 0.05 & 13.81 & 0.03 \\
\hline 296 & UGC7774 & 0.02 & 156 & 22 & -78 & 16.33 & 0.05 & 15.76 & 0.03 & 0.57 & 0.06 & 16.32 & 0.05 & 15.74 & 0.03 \\
\hline 297 & UGCA290 & 0.01 & 48 & 24 & 42 & 15.88 & 0.06 & 15.92 & 0.04 & -0.04 & 0.07 & 15.84 & 0.09 & 15.89 & 0.05 \\
\hline 298 & UGCA292 & 0.02 & 114 & 80 & 35 & 16.20 & 0.05 & 16.21 & 0.03 & -0.01 & 0.06 & 16.21 & 0.05 & 16.21 & 0.03 \\
\hline 299 & M104 & 0.05 & 390 & 157 & 90 & 14.54 & 0.06 & 13.39 & 0.05 & 1.15 & 0.08 & 14.56 & 0.07 & 13.37 & 0.05 \\
\hline 300 & NGC4605 & 0.01 & 588 & 223 & -55 & 12.97 & 0.06 & 12.56 & 0.04 & 0.41 & 0.08 & 13.01 & 0.06 & 12.59 & 0.04 \\
\hline 301 & NGC4618 & 0.02 & 312 & 253 & 25 & 12.80 & 0.05 & 12.54 & 0.03 & 0.26 & 0.06 & 12.83 & 0.05 & 12.56 & 0.03 \\
\hline 302 & NGC4625 & 0.02 & 240 & 207 & -30 & 14.49 & 0.05 & 14.20 & 0.04 & 0.29 & 0.06 & 14.41 & 0.07 & 14.15 & 0.05 \\
\hline 303 & NGC4631 & 0.02 & 1158 & 202 & 86 & 11.29 & 0.05 & 11.07 & 0.03 & 0.22 & 0.06 & 11.31 & 0.05 & 11.09 & 0.03 \\
\hline 304 & UGC7866 & 0.02 & 150 & 132 & 0 & 14.62 & 0.05 & 14.54 & 0.03 & 0.08 & 0.06 & 14.62 & 0.05 & 14.54 & 0.03 \\
\hline 305 & NGC4656 & 0.01 & 1128 & 224 & 33 & 11.47 & 0.05 & 11.48 & 0.03 & -0.01 & 0.06 & 11.48 & 0.05 & 11.48 & 0.03 \\
\hline 306 & UGC7916 & 0.02 & 186 & 126 & 0 & 15.73 & 0.05 & 15.56 & 0.06 & 0.17 & 0.08 & 15.75 & 0.05 & 15.54 & 0.06 \\
\hline 307 & ESO381-G020 & 0.07 & 126 & 50 & -42 & 15.12 & 0.09 & 14.84 & 0.04 & 0.28 & 0.10 & 15.06 & 0.12 & 14.82 & 0.04 \\
\hline 308 & UGCA298 & 0.01 & 36 & 22 & 45 & 17.70 & 0.09 & 17.00 & 0.04 & 0.70 & 0.10 & 17.73 & 0.11 & 17.00 & 0.06 \\
\hline 309 & UGC7950 & 0.02 & 78 & 60 & 5 & 15.23 & 0.05 & 15.01 & 0.03 & 0.22 & 0.06 & 15.23 & 0.05 & 15.02 & 0.03 \\
\hline 310 & UGC7949 & 0.02 & 144 & 115 & 60 & 15.95 & 0.06 & 15.81 & 0.03 & 0.14 & 0.07 & 15.94 & 0.06 & 15.80 & 0.03 \\
\hline 311 & NGC4707 & 0.01 & 162 & 155 & 25 & 15.05 & 0.05 & 14.92 & 0.04 & 0.13 & 0.06 & 15.06 & 0.05 & 14.93 & 0.04 \\
\hline
\end{tabular}


Table 2

(Continued)

\begin{tabular}{|c|c|c|c|c|c|c|c|c|c|c|c|c|c|c|c|}
\hline \multirow{2}{*}{$\begin{array}{l}\text { No. } \\
\text { (1) }\end{array}$} & \multirow{2}{*}{$\begin{array}{c}\text { Galaxy Name } \\
\text { (2) }\end{array}$} & \multirow{2}{*}{$\begin{array}{c}E(B-V) \\
(3)\end{array}$} & \multirow{2}{*}{$\begin{array}{l}a \\
(") \\
(4)\end{array}$} & \multirow{2}{*}{$\begin{array}{l}b \\
(\prime) \\
(5) \\
\end{array}$} & \multirow{2}{*}{$\begin{array}{l}\text { P.A. } \\
(\mathrm{deg}) \\
(6)\end{array}$} & \multicolumn{2}{|c|}{$\begin{array}{l}\mathrm{FUV}_{\text {ap }} \\
(\mathrm{mag})\end{array}$} & \multicolumn{2}{|c|}{$\begin{array}{l}\mathrm{NUV}_{\text {ap }} \\
(\mathrm{mag})\end{array}$} & \multicolumn{2}{|c|}{$\begin{array}{c}(\mathrm{FUV}- \\
\mathrm{NUV})_{\mathrm{ap}} \\
(\mathrm{mag})\end{array}$} & $\begin{array}{r}\text { FUV } \\
(\mathrm{m}\end{array}$ & $\begin{array}{l}\text { asym } \\
\text { g) }\end{array}$ & $\mathrm{NU}$ & \\
\hline & & & & & & (7) & (8) & (9) & $(10)$ & $(11)$ & $(12)$ & (13) & $(14)$ & (15) & $(16)$ \\
\hline 312 & NGC4736 & 0.02 & 834 & 677 & -75 & 11.75 & 0.05 & 11.35 & 0.04 & 0.40 & 0.06 & 11.74 & 0.05 & 11.42 & 0.05 \\
\hline 313 & UGC8024 & 0.01 & 204 & 150 & 35 & 14.79 & 0.05 & 14.82 & 0.06 & -0.03 & 0.08 & 14.80 & 0.05 & 14.82 & 0.06 \\
\hline 314 & UGC8055 & 0.03 & 66 & 51 & 40 & 16.70 & 0.07 & 16.57 & 0.05 & 0.13 & 0.09 & 16.61 & 0.16 & 16.48 & 0.14 \\
\hline 315 & NGC4826 & 0.04 & 396 & 214 & -65 & 13.51 & 0.08 & 12.55 & 0.03 & 0.96 & 0.08 & 13.57 & 0.08 & 12.55 & 0.03 \\
\hline 316 & UGC8091 & 0.03 & 72 & 65 & 30 & 15.21 & 0.05 & 15.16 & 0.04 & 0.05 & 0.06 & 15.20 & 0.05 & 15.15 & 0.04 \\
\hline 317 & UGC8146 & 0.01 & 258 & 29 & 30 & 15.71 & 0.05 & 15.34 & 0.03 & 0.37 & 0.06 & 15.72 & 0.05 & 15.36 & 0.03 \\
\hline 318 & UGCA319 & 0.08 & 60 & 41 & 35 & 16.58 & 0.05 & 16.10 & 0.03 & 0.48 & 0.06 & 16.57 & 0.05 & 16.03 & 0.04 \\
\hline 319 & UGCA320 & 0.08 & 414 & 52 & -60 & 13.93 & 0.09 & 13.71 & 0.04 & 0.22 & 0.09 & 13.91 & 0.09 & 13.69 & 0.04 \\
\hline 320 & NGC4945 & 0.18 & $\ldots$ & $\ldots$ & $\ldots$ & $\ldots$ & $\ldots$ & $\ldots$ & $\ldots$ & $\ldots$ & $\ldots$ & $\ldots$ & $\ldots$ & $\ldots$ & $\ldots$ \\
\hline 321 & UGC8188 & 0.01 & 264 & 242 & -50 & 13.35 & 0.05 & 13.14 & 0.04 & 0.21 & 0.06 & 13.35 & 0.05 & 13.16 & 0.04 \\
\hline 322 & UGC8201 & 0.02 & 246 & 134 & 90 & 14.49 & 0.05 & 14.16 & 0.03 & 0.33 & 0.06 & 14.51 & 0.05 & 14.16 & 0.03 \\
\hline 323 & ICG-03-34-002 & 0.08 & 60 & 34 & -40 & 16.10 & 0.05 & 15.75 & 0.03 & 0.35 & 0.06 & 16.09 & 0.05 & 15.69 & 0.04 \\
\hline 324 & UGC8215 & 0.01 & 48 & 34 & 60 & 17.64 & 0.05 & 17.34 & 0.03 & 0.30 & 0.06 & 17.62 & 0.05 & 17.29 & 0.03 \\
\hline 325 & UGC8245 & 0.03 & 90 & 37 & 75 & 16.49 & 0.05 & 16.01 & 0.03 & 0.48 & 0.06 & 16.48 & 0.05 & 15.97 & 0.03 \\
\hline 326 & NGC5023 & 0.02 & 444 & 44 & 28 & 14.65 & 0.05 & 14.25 & 0.03 & 0.40 & 0.06 & 14.66 & 0.05 & 14.25 & 0.03 \\
\hline 327 & CGCG217-018 & 0.01 & 48 & 34 & 50 & 16.60 & 0.05 & 16.15 & 0.03 & 0.45 & 0.06 & 16.59 & 0.05 & 16.11 & 0.03 \\
\hline 328 & UGC8308 & 0.01 & 114 & 62 & -25 & 16.31 & 0.05 & 16.24 & 0.03 & 0.07 & 0.06 & 16.31 & 0.05 & 16.24 & 0.03 \\
\hline 329 & UGC8313 & 0.01 & 120 & 35 & 35 & 16.32 & 0.05 & 16.00 & 0.03 & 0.32 & 0.06 & 16.32 & 0.05 & 15.99 & 0.03 \\
\hline 330 & UGC8320 & 0.02 & 264 & 103 & -30 & 14.53 & 0.05 & 14.18 & 0.05 & 0.35 & 0.07 & 14.52 & 0.05 & 14.20 & 0.05 \\
\hline 331 & UGC8331 & 0.01 & 318 & 106 & -40 & 15.70 & 0.05 & 15.47 & 0.10 & 0.23 & 0.12 & 15.71 & 0.06 & 15.56 & 0.16 \\
\hline 332 & NGC5055 & 0.02 & 942 & 538 & -75 & 12.30 & 0.05 & 11.74 & 0.04 & 0.56 & 0.06 & 12.32 & 0.05 & 11.82 & 0.04 \\
\hline 333 & NGC5068 & 0.10 & 306 & 268 & -70 & 11.94 & 0.09 & 11.55 & 0.04 & 0.39 & 0.10 & 11.93 & 0.09 & 11.53 & 0.04 \\
\hline 334 & NGC5102 & 0.05 & $\ldots$ & $\ldots$ & $\ldots$ & $\ldots$ & $\ldots$ & $\ldots$ & $\ldots$ & $\ldots$ & $\ldots$ & $\ldots$ & $\ldots$ & $\ldots$ & $\ldots$ \\
\hline 335 & NGC5128 & 0.11 & 396 & 308 & 35 & 11.95 & 0.05 & 11.04 & 0.03 & 0.91 & 0.06 & 11.89 & 0.05 & 10.87 & 0.03 \\
\hline 336 & IC4247 & 0.06 & 90 & 35 & -22 & 15.96 & 0.05 & 15.54 & 0.03 & 0.42 & 0.06 & 15.96 & 0.05 & 15.52 & 0.03 \\
\hline 337 & ESO324-G024 & 0.11 & 138 & 99 & 50 & 14.45 & 0.06 & 14.00 & 0.06 & 0.45 & 0.09 & 14.41 & 0.09 & 13.93 & 0.06 \\
\hline 338 & NGC5204 & 0.01 & 222 & 133 & 5 & 12.92 & 0.05 & 12.70 & 0.03 & 0.22 & 0.06 & 12.92 & 0.05 & 12.68 & 0.03 \\
\hline 339 & NGC5194 & 0.04 & 498 & 307 & -17 & 11.03 & 0.05 & 10.55 & 0. & 0.48 & 0.06 & 11.05 & 0.05 & 10.57 & 0.03 \\
\hline 340 & NGC & 0.04 & 258 & 205 & 79 & 14.64 & 0.06 & 13.81 & 0.03 & 0.83 & 0.07 & 14.10 & 0.16 & 13.46 & 0.09 \\
\hline 341 & UGC8508 & 0.02 & $\ldots$ & $\ldots$ & $\ldots$ & $\ldots$ & $\ldots$ & $\ldots$ & $\ldots$ & $\ldots$ & $\ldots$ & $\ldots$ & $\ldots$ & $\ldots$ & $\ldots$ \\
\hline 342 & SBS1331+493 & 0.01 & $\ldots$ & $\ldots$ & $\ldots$ & $\ldots$ & $\ldots$ & ... & $\ldots$ & $\ldots$ & $\ldots$ & $\ldots$ & $\ldots$ & $\cdots$ & $\cdots$ \\
\hline 343 & NGC5206 & 0.12 & $\ldots$ & $\ldots$ & $\ldots$ & $\ldots$ & $\ldots$ & $\ldots$ & $\ldots$ & $\ldots$ & $\ldots$ & $\ldots$ & $\ldots$ & $\ldots$ & $\ldots$ \\
\hline 344 & NGC5229 & 0.02 & 246 & 30 & -13 & 15.71 & 0.06 & 15.36 & 0.03 & 0.35 & 0.07 & 15.73 & 0.06 & 15.36 & 0.03 \\
\hline 345 & NGC5238 & 0.01 & 126 & 74 & -20 & 15.19 & 0.05 & 14.97 & 0.03 & 0.22 & 0.06 & 15.20 & 0.05 & 14.98 & 0.03 \\
\hline 346 & ESO270-G017 & 0.11 & $\ldots$ & $\ldots$ & $\ldots$ & $\ldots$ & $\ldots$ & $\ldots$ & $\ldots$ & $\ldots$ & $\ldots$ & $\ldots$ & $\ldots$ & $\ldots$ & $\ldots$ \\
\hline 347 & [KK98]208 & 0.04 & & . & 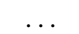 & $(23.75)$ & & $(23.90)$ & $\ldots$ & $\ldots$ & $\ldots$ & $\ldots$ & $\ldots$ & $\ldots$ & $\ldots$ \\
\hline 348 & NGC5236 & 0.07 & 1158 & 1032 & 0 & 10.07 & 0.05 & 9.51 & 0.04 & 0.56 & 0.06 & 10.11 & 0.05 & 9.57 & 0.04 \\
\hline 349 & ESO444-G084 & 0.07 & 72 & 55 & -75 & 16.02 & 0.06 & 15.77 & 0.03 & 0.25 & 0.07 & 15.98 & 0.07 & 15.73 & 0.03 \\
\hline 350 & UGC8638 & 0.01 & 84 & 56 & 70 & 15.76 & 0.05 & 15.58 & 0.03 & 0.18 & 0.06 & 15.77 & 0.05 & 15.58 & 0.03 \\
\hline 351 & UGC8651 & 0.01 & 168 & 95 & 80 & 15.57 & 0.05 & 15.45 & 0. & 0.12 & 0.06 & 15.59 & 0.05 & 15.45 & 0.03 \\
\hline 352 & NGC5253 & 0.06 & 372 & 141 & 45 & 12.32 & 0.05 & 11.91 & 0.03 & 0.41 & 0.06 & 12.36 & 0.05 & 11.94 & 0.03 \\
\hline 353 & IC4316 & 0.05 & 60 & 39 & 50 & 16.10 & 0.06 & 15.96 & 0.04 & 0.14 & 0.07 & 16.10 & 0.06 & 15.93 & 0.04 \\
\hline 354 & NGC5264 & 0.05 & 186 & 112 & 65 & 14.90 & 0.05 & 14.28 & 0.08 & 0.62 & 0.09 & 14.92 & 0.05 & 14.35 & 0.08 \\
\hline 355 & UGC8683 & 0.01 & 156 & 104 & -43 & 16.04 & 0.06 & 15.81 & 0.03 & 0.23 & 0.07 & 15.99 & 0.08 & 15.74 & 0.04 \\
\hline 356 & ESO325-G011 & 0.09 & $\ldots$ & $\ldots$ & $\ldots$ & $\ldots$ & $\ldots$ & $\ldots$ & $\ldots$ & $\ldots$ & $\ldots$ & $\ldots$ & $\ldots$ & $\ldots$ & $\cdots$ \\
\hline 357 & ESO383-G087 & 0.07 & $\ldots$ & $\ldots$ & $\ldots$ & $\ldots$ & $\ldots$ & $\ldots$ & $\ldots$ & $\ldots$ & $\ldots$ & $\ldots$ & $\ldots$ & $\ldots$ & $\ldots$ \\
\hline 358 & ESO383-G091 & 0.08 & 100 & 100 & -78 & 17.25 & 0.12 & $\ldots$ & $\ldots$ & $\ldots$ & $\ldots$ & 17.18 & 0.23 & 16.39 & 0.04 \\
\hline 359 & UGC8760 & 0.02 & 162 & 52 & 33 & 15.83 & 0.05 & 15.60 & 0.04 & 0.23 & 0.06 & 15.84 & 0.05 & 15.60 & 0.04 \\
\hline 360 & UGC8837 & .01 & $\ldots$ & $\ldots$ & $\ldots$ & $\ldots$ & $\ldots$ & $\ldots$ & $\ldots$ & $\ldots$ & $\ldots$ & $\ldots$ & $\ldots$ & $\ldots$ & $\ldots$ \\
\hline 361 & UGC8833 & 0.01 & 60 & 53 & -40 & 16.64 & 0.05 & 16.42 & 0.04 & 0.22 & 0.06 & 16.63 & 0.05 & 16.41 & 0.04 \\
\hline 362 & ESO384-G016 & 0.07 & 24 & 17 & 67 & 18.53 & 0.23 & 17.50 & 0.08 & 1.03 & 0.24 & 18.60 & 0.24 & 17.31 & 0.10 \\
\hline 363 & NGC5457 & 0.01 & 1290 & 1205 & 90 & 9.96 & 0.05 & 9.80 & 0.03 & 0.16 & 0.06 & 9.96 & 0.05 & 9.81 & 0.03 \\
\hline 364 & NGC5408 & 0.07 & $\ldots$ & $\ldots$ & $\ldots$ & $\ldots$ & $\ldots$ & $\ldots$ & $\ldots$ & $\ldots$ & $\ldots$ & $\ldots$ & $\ldots$ & $\ldots$ & $\ldots$ \\
\hline 365 & NGC5474 & 0.01 & 342 & 306 & 0 & 12.89 & 0.05 & 12.79 & 0.04 & 0.10 & 0.07 & 12.90 & 0.05 & 12.81 & 0.04 \\
\hline 366 & NGC5477 & 0.01 & 102 & 78 & -85 & 14.87 & 0.06 & 14.88 & 0.04 & -0.01 & 0.07 & 14.87 & 0.06 & 14.88 & 0.04 \\
\hline 367 & KKR03 & 0.01 & 36 & 30 & -20 & 18.38 & 0.06 & 18.17 & 0.04 & 0.21 & 0.07 & 18.12 & 0.08 & 18.04 & 0.04 \\
\hline 368 & Circinus & 1.46 & .. & $\ldots$ & $\ldots$ & & & . & & $\ldots$ & & & & & \\
\hline 369 & KUG1413+573 & 0.01 & 48 & 18 & 0 & 17.27 & 0.08 & 17.10 & 0.05 & 0.17 & 0.09 & 17.28 & 0.11 & 17.07 & 0.06 \\
\hline 370 & UGC9128 & 0.02 & 120 & 92 & 45 & 16.21 & 0.05 & 15.83 & 0.03 & 0.38 & 0.06 & 16.21 & 0.05 & 15.83 & 0.03 \\
\hline 371 & SBS1415+437 & 0.01 & 72 & 18 & 25 & 15.93 & 0.06 & 15.77 & 0.04 & 0.16 & 0.07 & 15.92 & 0.06 & 15.75 & 0.04 \\
\hline 372 & NGC5585 & 0.02 & 432 & 276 & 30 & 13.03 & 0.05 & 12.72 & 0.03 & 0.31 & 0.06 & 13.03 & 0.05 & 12.72 & 0.03 \\
\hline 373 & UGC9211 & 0.01 & 72 & 59 & -65 & 16.08 & 0.06 & 15.89 & 0.04 & 0.19 & 0.08 & 15.90 & 0.16 & 15.76 & 0.05 \\
\hline
\end{tabular}


Table 2

(Continued)

\begin{tabular}{|c|c|c|c|c|c|c|c|c|c|c|c|c|c|c|c|}
\hline \multirow{2}{*}{$\begin{array}{l}\text { No. } \\
\text { (1) } \\
\end{array}$} & \multirow{2}{*}{$\begin{array}{c}\text { Galaxy Name } \\
\text { (2) }\end{array}$} & \multirow{2}{*}{$\begin{array}{c}E(B-V) \\
(3)\end{array}$} & \multirow{2}{*}{$\begin{array}{l}a \\
(") \\
(4) \\
\end{array}$} & \multirow{2}{*}{$\begin{array}{l}b \\
(1 ") \\
(5) \\
\end{array}$} & \multirow{2}{*}{$\begin{array}{l}\text { P.A. } \\
\text { (deg) } \\
(6)\end{array}$} & \multicolumn{2}{|c|}{$\begin{array}{l}\mathrm{FUV}_{\text {ap }} \\
(\mathrm{mag})\end{array}$} & \multicolumn{2}{|c|}{$\begin{array}{c}\mathrm{NUV}_{\text {ap }} \\
(\mathrm{mag})\end{array}$} & \multicolumn{2}{|c|}{$\begin{array}{l}\text { (FUV - } \\
\text { NUV) })_{\text {ap }} \\
(\mathrm{mag})\end{array}$} & \multicolumn{2}{|c|}{$\begin{array}{c}\text { FUV }_{\text {asym }} \\
(\mathrm{mag})\end{array}$} & $\begin{array}{r}\mathrm{NU} \\
(\mathrm{m}\end{array}$ & \\
\hline & & & & & & (7) & $(8)$ & (9) & (10) & (11) & (12) & (13) & (14) & $(15)$ & (16) \\
\hline 374 & NGC5608 & 0.01 & 108 & 54 & -85 & 15.12 & 0.05 & 14.88 & 0.04 & 0.24 & 0.06 & 15.11 & 0.05 & 14.87 & 0.04 \\
\hline 375 & UGC9240 & 0.01 & 132 & 132 & 0 & 14.80 & 0.05 & 14.55 & 0.03 & 0.25 & 0.06 & 14.82 & 0.05 & 14.55 & 0.03 \\
\hline 376 & UKS1424-460 & 0.13 & $\ldots$ & $\ldots$ & .. & $\ldots$ & $\ldots$ & $\ldots$ & $\ldots$ & $\ldots$ & $\ldots$ & $\ldots$ & $\ldots$ & $\ldots$ & $\ldots$ \\
\hline 377 & ESO222-G010 & 0.27 & $\ldots$ & $\ldots$ & $\ldots$ & $\ldots$ & $\ldots$ & $\ldots$ & $\ldots$ & $\ldots$ & $\ldots$ & 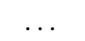 & $\ldots$ & $\ldots$ & \\
\hline 378 & UGC9405 & 0.01 & 126 & 44 & -35 & 16.74 & 0.06 & 16.37 & 0.03 & 0.37 & 0.07 & 16.71 & 0.07 & 16.30 & 0.07 \\
\hline 379 & MRK475 & 0.01 & 30 & 22 & 80 & 17.39 & 0.05 & 17.16 & 0.03 & 0.23 & 0.06 & 17.38 & 0.05 & 17.14 & 0.03 \\
\hline 380 & ESO272-G025 & 0.16 & $\ldots$ & $\ldots$ & $\ldots$ & $\ldots$ & $\ldots$ & $\ldots$ & $\ldots$ & $\ldots$ & $\ldots$ & $\ldots$ & $\ldots$ & $\ldots$ & \\
\hline 381 & UGC9497 & 0.01 & 72 & 14 & 63 & 16.33 & 0.06 & 16.21 & 0.04 & 0.12 & 0.07 & 16.32 & 0.06 & 16.20 & 0.04 \\
\hline 382 & NGC5832 & 0.03 & 162 & 96 & 45 & 14.56 & 0.05 & 14.28 & 0.03 & 0.28 & 0.06 & 14.55 & 0.05 & 14.26 & 0.03 \\
\hline 383 & ESO223-G009 & 0.26 & $\ldots$ & $\ldots$ & $\ldots$ & $\ldots$ & & $\ldots$ & $\ldots$ & $\ldots$ & $\ldots$ & & $\ldots$ & & \\
\hline 384 & UGC9660 & 0.02 & 72 & 36 & 83 & 15.82 & 0.05 & 15.48 & 0.03 & 0.34 & 0.06 & 15.82 & 0.05 & 15.47 & 0.03 \\
\hline 385 & ESO274-G001 & 0.25 & $\ldots$ & $\ldots$ & $\ldots$ & $\ldots$ & . & $\ldots$ & $\ldots$ & $\ldots$ & $\ldots$ & $\ldots$ & $\ldots$ & $\ldots$ & $\ldots$ \\
\hline 386 & NGC5949 & 0.02 & 162 & 74 & -33 & 15.14 & 0.05 & 14.70 & 0.03 & 0.44 & 0.06 & 15.15 & 0.05 & 14.73 & 0.03 \\
\hline 387 & UGC9893 & 0.01 & 78 & 28 & 42 & 16.66 & 0.05 & 16.29 & 0.03 & 0.37 & 0.06 & 16.66 & 0.05 & 16.27 & 0.03 \\
\hline 388 & UGC9992 & 0.04 & 114 & 71 & -20 & 16.30 & 0.07 & 16.00 & 0.03 & 0.30 & 0.08 & 16.31 & 0.09 & 15.98 & 0.03 \\
\hline 389 & LEDA100404 & 0.05 & $\ldots$ & $\ldots$ & $\ldots$ & $\ldots$ & $\ldots$ & $\ldots$ & $\ldots$ & $\ldots$ & $\ldots$ & $\ldots$ & $\ldots$ & $\ldots$ & $\ldots$ \\
\hline 390 & ESO137-G018 & 0.25 & $\ldots$ & $\cdots$ & $\ldots$ & $\ldots$ & $\ldots$ & $\ldots$ & $\ldots$ & $\ldots$ & $\ldots$ & $\ldots$ & $\ldots$ & $\ldots$ & $\ldots$ \\
\hline 391 & ESO179-IG013 & 0.27 & $\ldots$ & .. & .. & $\ldots$ & $\ldots$ & .. & $\ldots$ & $\ldots$ & $\ldots$ & .. & $\ldots$ & 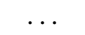 & \\
\hline 392 & UGC10669 & 0.03 & 42 & 42 & 0 & 18.33 & 0.15 & 17.73 & 0.07 & 0.60 & 0.16 & 18.28 & 0.16 & 17.58 & 0.07 \\
\hline 393 & UGC10736 & 0.03 & 210 & 61 & -25 & 15.94 & 0.09 & 15.57 & 0.03 & 0.37 & 0.10 & 15.95 & 0.09 & 15.57 & 0.03 \\
\hline 394 & IC4662 & 0.07 & 162 & 92 & -75 & 12.43 & 0.05 & 12.35 & 0.03 & 0.08 & 0.06 & 12.44 & 0.05 & 12.35 & 0.03 \\
\hline 395 & NGC6503 & 0.03 & 528 & 178 & -57 & 13.15 & 0.05 & 12.71 & 0.03 & 0.44 & 0.06 & 13.18 & 0.05 & 12.79 & 0.03 \\
\hline 396 & ESO140-G019 & 0.08 & $\ldots$ & $\ldots$ & $\ldots$ & $\ldots$ & $\ldots$ & $\ldots$ & $\ldots$ & $\ldots$ & $\ldots$ & $\ldots$ & $\ldots$ & $\ldots$ & $\ldots$ \\
\hline 397 & IC4710 & 0.09 & $\ldots$ & $\ldots$ & $\ldots$ & $\ldots$ & $\ldots$ & $\ldots$ & $\ldots$ & $\ldots$ & $\ldots$ & $\ldots$ & $\ldots$ & $\ldots$ & $\ldots$ \\
\hline 398 & NGC6689 & 0.07 & $\ldots$ & $\ldots$ & $\ldots$ & $\ldots$ & $\ldots$ & $\ldots$ & $\ldots$ & $\ldots$ & $\ldots$ & $\ldots$ & $\ldots$ & $\ldots$ & $\ldots$ \\
\hline 399 & ESO104-G022 & 0.08 & $\ldots$ & $\ldots$ & $\ldots$ & $\ldots$ & $\ldots$ & $\ldots$ & $\ldots$ & $\ldots$ & $\ldots$ & $\ldots$ & $\ldots$ & $\ldots$ & $\ldots$ \\
\hline 400 & NGC6744 & 0.04 & 1002 & 646 & 15 & 11.14 & 0.07 & 10.69 & 0.03 & 0.45 & 0.08 & 11.12 & 0.07 & 10.71 & 0.04 \\
\hline 401 & ESO104-G044 & 0.04 & 72 & 61 & -18 & 17.28 & 0.06 & 16.64 & 0.04 & 0.64 & 0.07 & 17.26 & 0.07 & 16.58 & 0.04 \\
\hline 402 & NGC6789 & 0.07 & 60 & 46 & -35 & 16.22 & 0.05 & 15.65 & 0.03 & 0.57 & 0.06 & 16.21 & 0.05 & 15.61 & 0.03 \\
\hline 403 & ESO594-G004 & 0.12 & 132 & 96 & -85 & 14.89 & 0.12 & 14.65 & 0.03 & 0.24 & 0.12 & 14.89 & 0.12 & 14.61 & 0.03 \\
\hline 404 & IC4870 & 0.11 & 72 & 40 & -44 & 14.63 & 0.13 & 14.43 & 0.04 & 0.20 & 0.14 & 14.58 & 0.13 & 14.41 & 0.04 \\
\hline 405 & NGC6822 & 0.23 & 918 & 800 & 0 & 10.08 & 0.05 & 9.62 & 0.07 & 0.46 & 0.09 & 10.05 & 0.06 & 9.54 & 0.07 \\
\hline 406 & IC4951 & 0.04 & 204 & 36 & -4 & 15.22 & 0.05 & 14.94 & 0.04 & 0.28 & 0.06 & 15.22 & 0.05 & 14.94 & 0.04 \\
\hline 407 & UGC11583 & 0.31 & $\cdots$ & $\ldots$ & $\ldots$ & $\ldots$ & $\cdots$ & $\ldots$ & $\ldots$ & $\cdots$ & $\ldots$ & $\ldots$ & $\ldots$ & $\ldots$ & $\ldots$ \\
\hline 408 & LEDA166192 & 0.28 & $\ldots$ & $\ldots$ & $\ldots$ & $\ldots$ & $\ldots$ & $\ldots$ & $\ldots$ & $\ldots$ & $\ldots$ & $\ldots$ & $\ldots$ & $\ldots$ & $\ldots$ \\
\hline 409 & LEDA166193 & 0.45 & $\ldots$ & $\ldots$ & $\ldots$ & $\ldots$ & $\ldots$ & $\ldots$ & $\ldots$ & $\ldots$ & $\ldots$ & $\ldots$ & $\ldots$ & $\ldots$ & $\ldots$ \\
\hline 410 & NGC6946 & 0.34 & 504 & 429 & 52 & 10.29 & 0.34 & 9.70 & 0.10 & 0.59 & 0.36 & 10.30 & 0.34 & 9.70 & 0.11 \\
\hline 411 & KKR55 & 0.70 & $\ldots$ & $\ldots$ & $\ldots$ & $\ldots$ & $\ldots$ & $\ldots$ & $\ldots$ & $\ldots$ & $\ldots$ & $\ldots$ & $\ldots$ & $\ldots$ & $\ldots$ \\
\hline 412 & DDO210 & 0.05 & 96 & 48 & -80 & 16.59 & 0.05 & 16.23 & 0.04 & 0.36 & 0.07 & 16.54 & 0.05 & 16.10 & 0.11 \\
\hline 413 & KKR56 & 0.73 & $\ldots$ & $\ldots$ & $\ldots$ & $\ldots$ & $\ldots$ & $\ldots$ & $\ldots$ & $\ldots$ & $\ldots$ & $\ldots$ & $\ldots$ & $\ldots$ & $\ldots$ \\
\hline 414 & CEPHEUS1 & 0.92 & $\ldots$ & $\ldots$ & $\ldots$ & $\ldots$ & $\ldots$ & $\ldots$ & $\ldots$ & $\ldots$ & $\ldots$ & $\ldots$ & $\ldots$ & $\ldots$ & $\ldots$ \\
\hline 415 & IC5052 & 0.05 & 348 & 47 & -37 & 13.92 & 0.05 & 13.37 & 0.03 & 0.55 & 0.06 & 13.93 & 0.05 & 13.38 & 0.03 \\
\hline 416 & KKR59 & 0.88 & $\ldots$ & $\ldots$ & $\ldots$ & $\ldots$ & $\ldots$ & $\ldots$ & $\ldots$ & $\ldots$ & $\ldots$ & $\ldots$ & $\ldots$ & $\ldots$ & $\ldots$ \\
\hline 417 & KKR60 & 1.03 & $\ldots$ & $\ldots$ & $\ldots$ & $\ldots$ & $\ldots$ & $\ldots$ & $\ldots$ & $\ldots$ & $\ldots$ & $\ldots$ & $\ldots$ & $\ldots$ & $\ldots$ \\
\hline 418 & NGC7064 & 0.01 & 282 & 45 & -89 & 14.15 & 0.05 & 13.89 & 0.03 & 0.26 & 0.06 & 14.15 & 0.05 & 13.89 & 0.03 \\
\hline 419 & NGC7090 & 0.02 & 552 & 97 & -53 & 14.06 & 0.06 & 13.50 & 0.06 & 0.56 & 0.09 & 14.10 & 0.06 & 13.53 & 0.06 \\
\hline 420 & IC5152 & 0.03 & 228 & 140 & -80 & 12.33 & 0.05 & 11.99 & 0.03 & 0.34 & 0.06 & 12.33 & 0.05 & 11.98 & 0.03 \\
\hline 421 & UGC11891 & 0.35 & $\ldots$ & $\ldots$ & $\ldots$ & $\ldots$ & $\ldots$ & $\ldots$ & $\ldots$ & $\ldots$ & $\ldots$ & $\ldots$ & $\ldots$ & $\ldots$ & $\ldots$ \\
\hline 422 & ESO238-G005 & 0.01 & 216 & 97 & 5 & 16.44 & 0.05 & 16.23 & 0.04 & 0.21 & 0.07 & 16.44 & 0.05 & 16.23 & 0.04 \\
\hline 423 & IC5256 & 0.03 & 78 & 36 & 19 & 16.72 & 0.05 & 16.32 & 0.03 & 0.40 & 0.06 & 16.72 & 0.05 & 16.32 & 0.03 \\
\hline 424 & NGC7640 & 0.11 & $\ldots$ & $\ldots$ & $\ldots$ & $\ldots$ & $\ldots$ & $\ldots$ & $\ldots$ & $\ldots$ & $\ldots$ & $\ldots$ & $\ldots$ & $\ldots$ & $\ldots$ \\
\hline 425 & UGC12588 & 0.15 & $\ldots$ & $\ldots$ & $\ldots$ & $\ldots$ & $\ldots$ & $\ldots$ & $\ldots$ & $\ldots$ & $\ldots$ & $\ldots$ & $\ldots$ & $\ldots$ & $\ldots$ \\
\hline 426 & UGCA438 & 0.01 & 84 & 67 & 0 & 15.31 & 0.05 & 15.26 & 0.03 & 0.05 & 0.06 & 15.31 & 0.05 & 15.25 & 0.03 \\
\hline 427 & ESO347-G017 & 0.02 & 204 & 44 & -86 & 15.61 & 0.05 & 15.31 & 0.03 & 0.30 & 0.06 & 15.61 & 0.05 & 15.32 & 0.03 \\
\hline 428 & UGC12613 & 0.07 & 216 & 117 & -60 & 15.71 & 0.06 & 14.94 & 0.06 & 0.77 & 0.08 & 15.73 & 0.06 & 14.93 & 0.06 \\
\hline 429 & UGC12632 & 0.14 & 156 & 128 & 20 & 14.66 & 0.07 & 14.20 & 0.11 & 0.46 & 0.13 & 14.56 & 0.07 & 14.09 & 0.12 \\
\hline 430 & IC5332 & 0.02 & 462 & 367 & 17 & 12.55 & 0.05 & 12.35 & 0.03 & 0.20 & 0.06 & 12.48 & 0.07 & 12.27 & 0.06 \\
\hline 431 & NGC7713 & 0.02 & $\ldots$ & $\ldots$ & $\ldots$ & $\ldots$ & $\ldots$ & $\ldots$ & $\ldots$ & $\ldots$ & $\ldots$ & $\ldots$ & $\ldots$ & $\ldots$ & $\ldots$ \\
\hline 432 & UGC12713 & 0.06 & 72 & 43 & 65 & 16.43 & 0.06 & 16.13 & 0.04 & 0.30 & 0.07 & 16.43 & 0.06 & 16.12 & 0.04 \\
\hline 433 & UGCA442 & 0.02 & 258 & 37 & 48 & 14.83 & 0.05 & 14.67 & 0.04 & 0.16 & 0.06 & 14.83 & 0.05 & 14.67 & 0.04 \\
\hline
\end{tabular}


Table 2

(Continued)

\begin{tabular}{|c|c|c|c|c|c|c|c|c|c|c|c|c|c|c|c|}
\hline \multirow{2}{*}{$\begin{array}{l}\text { No. } \\
\text { (1) }\end{array}$} & \multirow{2}{*}{$\begin{array}{c}\text { Galaxy Name } \\
\text { (2) }\end{array}$} & \multirow{2}{*}{$\begin{array}{c}E(B-V) \\
(3)\end{array}$} & \multirow{2}{*}{$\begin{array}{l}a \\
(") \\
(4)\end{array}$} & \multirow{2}{*}{$\begin{array}{l}b \\
\left({ }^{\prime \prime}\right) \\
(5)\end{array}$} & \multirow{2}{*}{$\begin{array}{l}\text { P.A. } \\
\text { (deg) } \\
(6)\end{array}$} & \multicolumn{2}{|c|}{$\begin{array}{c}\text { FUV }_{\text {ap }} \\
\text { (mag) }\end{array}$} & \multicolumn{2}{|c|}{$\begin{array}{c}\mathrm{NUV}_{\mathrm{ap}} \\
\text { (mag) }\end{array}$} & \multicolumn{2}{|c|}{$\begin{array}{c}(\mathrm{FUV}- \\
\mathrm{NUV})_{\mathrm{ap}} \\
(\mathrm{mag})\end{array}$} & \multicolumn{2}{|c|}{$\begin{array}{c}\mathrm{FUV}_{\text {asym }} \\
(\mathrm{mag})\end{array}$} & \multicolumn{2}{|c|}{$\begin{array}{c}\mathrm{NUV}_{\text {asym }} \\
(\mathrm{mag})\end{array}$} \\
\hline & & & & & & (7) & (8) & (9) & $(10)$ & (11) & (12) & (13) & (14) & (15) & (16) \\
\hline 434 & ESO348-G009 & 0.01 & 162 & 68 & 83 & 16.27 & 0.05 & 16.05 & 0.04 & 0.22 & 0.06 & 16.25 & 0.05 & 16.01 & 0.04 \\
\hline 435 & ESO149-G003 & 0.01 & 162 & 30 & -32 & 15.66 & 0.05 & 15.59 & 0.03 & 0.07 & 0.06 & 15.66 & 0.05 & 15.59 & 0.03 \\
\hline 436 & NGC7793 & 0.02 & 618 & 418 & -82 & 11.12 & 0.05 & 10.97 & 0.03 & 0.15 & 0.06 & 11.13 & 0.05 & 10.97 & 0.03 \\
\hline 1 & ESO410-G005 & 0.01 & 108 & 83 & 54 & 18.26 & 0.09 & 17.20 & 0.04 & 1.06 & 0.10 & 18.29 & 0.11 & 17.20 & 0.05 \\
\hline 2 & SCULPTOR-DE1 & 0.01 & 102 & 79 & -10 & $(23.60)$ & $\ldots$ & 18.45 & 0.20 & $\ldots$ & $\ldots$ & $\ldots$ & $\ldots$ & 17.82 & 0.92 \\
\hline 3 & ESO294-G010 & 0.01 & 72 & 46 & 6 & 18.12 & 0.07 & 17.57 & 0.04 & 0.55 & 0.08 & 18.02 & 0.12 & 17.43 & 0.04 \\
\hline 4 & ESO540-G030 & 0.02 & 108 & 81 & -70 & 19.10 & 0.11 & 18.08 & 0.10 & 1.02 & 0.15 & 19.28 & 0.15 & 18.30 & 0.28 \\
\hline 5 & ESO540-G032 & 0.02 & 30 & 18 & 0 & 19.58 & 0.08 & 19.07 & 0.05 & 0.51 & 0.09 & 19.53 & 0.10 & 18.66 & 0.07 \\
\hline 6 & UGC521 & 0.06 & 60 & 40 & 50 & 16.03 & 0.05 & 16.05 & 0.03 & -0.02 & 0.06 & 16.02 & 0.05 & 16.04 & 0.03 \\
\hline 7 & NGC0404 & 0.06 & 396 & 396 & 0 & 14.94 & 0.26 & 13.69 & 0.17 & 1.25 & 0.32 & 14.94 & 0.43 & 13.68 & 0.54 \\
\hline 8 & IC2049 & 0.02 & 60 & 54 & 3 & 16.46 & 0.05 & 16.11 & 0.04 & 0.35 & 0.06 & 16.45 & 0.05 & 16.10 & 0.04 \\
\hline 9 & UGCA133 & 0.04 & $\ldots$ & $\ldots$ & $\ldots$ & $(23.07)$ & $\ldots$ & $(23.07)$ & $\ldots$ & $\ldots$ & $\ldots$ & $\ldots$ & $\ldots$ & $\ldots$ & $\ldots$ \\
\hline 10 & F8D1 & 0.10 & 100 & 100 & 0 & 24.37 & 2.74 & 17.12 & 0.13 & $\ldots$ & $\ldots$ & $\ldots$ & $\ldots$ & $\ldots$ & $\ldots$ \\
\hline 11 & FM2000_1 & 0.08 & 54 & 48 & 0 & $(23.95)$ & $\ldots$ & 19.88 & 0.07 & $\ldots$ & $\ldots$ & $\ldots$ & $\ldots$ & 19.68 & 0.10 \\
\hline 12 & LEDA166101 & 0.14 & 102 & 76 & 20 & $(22.24)$ & $\ldots$ & 18.00 & 0.10 & $\ldots$ & $\ldots$ & $\ldots$ & $\ldots$ & 18.10 & 0.99 \\
\hline 13 & ARPSLOOP & 0.09 & 78 & 69 & 0 & 18.49 & 0.09 & 17.97 & 0.05 & 0.52 & 0.10 & 17.31 & 0.73 & 17.76 & 0.75 \\
\hline 14 & KKH057 & 0.02 & 24 & 20 & 10 & $(23.24)$ & $\ldots$ & 20.87 & 0.14 & $\ldots$ & $\ldots$ & $\ldots$ & $\ldots$ & 20.31 & 0.23 \\
\hline 15 & AM1001-270 & 0.08 & 114 & 86 & -20 & 17.11 & 0.35 & 16.44 & 0.14 & 0.67 & 0.38 & 16.44 & 0.50 & 16.07 & 0.17 \\
\hline 16 & $\mathrm{BK} 05 \mathrm{~N}$ & 0.06 & 42 & 32 & -45 & $(23.18)$ & $\ldots$ & 20.31 & 0.12 & $\ldots$ & $\ldots$ & $\ldots$ & $\ldots$ & 19.69 & 0.41 \\
\hline 17 & UGC05442 & 0.05 & 84 & 44 & 23 & $(23.42)$ & $\ldots$ & 18.39 & 0.05 & $\ldots$ & $\ldots$ & $\ldots$ & $\ldots$ & 17.80 & 0.09 \\
\hline 18 & IKN & 0.06 & $\ldots$ & $\ldots$ & $\ldots$ & $(22.90)$ & $\ldots$ & $(23.06)$ & $\ldots$ & $\ldots$ & $\ldots$ & $\ldots$ & $\ldots$ & $\ldots$ & $\ldots$ \\
\hline 19 & HS98_117 & 0.12 & $\ldots$ & $\ldots$ & $\ldots$ & $(20.82)$ & $\ldots$ & $(20.98)$ & $\ldots$ & $\ldots$ & $\ldots$ & $\ldots$ & $\ldots$ & $\ldots$ & $\ldots$ \\
\hline 20 & DDO078 & 0.02 & $\ldots$ & $\ldots$ & $\ldots$ & $(22.01)$ & $\ldots$ & $(22.05)$ & $\ldots$ & $\ldots$ & $\ldots$ & $\ldots$ & $\ldots$ & $\ldots$ & $\ldots$ \\
\hline 21 & $\mathrm{BK} 06 \mathrm{~N}$ & 0.01 & $\ldots$ & $\ldots$ & $\ldots$ & $(23.39)$ & $\ldots$ & $(23.30)$ & $\ldots$ & $\ldots$ & $\ldots$ & $\ldots$ & $\ldots$ & $\ldots$ & $\ldots$ \\
\hline 22 & UGC6782 & 0.03 & 144 & 144 & 0 & 16.85 & 0.16 & 16.48 & 0.09 & 0.37 & 0.18 & 16.87 & 0.16 & 16.48 & 0.09 \\
\hline 23 & ESO321-G014 & 0.09 & 102 & 44 & 20 & 16.41 & 0.14 & 16.12 & 0.04 & 0.29 & 0.14 & 16.42 & 0.15 & 16.08 & 0.05 \\
\hline 24 & UGC7321 & 0.03 & 246 & 18 & 82 & 16.10 & 0.05 & 15.46 & 0.03 & 0.64 & 0.06 & 16.09 & 0.05 & 15.44 & 0.03 \\
\hline 25 & ККH086 & 0.03 & 72 & 51 & 0 & 19.27 & 0.12 & 18.54 & 0.11 & 0.73 & 0.17 & 19.46 & 0.16 & 18.58 & 0.13 \\
\hline 26 & KKR25 & 0.01 & 66 & 36 & 20 & $(23.45)$ & $\ldots$ & 19.11 & 0.06 & $\ldots$ & $\ldots$ & $\ldots$ & $\ldots$ & 18.91 & 0.11 \\
\hline 27 & ККH098 & 0.12 & 54 & 29 & -5 & 17.20 & 0.06 & 17.00 & 0.04 & 0.20 & 0.07 & 17.19 & 0.07 & 16.94 & 0.05 \\
\hline
\end{tabular}

(This table is also available in a machine-readable form in the online journal.)

growth curve in each GALEX UV band is computed. A linear fit to the cumulative magnitude versus the cumulative-magnitude gradient is performed, and the $y$-intercept of the fit is adopted as the asymptotic magnitude. The uncertainty in the fit is also included in the reported errors. This procedure is relatively standard at optical wavelengths (see, e.g., Cairós et al. 2001) and has been shown to be accurate for the determination of total magnitudes in GALEX UV imaging of nearby galaxies (Gil de Paz et al. 2007).

Aperture fluxes are measured within the outermost elliptical annulus where both FUV and NUV surface photometry can be performed. We define this annulus as the one beyond which either the flux error becomes larger than 0.8 mag or where the intensity falls below that of the sky background in both FUV and NUV bands. These matched aperture fluxes are appropriate for computing the integrated color.

Finally, fluxes are also measured in the apertures used for the Local Volume Legacy Spitzer imaging in Dale et al. (2009) to provide a complete set of matched UV-to-IR aperture photometry as mentioned above.

The results of our photometry, on the AB magnitude scale, are presented in Table 2 as follows.

Column 1: index number, repeated from Table 1.

Column 2: galaxy name, repeated from Table 1.

Column 3: $E(B-V)$ based on the maps of Schlegel et al. (1998).
Columns 4-6: semimajor axis, semiminor axis, and position angle of the outermost elliptical annulus where both FUV and NUV surface photometry can be performed. We define this as the distance beyond which the annular flux error becomes larger than $0.8 \mathrm{mag}$, or where the intensity falls below that of the sky background in both FUV and NUV bands, whichever occurs first. The position angle is measured east of north as usual.

Columns 7-10: FUV and NUV magnitude and error, measured in the common elliptical aperture described by the preceding columns. Corrections for Milky Way (MW) foreground extinction have been applied using the $E(B-V)$ values listed here, and the Cardelli et al. (1989) extinction law with $R_{V}=3.1$. That is, $A_{\mathrm{FUV}}=7.9 E(B-V)$ and $A_{\mathrm{FUV}}=8.0 E(B-V)$. For non-detections, $3 \sigma$ point source upper limits are listed, enclosed in parenthesis. The quoted errors include the photometric uncertainty within the aperture and the error in the zero point $(0.05$ and $0.03 m_{\mathrm{AB}}$ in the FUV and NUV, respectively; Morrissey et al. 2007).

Columns 11 and 12: FUV - NUV color and error, corrected for MW extinction as described previously.

Columns 13-16: FUV and NUV asymptotic magnitude and error, measured from a growth curve which is computed using fixed central coordinates, ellipticity, and position angle as described above, and corrected for MW extinction. Here, the quoted errors include the photometric uncertainty, the error 
Table 3

GALEX Photometry in Spitzer Local Volume Legacy Apertures

\begin{tabular}{|c|c|c|c|c|c|c|c|c|}
\hline \multirow{2}{*}{$\begin{array}{l}\text { No. } \\
\text { (1) }\end{array}$} & \multirow{2}{*}{$\begin{array}{c}\text { Galaxy Name } \\
\text { (2) }\end{array}$} & \multirow{2}{*}{$\begin{array}{c}a \\
(") \\
(3) \\
\end{array}$} & \multirow{2}{*}{$\begin{array}{l}b \\
(") \\
(4)\end{array}$} & \multirow{2}{*}{$\begin{array}{l}\text { P.A. } \\
(\mathrm{deg}) \\
(5)\end{array}$} & \multicolumn{2}{|c|}{$\begin{array}{c}\mathrm{FUV}_{\text {LocalVolumeLegacy }} \\
\text { (mag) }\end{array}$} & \multicolumn{2}{|c|}{$\begin{array}{c}\mathrm{NUV}_{\text {LocalVolumeLegacy }} \\
\text { (mag) }\end{array}$} \\
\hline & & & & & (6) & (7) & $(8)$ & (9) \\
\hline 2 & WLM & 336 & 170 & 0 & 12.52 & 0.05 & 12.40 & 0.03 \\
\hline 5 & $\mathrm{NGC} 24$ & 150 & 108 & 225 & 14.08 & 0.05 & 13.81 & 0.03 \\
\hline 6 & NGC45 & 288 & 228 & 336 & 12.58 & 0.05 & 12.39 & 0.03 \\
\hline 7 & NGC55 & 1126 & 357 & 106 & 10.16 & 0.05 & 9.86 & 0.03 \\
\hline 8 & NGC59 & 128 & 90 & 302 & 16.06 & 0.06 & 15.31 & 0.03 \\
\hline 14 & IC1574 & 101 & 62 & 0 & 16.56 & 0.05 & 16.08 & 0.03 \\
\hline 15 & NGC247 & 738 & 290 & 352 & 11.38 & 0.05 & 11.16 & 0.03 \\
\hline 16 & NGC253 & 1025 & 404 & 50 & 11.15 & 0.05 & 10.62 & 0.03 \\
\hline 17 & UGCA15 & 75 & 39 & 28 & 16.78 & 0.05 & 16.60 & 0.04 \\
\hline 18 & SMC & $\ldots$ & $\ldots$ & $\ldots$ & $\ldots$ & $\ldots$ & $\ldots$ & $\ldots$ \\
\hline 19 & NGC300 & 754 & 564 & 114 & 10.23 & 0.05 & 10.10 & 0.03 \\
\hline 21 & UGC668 & 341 & 274 & 60 & 11.70 & 0.05 & 11.67 & 0.03 \\
\hline 22 & UGC685 & 90 & 74 & 122 & 16.02 & 0.06 & 15.61 & 0.04 \\
\hline 23 & UGC695 & 64 & 54 & 0 & 16.64 & 0.05 & 16.27 & 0.03 \\
\hline 24 & UGC891 & 97 & 59 & 42 & 16.30 & 0.05 & 16.07 & 0.03 \\
\hline 25 & UGC1056 & 62 & 58 & 0 & 16.63 & 0.05 & 16.26 & 0.03 \\
\hline 26 & UGC1104 & 83 & 52 & 0 & 15.60 & 0.05 & 15.41 & 0.03 \\
\hline 27 & NGC598 & 2226 & 1381 & 12 & 8.00 & 0.05 & 7.72 & 0.03 \\
\hline 28 & NGC625 & 250 & 128 & 90 & 13.72 & 0.05 & 13.36 & 0.03 \\
\hline 29 & NGC628 & 440 & 404 & 90 & 11.68 & 0.05 & 11.40 & 0.03 \\
\hline 30 & UGC1176 & 101 & 84 & 25 & 15.79 & 0.06 & 15.62 & 0.05 \\
\hline 32 & ESO245-G005 & 179 & 126 & 318 & 13.66 & 0.05 & 13.62 & 0.03 \\
\hline 33 & UGC1249 & 234 & 102 & 331 & 13.45 & 0.05 & 13.28 & 0.03 \\
\hline 34 & NGC672 & 278 & 180 & 67 & 13.18 & 0.05 & 12.83 & 0.03 \\
\hline 36 & ESO245-G007 & 144 & 120 & 0 & 16.25 & 0.10 & 15.52 & 0.04 \\
\hline 37 & NGC784 & 240 & 96 & 3 & 13.91 & 0.05 & 13.51 & 0.03 \\
\hline 39 & NGC855 & 95 & 86 & 68 & 15.88 & 0.11 & 15.22 & 0.04 \\
\hline 50 & ESO115-G021 & 200 & 56 & 221 & 14.65 & 0.05 & 14.39 & 0.03 \\
\hline 55 & ESO154-G023 & 243 & 124 & 39 & 13.78 & 0.05 & 13.52 & 0.04 \\
\hline 59 & NGC1291 & 420 & 402 & 0 & 14.22 & 0.11 & 13.38 & 0.05 \\
\hline 60 & NGC1313 & 448 & 347 & 338 & 10.62 & 0.07 & 10.45 & 0.04 \\
\hline 61 & NGC1311 & 150 & 70 & 36 & 14.83 & 0.05 & 14.42 & 0.03 \\
\hline 64 & UGC2716 & 87 & 62 & 90 & 15.91 & 0.06 & 15.43 & 0.05 \\
\hline 65 & IC1959 & 126 & 57 & 330 & 14.37 & 0.05 & 14.25 & 0.03 \\
\hline 69 & NGC1487 & 215 & 130 & 70 & 13.44 & 0.05 & 13.25 & 0.03 \\
\hline 72 & NGC1510 & 63 & 61 & 0 & 15.09 & 0.05 & 14.87 & 0.03 \\
\hline 73 & NGC1512 & 501 & 464 & 83 & 13.44 & 0.05 & 13.21 & 0.05 \\
\hline 74 & NGC1522 & 76 & 50 & 37 & 15.17 & 0.05 & 15.03 & 0.03 \\
\hline 76 & ESO483-G013 & 102 & 74 & 322 & 15.77 & 0.05 & 15.42 & 0.03 \\
\hline 83 & ESO158-G003 & 100 & 86 & 0 & 15.47 & 0.05 & 15.21 & 0.03 \\
\hline 85 & ESO119-G016 & 110 & 58 & 26 & 16.03 & 0.06 & 15.76 & 0.03 \\
\hline 86 & NGC1705 & 84 & 60 & 220 & 13.36 & 0.05 & 13.42 & 0.03 \\
\hline 88 & NGC1744 & 330 & 158 & 349 & 13.07 & 0.05 & 12.90 & 0.03 \\
\hline 89 & NGC1796 & 128 & 102 & 99 & 14.91 & 0.05 & 14.40 & 0.03 \\
\hline 90 & ESO486-G021 & 58 & 50 & 90 & 15.30 & 0.05 & 15.08 & 0.03 \\
\hline 91 & MCG-05-13-004 & $\ldots$ & $\ldots$ & $\ldots$ & $\ldots$ & $\ldots$ & $\ldots$ & $\ldots$ \\
\hline 92 & NGC1800 & 114 & 82 & 107 & 14.60 & 0.05 & 14.43 & 0.03 \\
\hline 96 & UGCA106 & 162 & 122 & 14 & 14.06 & 0.05 & 13.94 & 0.05 \\
\hline 98 & LMC & $\ldots$ & $\ldots$ & $\ldots$ & $\ldots$ & $\ldots$ & $\ldots$ & $\ldots$ \\
\hline 112 & KKH37 & 52 & 40 & 90 & 18.29 & 0.08 & 17.74 & 0.04 \\
\hline 119 & NGC2366 & 308 & 158 & 31 & 12.47 & 0.05 & 12.39 & 0.03 \\
\hline 123 & NGC2403 & 756 & 465 & 304 & 10.33 & 0.05 & 10.13 & 0.03 \\
\hline 128 & NGC2500 & 137 & 117 & 75 & 13.48 & 0.05 & 13.34 & 0.03 \\
\hline 129 & NGC2537 & 106 & 100 & 0 & 13.89 & 0.05 & 13.69 & 0.03 \\
\hline 130 & UGC4278 & 160 & 40 & 351 & 14.44 & 0.05 & 14.21 & 0.03 \\
\hline 131 & UGC4305 & 278 & 232 & 60 & 12.35 & 0.05 & 12.25 & 0.03 \\
\hline 132 & NGC2552 & 156 & 102 & 54 & 14.27 & 0.05 & 14.09 & 0.03 \\
\hline 133 & M81dwA & 39 & 39 & 90 & 17.35 & 0.05 & 17.36 & 0.03 \\
\hline 135 & UGC4426 & 103 & 72 & 10 & 16.76 & 0.06 & 16.53 & 0.03 \\
\hline 136 & UGC4459 & 67 & 56 & 120 & 15.32 & 0.05 & 15.38 & 0.03 \\
\hline 138 & UGC4483 & 47 & 29 & 0 & 15.75 & 0.05 & 15.80 & 0.03 \\
\hline 139 & NGC2683 & 411 & 210 & 41 & 14.09 & 0.05 & 13.45 & 0.09 \\
\hline 140 & UGC4704 & 152 & 54 & 296 & 15.83 & 0.07 & 15.60 & 0.03 \\
\hline
\end{tabular}


Table 3

(Continued)

\begin{tabular}{|c|c|c|c|c|c|c|c|c|}
\hline \multirow{2}{*}{$\begin{array}{l}\text { No. } \\
\text { (1) } \\
\end{array}$} & \multirow{2}{*}{$\begin{array}{c}\text { Galaxy Name } \\
\text { (2) }\end{array}$} & \multirow{2}{*}{$\begin{array}{l}a \\
(") \\
(3)\end{array}$} & \multirow{2}{*}{$\begin{array}{c}b \\
\left({ }^{\prime \prime}\right) \\
(4) \\
\end{array}$} & \multirow{2}{*}{$\begin{array}{c}\text { P.A. } \\
(\mathrm{deg}) \\
(5)\end{array}$} & \multicolumn{2}{|c|}{$\begin{array}{c}\text { FUVV }_{\text {LocalVolumeLegacy }} \\
\text { (mag) }\end{array}$} & \multicolumn{2}{|c|}{$\begin{array}{c}\text { NUV }_{\text {LocalVolumeLegacy }} \\
\text { (mag) }\end{array}$} \\
\hline & & & & & (6) & (7) & $(8)$ & (9) \\
\hline 142 & UGC4787 & 106 & 54 & 5 & 15.99 & 0.05 & 15.57 & 0.04 \\
\hline 150 & UGC4998 & 94 & 81 & 71 & 17.98 & 0.18 & 17.15 & 0.04 \\
\hline 152 & NGC2903 & 412 & 230 & 17 & 12.31 & 0.05 & 11.76 & 0.03 \\
\hline 153 & UGC5076 & 82 & 70 & 90 & $\ldots$ & $\ldots$ & $\ldots$ & $\ldots$ \\
\hline 154 & CGCG035-007 & 63 & 48 & 63 & 17.13 & 0.06 & 16.72 & 0.03 \\
\hline 157 & UGC5139 & 132 & 110 & 63 & 14.74 & 0.06 & 14.71 & 0.03 \\
\hline 158 & IC559 & 68 & 62 & 63 & 16.44 & 0.05 & 16.06 & 0.03 \\
\hline 160 & NGC2976 & 228 & 156 & 322 & 13.07 & 0.08 & 12.73 & 0.03 \\
\hline 162 & UGC5272 & 98 & 48 & 112 & 14.96 & 0.05 & 14.88 & 0.03 \\
\hline 163 & UGC5288 & 82 & 70 & 331 & 15.73 & 0.05 & 15.52 & 0.03 \\
\hline 165 & BK3N & 20 & 20 & 90 & 18.84 & 0.06 & 18.78 & 0.04 \\
\hline 166 & NGC3031 & 814 & 562 & 154 & 10.77 & 0.05 & 10.47 & 0.03 \\
\hline 167 & NGC3034 & 349 & 290 & 65 & 12.56 & 0.09 & 11.47 & 0.03 \\
\hline 168 & UGC5340 & 87 & 50 & 10 & 15.16 & 0.05 & 15.18 & 0.03 \\
\hline 169 & KDG61 & 107 & 60 & 49 & 21.53 & 1.54 & 18.44 & 0.12 \\
\hline 170 & UGC5336 & 124 & 90 & 220 & 15.04 & 0.06 & 14.90 & 0.05 \\
\hline 173 & UGC5364 & 156 & 96 & 67 & 14.62 & 0.05 & 14.35 & 0.03 \\
\hline 174 & UGC5373 & 166 & 134 & 90 & 13.72 & 0.05 & 13.52 & 0.04 \\
\hline 175 & UGCA193 & 142 & 34 & 14 & 16.27 & 0.05 & 15.94 & 0.04 \\
\hline 176 & NGC3109 & 798 & 174 & 91 & 11.37 & 0.12 & 11.11 & 0.07 \\
\hline 177 & NGC3077 & 244 & 218 & 64 & $\ldots$ & & $\ldots$ & $\ldots$ \\
\hline 181 & UGC5428 & 98 & 84 & 90 & 17.93 & 0.08 & $\ldots$ & $\ldots$ \\
\hline 183 & UGC5423 & 54 & 35 & 140 & 16.60 & 0.05 & 16.43 & 0.03 \\
\hline 187 & UGC5456 & 80 & 60 & 322 & 14.95 & 0.05 & 14.62 & 0.03 \\
\hline 190 & SextansA & 204 & 158 & 35 & 12.58 & 0.05 & 12.55 & 0.03 \\
\hline 192 & NGC3239 & 158 & 136 & 63 & 12.81 & 0.05 & 12.59 & 0.03 \\
\hline 193 & UGC5672 & 144 & 52 & 340 & 17.14 & 0.05 & 16.48 & 0.03 \\
\hline 194 & UGC5666 & 432 & 243 & 59 & 12.15 & 0.06 & 12.10 & 0.04 \\
\hline 195 & UGC5692 & 153 & 106 & 0 & 16.39 & 0.06 & 15.65 & 0.03 \\
\hline 196 & NGC3274 & 174 & 71 & 110 & 14.26 & 0.05 & 14.05 & 0.03 \\
\hline 199 & NGC3299 & 132 & 104 & 0 & 16.26 & 0.08 & 15.64 & 0.06 \\
\hline 200 & UGC5764 & 75 & 42 & 44 & 16.25 & 0.05 & 16.16 & 0.03 \\
\hline 201 & UGC5797 & 70 & 69 & 0 & 16.85 & 0.05 & 16.42 & 0.03 \\
\hline 203 & UGC5829 & 142 & 126 & 20 & 14.08 & 0.05 & 14.00 & 0.03 \\
\hline 204 & NGC3344 & 257 & 224 & 330 & 12.44 & 0.05 & 12.11 & 0.03 \\
\hline 205 & NGC3351 & 293 & 228 & 10 & 13.27 & 0.05 & 12.73 & 0.03 \\
\hline 207 & NGC3368 & 256 & 174 & 346 & 14.04 & 0.06 & 13.41 & 0.03 \\
\hline 208 & UGC5889 & 101 & 95 & 0 & 16.30 & 0.06 & 15.88 & 0.05 \\
\hline 210 & UGC5923 & 48 & 30 & 353 & 17.24 & 0.05 & 16.71 & 0.03 \\
\hline 211 & UGC5918 & 70 & 56 & 65 & 16.82 & 0.13 & 16.56 & 0.04 \\
\hline 214 & NGC3432 & 238 & 94 & 38 & 13.26 & 0.05 & 12.84 & 0.03 \\
\hline 215 & KDG73 & 63 & 50 & 345 & 18.83 & 0.12 & 18.41 & 0.18 \\
\hline 217 & NGC3486 & 248 & 194 & 83 & 12.50 & 0.05 & 12.35 & 0.03 \\
\hline 219 & NGC3510 & 155 & 68 & 345 & 14.61 & 0.05 & 14.33 & 0.03 \\
\hline 222 & NGC3521 & 384 & 247 & 343 & 13.05 & 0.06 & 12.28 & 0.04 \\
\hline 225 & NGC3593 & 186 & 106 & 86 & 16.77 & 0.25 & 15.23 & 0.07 \\
\hline 229 & NGC3623 & 332 & 166 & 352 & 14.95 & 0.07 & 13.91 & 0.07 \\
\hline 230 & NGC3627 & 373 & 244 & 347 & 12.65 & 0.05 & 11.98 & 0.03 \\
\hline 231 & NGC3628 & 520 & 310 & 102 & 14.23 & 0.09 & 13.18 & 0.03 \\
\hline 235 & UGC6457 & 75 & 56 & 19 & 16.34 & 0.06 & 15.97 & 0.03 \\
\hline 237 & UGC6541 & 62 & 44 & 316 & 15.38 & 0.05 & 15.20 & 0.03 \\
\hline 238 & NGC3738 & 110 & 88 & 343 & 13.76 & 0.05 & 13.45 & 0.03 \\
\hline 239 & NGC3741 & 100 & 54 & 10 & 15.17 & 0.05 & 15.03 & 0.03 \\
\hline 241 & UGC6817 & 135 & 72 & 65 & 14.92 & 0.05 & 14.75 & 0.03 \\
\hline 242 & UGC6900 & 102 & 72 & 107 & 17.40 & 0.06 & 16.88 & 0.05 \\
\hline 244 & NGC4020 & 111 & 68 & 18 & 15.20 & 0.05 & 14.83 & 0.03 \\
\hline 246 & NGC4068 & 128 & 89 & 22 & 14.30 & 0.05 & 14.08 & 0.03 \\
\hline 247 & NGC4080 & 76 & 66 & 312 & 16.08 & 0.05 & 15.68 & 0.03 \\
\hline 248 & NGC4096 & 278 & 122 & 18 & 14.09 & 0.05 & 13.60 & 0.03 \\
\hline 250 & NGC4144 & 218 & 94 & 103 & 13.95 & 0.05 & 13.57 & 0.03 \\
\hline 251 & NGC4163 & 106 & 82 & 4 & 15.35 & 0.05 & 14.96 & 0.03 \\
\hline 252 & NGC4190 & 105 & 82 & 45 & 14.77 & 0.05 & 14.32 & 0.03 \\
\hline 253 & UGC7242 & 78 & 42 & 0 & 16.07 & 0.05 & 15.55 & 0.04 \\
\hline
\end{tabular}


Table 3

(Continued)

\begin{tabular}{|c|c|c|c|c|c|c|c|c|}
\hline \multirow{2}{*}{$\begin{array}{l}\text { No. } \\
\text { (1) }\end{array}$} & \multirow{2}{*}{$\begin{array}{c}\text { Galaxy Name } \\
\text { (2) }\end{array}$} & \multirow{2}{*}{$\begin{array}{c}a \\
(") \\
(3)\end{array}$} & \multirow{2}{*}{$\begin{array}{c}b \\
(") \\
(4)\end{array}$} & \multirow{2}{*}{$\begin{array}{l}\text { P.A. } \\
\text { (deg) } \\
(5)\end{array}$} & \multicolumn{2}{|c|}{$\begin{array}{c}\text { FUV }_{\text {LocalVolumeLegacy }} \\
\text { (mag) }\end{array}$} & \multicolumn{2}{|c|}{$\begin{array}{c}\text { NUV }_{\text {LocalVolumeLegacy }} \\
\text { (mag) }\end{array}$} \\
\hline & & & & & (6) & (7) & (8) & (9) \\
\hline 254 & UGCA276 & 80 & 72 & 301 & 16.07 & 0.05 & 18.70 & 0.07 \\
\hline 256 & UGC7267 & 94 & 49 & 45 & 16.29 & 0.05 & 15.94 & 0.03 \\
\hline 258 & NGC4214 & 312 & 284 & 0 & 11.50 & 0.05 & 11.28 & 0.03 \\
\hline 259 & CGCG269-049 & 47 & 34 & 319 & 16.63 & 0.05 & 16.39 & 0.03 \\
\hline 261 & NGC4236 & 620 & 184 & 342 & 11.81 & 0.05 & 11.63 & 0.03 \\
\hline 262 & NGC4244 & 591 & 121 & 47 & 12.67 & 0.05 & 12.20 & 0.03 \\
\hline 263 & NGC4242 & 205 & 156 & 28 & 13.88 & 0.05 & 13.54 & 0.03 \\
\hline 264 & NGC4248 & 126 & 85 & 107 & 16.82 & 0.05 & 15.83 & 0.04 \\
\hline 266 & NGC4258 & 621 & 266 & 332 & 11.85 & 0.05 & 11.43 & 0.03 \\
\hline 268 & ISZ399 & 66 & 51 & 314 & $\ldots$ & $\ldots$ & $\ldots$ & $\ldots$ \\
\hline 269 & NGC4288 & 95 & 82 & 139 & 14.62 & 0.05 & 14.41 & 0.03 \\
\hline 270 & UGC7408 & 110 & 96 & 90 & 16.11 & 0.05 & 15.50 & 0.03 \\
\hline 271 & UGC7490 & 113 & 110 & 0 & $\ldots$ & $\ldots$ & $\ldots$ & $\ldots$ \\
\hline 273 & NGC4395 & 504 & 395 & 328 & 11.64 & 0.05 & 11.52 & 0.03 \\
\hline 274 & UGCA281 & 44 & 36 & 81 & 15.18 & 0.05 & 15.18 & 0.03 \\
\hline 275 & UGC7559 & 120 & 66 & 307 & 15.15 & 0.05 & 15.06 & 0.03 \\
\hline 276 & UGC7577 & 135 & 90 & 301 & 14.84 & 0.05 & 14.54 & 0.03 \\
\hline 279 & NGC4449 & 236 & 177 & 57 & 10.86 & 0.05 & 10.81 & 0.03 \\
\hline 280 & UGC7599 & 60 & 36 & 306 & 16.13 & 0.05 & 16.03 & 0.03 \\
\hline 281 & UGC7605 & 70 & 43 & 17 & 15.82 & 0.05 & 15.67 & 0.03 \\
\hline 282 & NGC4455 & 100 & 45 & 198 & 14.46 & 0.05 & 14.19 & 0.03 \\
\hline 283 & UGC7608 & 105 & 104 & 0 & 14.83 & 0.05 & 14.77 & 0.03 \\
\hline 284 & NGC4460 & 169 & 74 & 37 & 15.52 & 0.06 & 14.77 & 0.03 \\
\hline 286 & UGC7639 & 116 & 70 & 334 & 16.13 & 0.05 & 15.71 & 0.03 \\
\hline 288 & NGC4485 & 90 & 67 & 343 & 13.61 & 0.05 & 13.39 & 0.03 \\
\hline 289 & NGC4490 & 209 & 116 & 121 & 12.03 & 0.05 & 11.55 & 0.03 \\
\hline 291 & UGC7690 & 98 & 76 & 36 & 14.68 & 0.05 & 14.31 & 0.03 \\
\hline 292 & UGC7699 & 135 & 52 & 32 & 14.74 & 0.05 & 14.45 & 0.03 \\
\hline 293 & UGC7698 & 140 & 94 & 9 & 14.88 & 0.05 & 14.71 & 0.03 \\
\hline 294 & UGC7719 & 79 & 43 & 347 & 15.99 & 0.05 & 15.79 & 0.03 \\
\hline 296 & UGC7774 & 100 & 28 & 100 & 16.34 & 0.05 & 15.77 & 0.03 \\
\hline 298 & UGCA292 & 58 & 38 & 0 & 16.46 & 0.06 & 16.36 & 0.04 \\
\hline 299 & M104 & 278 & 116 & 90 & 12.98 & 0.05 & 12.57 & 0.03 \\
\hline 300 & NGC4605 & 249 & 165 & 303 & 14.67 & 0.06 & 13.55 & 0.04 \\
\hline 301 & NGC4618 & 168 & 134 & 22 & 12.83 & 0.05 & 12.56 & 0.03 \\
\hline 302 & NGC4625 & 150 & 107 & 280 & 14.63 & 0.05 & 14.32 & 0.03 \\
\hline 303 & NGC4631 & 476 & 270 & 80 & 11.29 & 0.05 & 11.07 & 0.03 \\
\hline 304 & UGC7866 & 98 & 86 & 357 & 14.68 & 0.05 & 14.61 & 0.03 \\
\hline 305 & NGC4656 & 360 & 128 & 220 & 11.58 & 0.05 & 11.58 & 0.03 \\
\hline 306 & UGC7916 & 76 & 50 & 170 & 15.86 & 0.05 & 15.79 & 0.03 \\
\hline 309 & UGC7950 & 72 & 62 & 0 & 15.23 & 0.05 & 15.02 & 0.03 \\
\hline 310 & UGC7949 & 82 & 50 & 24 & 16.06 & 0.05 & 15.96 & 0.03 \\
\hline 311 & NGC4707 & 104 & 88 & 20 & 15.09 & 0.05 & 14.95 & 0.03 \\
\hline 312 & NGC4736 & 516 & 412 & 100 & 11.76 & 0.05 & 11.39 & 0.03 \\
\hline 313 & UGC8024 & 100 & 63 & 213 & 14.94 & 0.05 & 14.97 & 0.03 \\
\hline 315 & NGC4826 & 362 & 224 & 113 & 13.51 & 0.08 & 12.55 & 0.03 \\
\hline 316 & UGC 8091 & 62 & 46 & 32 & 15.23 & 0.06 & 15.18 & 0.03 \\
\hline 318 & UGCA319 & 65 & 45 & 24 & 16.58 & 0.06 & 16.09 & 0.03 \\
\hline 319 & UGCA320 & 246 & 82 & 114 & 13.93 & 0.08 & 13.71 & 0.04 \\
\hline 321 & UGC8188 & 190 & 163 & 90 & 13.38 & 0.05 & 13.19 & 0.03 \\
\hline 322 & UGC8201 & 134 & 75 & 90 & 14.53 & 0.05 & 14.20 & 0.03 \\
\hline 323 & MCG-03-34-002 & 65 & 40 & 320 & 16.09 & 0.05 & 15.73 & 0.03 \\
\hline 325 & UGC 8245 & 96 & 50 & 70 & 16.47 & 0.05 & 15.99 & 0.03 \\
\hline 326 & NGC5023 & 205 & 64 & 26 & 14.65 & 0.05 & 14.25 & 0.03 \\
\hline 327 & CGCG217-018 & 57 & 44 & 35 & 16.58 & 0.05 & 16.12 & 0.03 \\
\hline 329 & UGC8313 & 96 & 57 & 30 & 16.31 & 0.05 & 15.99 & 0.03 \\
\hline 330 & UGC8320 & 146 & 99 & 341 & 14.55 & 0.05 & 14.21 & 0.04 \\
\hline 331 & UGC8331 & 118 & 58 & 323 & 15.95 & 0.05 & 15.73 & 0.04 \\
\hline 332 & NGC5055 & 549 & 356 & 80 & 12.35 & 0.05 & 11.81 & 0.03 \\
\hline 333 & NGC5068 & 306 & 296 & 90 & 11.94 & 0.09 & 11.55 & 0.04 \\
\hline 336 & IC4247 & 64 & 39 & 333 & 15.97 & 0.05 & 15.55 & 0.03 \\
\hline 338 & NGC5204 & 169 & 106 & 351 & 12.95 & 0.05 & 12.72 & 0.03 \\
\hline
\end{tabular}


Table 3

(Continued)

\begin{tabular}{|c|c|c|c|c|c|c|c|c|}
\hline \multirow{2}{*}{$\begin{array}{l}\text { No. } \\
\text { (1) } \\
\end{array}$} & \multirow{2}{*}{$\begin{array}{c}\text { Galaxy Name } \\
\text { (2) }\end{array}$} & \multirow{2}{*}{$\begin{array}{l}a \\
(") \\
(3) \\
\end{array}$} & \multirow{2}{*}{$\begin{array}{l}b \\
(") \\
(4)\end{array}$} & \multirow{2}{*}{$\begin{array}{l}\text { P.A. } \\
\text { (deg) } \\
(5)\end{array}$} & \multicolumn{2}{|c|}{$\begin{array}{l}\text { FUVV }_{\text {LocalVolumeLegacy }} \\
\text { (mag) }\end{array}$} & \multicolumn{2}{|c|}{$\begin{array}{l}\text { NUV }_{\text {LocalVolumeLegacy }} \\
\text { (mag) }\end{array}$} \\
\hline & & & & & (6) & (7) & $(8)$ & (9) \\
\hline 339 & NGC5194 & 850 & 565 & 15 & 11.01 & 0.05 & 10.53 & 0.03 \\
\hline 340 & NGC5195 & 102 & 96 & 0 & 15.72 & 0.06 & 14.65 & 0.03 \\
\hline 341 & UGC8508 & 80 & 60 & 305 & $\ldots$ & $\ldots$ & $\ldots$ & $\ldots$ \\
\hline 344 & NGC5229 & 140 & 51 & 347 & 15.70 & 0.07 & 15.35 & 0.03 \\
\hline 345 & NGC5238 & 108 & 83 & 0 & 15.19 & 0.05 & 14.98 & 0.03 \\
\hline 347 & [KK98]208 & 180 & 75 & 57 & $<23.75$ & & $<23.90$ & \\
\hline 348 & NGC5236 & 550 & 528 & 0 & 10.07 & 0.05 & 9.59 & 0.03 \\
\hline 349 & ESO444-G084 & 63 & 44 & 310 & 16.05 & 0.05 & 15.82 & 0.03 \\
\hline 350 & UGC8638 & 90 & 66 & 73 & 15.76 & 0.05 & 15.57 & 0.03 \\
\hline 351 & UGC8651 & 97 & 70 & 59 & 15.63 & 0.05 & 15.52 & 0.03 \\
\hline 352 & NGC5253 & 160 & 122 & 44 & 12.29 & 0.05 & 11.94 & 0.03 \\
\hline 354 & NGC5264 & 134 & 113 & 66 & 14.69 & 0.06 & 14.28 & 0.06 \\
\hline 359 & UGC8760 & 108 & 56 & 29 & 15.84 & 0.05 & 15.61 & 0.03 \\
\hline 360 & UGC8837 & 182 & 74 & 17 & $\ldots$ & $\ldots$ & $\ldots$ & $\ldots$ \\
\hline 361 & UGC8833 & 60 & 58 & 0 & 16.64 & 0.05 & 16.42 & 0.04 \\
\hline 363 & NGC5457 & 900 & 723 & 37 & 9.98 & 0.05 & 9.83 & 0.03 \\
\hline 365 & NGC5474 & 206 & 186 & 90 & 12.94 & 0.05 & 12.84 & 0.03 \\
\hline 366 & NGC5477 & 84 & 62 & 64 & 14.89 & 0.05 & 14.90 & 0.03 \\
\hline 367 & KKR03 & 34 & 24 & 0 & 18.43 & 0.06 & 18.22 & 0.03 \\
\hline 370 & UGC9128 & 64 & 44 & 36 & 16.30 & 0.07 & 15.90 & 0.04 \\
\hline 372 & NGC5585 & 196 & 133 & 38 & 13.14 & 0.05 & 12.81 & 0.03 \\
\hline 375 & UGC9240 & 111 & 91 & 90 & 14.82 & 0.05 & 14.57 & 0.03 \\
\hline 378 & UGC9405 & 100 & 68 & 333 & 16.70 & 0.07 & 16.31 & 0.03 \\
\hline 379 & MRK475 & 36 & 36 & 196 & 17.37 & 0.05 & 17.13 & 0.03 \\
\hline 382 & NGC5832 & 146 & 90 & 49 & 14.58 & 0.05 & 14.29 & 0.03 \\
\hline 386 & NGC5949 & 110 & 68 & 324 & 15.15 & 0.05 & 14.72 & 0.03 \\
\hline 388 & UGC9992 & 78 & 54 & 340 & 16.33 & 0.06 & 16.04 & 0.03 \\
\hline 395 & NGC6503 & 320 & 116 & 119 & 13.22 & 0.05 & 12.78 & 0.03 \\
\hline 406 & IC4951 & 112 & 46 & 355 & 15.08 & 0.05 & 14.95 & 0.03 \\
\hline 412 & DDO210 & 80 & 38 & 103 & 16.65 & 0.05 & 16.35 & 0.04 \\
\hline 415 & IC5052 & 225 & 84 & 323 & 13.90 & 0.05 & 13.36 & 0.03 \\
\hline 418 & NGC7064 & 125 & 40 & 90 & 14.17 & 0.05 & 13.90 & 0.03 \\
\hline 419 & NGC7090 & 270 & 80 & 308 & 14.10 & 0.05 & 13.53 & 0.04 \\
\hline 420 & IC5152 & 156 & 137 & 90 & 12.44 & 0.05 & 12.02 & 0.03 \\
\hline 423 & IC5256 & 62 & 38 & 22 & 16.72 & 0.05 & 16.32 & 0.03 \\
\hline 426 & UGCA438 & 94 & 82 & 0 & 15.30 & 0.05 & 15.24 & 0.03 \\
\hline 427 & ESO347-G017 & 106 & 64 & 90 & 15.59 & 0.05 & 15.31 & 0.03 \\
\hline 428 & UGC12613 & 230 & 134 & 113 & 15.68 & 0.06 & 14.90 & 0.06 \\
\hline 430 & IC5332 & 322 & 286 & 0 & 12.58 & 0.05 & 12.37 & 0.03 \\
\hline 431 & NGC7713 & 185 & 114 & 345 & $\ldots$ & $\ldots$ & $\ldots$ & $\ldots$ \\
\hline 433 & UGCA442 & 122 & 58 & 43 & 14.86 & 0.05 & 14.70 & 0.03 \\
\hline 435 & ESO149-G003 & 124 & 50 & 332 & 15.63 & 0.05 & 15.57 & 0.03 \\
\hline 436 & NGC7793 & 378 & 250 & 90 & 11.15 & 0.05 & 10.98 & 0.03 \\
\hline 1 & ESO410-G005 & 61 & 45 & 308 & 18.32 & 0.08 & 17.20 & 0.04 \\
\hline 2 & Sculptor-dE1 & 80 & 52 & 0 & (23.60) & $\ldots$ & 18.92 & 0.17 \\
\hline 3 & ESO294-G010 & 82 & 51 & 0 & 18.08 & 0.08 & 17.54 & 0.04 \\
\hline 4 & ESO540-G030 & 84 & 74 & 0 & 19.19 & 0.10 & 18.13 & 0.08 \\
\hline 5 & ESO540-G032 & 50 & 46 & 0 & 19.53 & 0.10 & 18.66 & 0.06 \\
\hline 6 & UGC00521 & 54 & 54 & 90 & 16.02 & 0.05 & 16.04 & 0.03 \\
\hline 7 & NGC404 & 105 & 105 & 90 & 16.18 & 0.10 & 14.45 & 0.04 \\
\hline 8 & IC2049 & 60 & 53 & 0 & 16.46 & 0.05 & 16.12 & 0.03 \\
\hline 9 & UGCA133 & 108 & 76 & 0 & (23.07) & $\ldots$ & (23.07) & $\ldots$ \\
\hline 10 & F8D1 & 127 & 127 & 90 & 18.11 & 2.30 & 17.27 & 0.43 \\
\hline 11 & [FM2000]1 & 44 & 44 & 90 & (23.95) & $\ldots$ & 19.98 & 0.06 \\
\hline 12 & LEDA166101 & 110 & 76 & 33 & (22.24) & $\ldots$ & 17.98 & 0.10 \\
\hline 13 & Arp'sLoop & 68 & 68 & 90 & 18.63 & 0.09 & 18.14 & 0.05 \\
\hline 14 & KKH057 & 36 & 26 & 45 & (23.24) & $\ldots$ & 20.75 & 0.18 \\
\hline 15 & AM1001-270 & 84 & 48 & 319 & 17.46 & 0.21 & 16.81 & 0.09 \\
\hline 16 & $\mathrm{BK} 05 \mathrm{~N}$ & 104 & 51 & 330 & (23.18) & $\ldots$ & 20.26 & 1.20 \\
\hline 17 & UGC05442 & 98 & 62 & 34 & $(23.42)$ & $\ldots$ & 18.16 & 0.05 \\
\hline 18 & IKN & 90 & 78 & 180 & (22.90) & $\ldots$ & (23.06) & $\ldots$ \\
\hline 19 & [HS98]117 & 106 & 64 & 0 & $(20.82)$ & $\ldots$ & (20.98) & $\ldots$ \\
\hline 20 & DDO078 & 70 & 70 & 90 & $(22.01)$ & $\ldots$ & (22.05) & $\ldots$ \\
\hline
\end{tabular}


Table 3

(Continued)

\begin{tabular}{|c|c|c|c|c|c|c|c|c|}
\hline \multirow{2}{*}{$\begin{array}{l}\text { No. } \\
\text { (1) }\end{array}$} & \multirow{2}{*}{$\begin{array}{c}\text { Galaxy Name } \\
\text { (2) }\end{array}$} & \multirow{2}{*}{$\begin{array}{c}a \\
\left({ }^{\prime \prime}\right) \\
(3)\end{array}$} & \multirow{2}{*}{$\begin{array}{l}b \\
\left({ }^{\prime \prime}\right) \\
(4)\end{array}$} & \multirow{2}{*}{$\begin{array}{l}\text { P.A. } \\
\text { (deg) } \\
(5)\end{array}$} & \multicolumn{2}{|c|}{$\begin{array}{c}\text { FUV }_{\text {LocalVolumeLegacy }} \\
\text { (mag) }\end{array}$} & \multicolumn{2}{|c|}{$\begin{array}{c}\mathrm{NUV}_{\text {LocalVolumeLegacy }} \\
\text { (mag) }\end{array}$} \\
\hline & & & & & (6) & (7) & (8) & (9) \\
\hline 21 & BK06N & 116 & 54 & 304 & $(23.39)$ & $\ldots$ & $(23.30)$ & $\ldots$ \\
\hline 22 & UGC06782 & 68 & 57 & 115 & 17.14 & 0.06 & 16.75 & 0.04 \\
\hline 23 & ESO321-G014 & 86 & 52 & 22 & 16.44 & 0.14 & 16.14 & 0.04 \\
\hline 24 & UGC07321 & 185 & 39 & 81 & 16.05 & 0.05 & 15.40 & 0.03 \\
\hline 25 & ККH086 & 66 & 42 & 0 & 19.30 & 0.09 & 18.58 & 0.06 \\
\hline 26 & KKR25 & 47 & 44 & 0 & $(23.45)$ & $\ldots$ & 19.09 & 0.06 \\
\hline 27 & ККH098 & 63 & 40 & 5 & 17.18 & 0.06 & 16.94 & 0.04 \\
\hline
\end{tabular}

(This table is also available in a machine-readable form in the online journal.)

in the zero point, as well as the uncertainty due to the errorweighted fit of the growth curve.

In Table 3, we list only those galaxies in the Local Volume Legacy sample, providing the following.

Column 1: index number, as in Table 1.

Column 2: galaxy name, as in Table 1.

Columns 3-5: semimajor axis, semiminor axis, and position angle of the elliptical annulus used by Dale et al. (2009) to extract photometry from Spitzer IRAC and MIPS imaging, reproduced from Table 1 of Dale et al. (2009).

Columns 6-9: FUV and NUV magnitude and error, measured in the common elliptical aperture described by the preceding columns. Corrections for MW foreground extinction have been applied using the $E(B-V)$ values listed here, and the Cardelli et al. (1989) extinction law with $R_{V}=3.1$. That is, $A_{\mathrm{FUV}}=$ $7.9 E(B-V)$ and $A_{\mathrm{FUV}}=8.0 E(B-V)$. For non-detections, $3 \sigma$ point source upper limits are listed, enclosed in parenthesis. The quoted errors include the photometric uncertainty within the aperture and the error in the zero point $(0.05$ and 0.03 $m_{\mathrm{AB}}$ in the FUV and NUV, respectively; Morrissey et al. 2007).

\section{RESULTS AND DISCUSSION}

\subsection{Basic Sample Properties}

The GALEX observations presented here significantly improve the depth to which complete, statistical sampling of Local Volume star-forming (aka, "blue sequence") galaxies is achieved. This is illustrated in Figure 2, which shows the number distributions of FUV and NUV absolute magnitudes. The filled histograms represent GALEX's resultant coverage of the primary $11 \mathrm{HUGS}$ target volume $\left(|b|<30^{\circ}, d<11\right.$ Mpc) prior to our observations, and the solid line shows the distributions when our observations are included. While the more limited sample with prior coverage begins to become incomplete for $\gtrsim-15$ mag, our sample continues to increase until about -13 mag. 11HUGS mitigates the previous $\sim 4$-fold underrepresentation of Local Volume galaxies at low luminosities.

Luminosity functions based on surveys that have welldetermined selection functions and cover much larger volumes than 11HUGS also provide an interesting point of comparison for our $M_{\mathrm{FUV}}$ and $M_{\mathrm{NUV}}$ number distributions. Such luminosity functions are subject to typical biases toward the most luminous, massive, high-surface brightness systems, and therefore are generally well sampled at the bright end, but are not robustly constrained in the dwarf galaxy regime. In Figure 2, we over- plot UV luminosity functions, ${ }^{18}$ as computed by Schiminovich et al. (2007), for disk galaxies in the SDSS DR4 spectroscopic sample (Adelman-McCarthy et al. 2006) that have also been observed in the GALEX Medium Imaging Survey (Martin et al. 2005). The curves give their best fitting Schechter function fits, with the dashed segments representing extrapolations past the last data point shown. To force approximate agreement between the number distributions and the Schiminovich et al. (2007) luminosity functions at $\sim L^{*}$, the latter has been multiplied by two times the $|b|>30, d<11$ Mpc 11HUGS target volume. The factor of two implies that the portion of the Local Volume probed by 11 HUGS is over-dense compared with cosmological volumes, as discussed in Karachentsev et al. (2004) and Lee et al. (2009a). Qualitatively, there is good agreement between the relative shapes of both $M_{\mathrm{FUV}}$ and $M_{\mathrm{NUV}}$ luminosity functions and the 11HUGS number distributions prior to the sharp falloff of galaxies fainter than $\sim-13$ mag. This provides some assurance that the 11HUGS sampling of bright galaxies, while sparse due to the small target volume, is not heavily biased relative to the global distribution of UV luminosities. The extrapolated faint-end slopes of the luminosity functions appear to also be reasonable in comparison with our local data set. In $M_{\mathrm{FUV}}$, the $11 \mathrm{HUGS}$ data set appears to prefer a slightly steeper rise at the faint end ( $\alpha \sim 1.06$; gray), though this is well within the uncertainties of the value of $\alpha=1.02 \pm 0.05$ reported by Schiminovich et al. (2007). If the standard SFR conversion recipe of Kennicutt (1998b) is applied to the FUV luminosities, the SFRs probed by the sample span roughly six orders of magnitude, from $\sim 10 M_{\odot} \mathrm{yr}^{-1}$ to $\sim 10^{-6} M_{\odot} \mathrm{yr}^{-1}$.

The dominance of low-luminosity star-forming galaxies in the sample is also illustrated by distributions in the FUV - NUV color (corrected for MW reddening, but not for dust attenuation internal to the galaxies themselves). In Figure 3, we show a histogram of the UV color and also plot it as a function of $\mathrm{EW}(\mathrm{H} \alpha), M_{\mathrm{FUV}}$, and $M_{B}$. The points are coded to indicate different morphological types, and the histogram is shaded in a corresponding manner. The sample is concentrated in a fairly narrow range of UV color: the median is $0.29 \mathrm{mag}$, and $79 \%$ of the galaxies have $0<$ FUV - NUV $<0.5$. Such blue colors are primarily a result of the recent star formation activity in such systems (e.g., van Zee 2001; Hunter \& Elmegreen 2004; Lee et al. 2007), while low metallicities (e.g., Skillman et al. 1989; van Zee et al. 1997; Lee et al. 2004) and low dust attenuation (e.g., Lee et al. 2009b) also play a role.

\footnotetext{
18 The luminosity functions were rescaled to reflect $H_{0}=75 \mathrm{~km} \mathrm{~s}^{-1} \mathrm{Mpc}^{-1}$ as is assumed here to calculate distances for objects without standard candle/ruler estimates.
} 

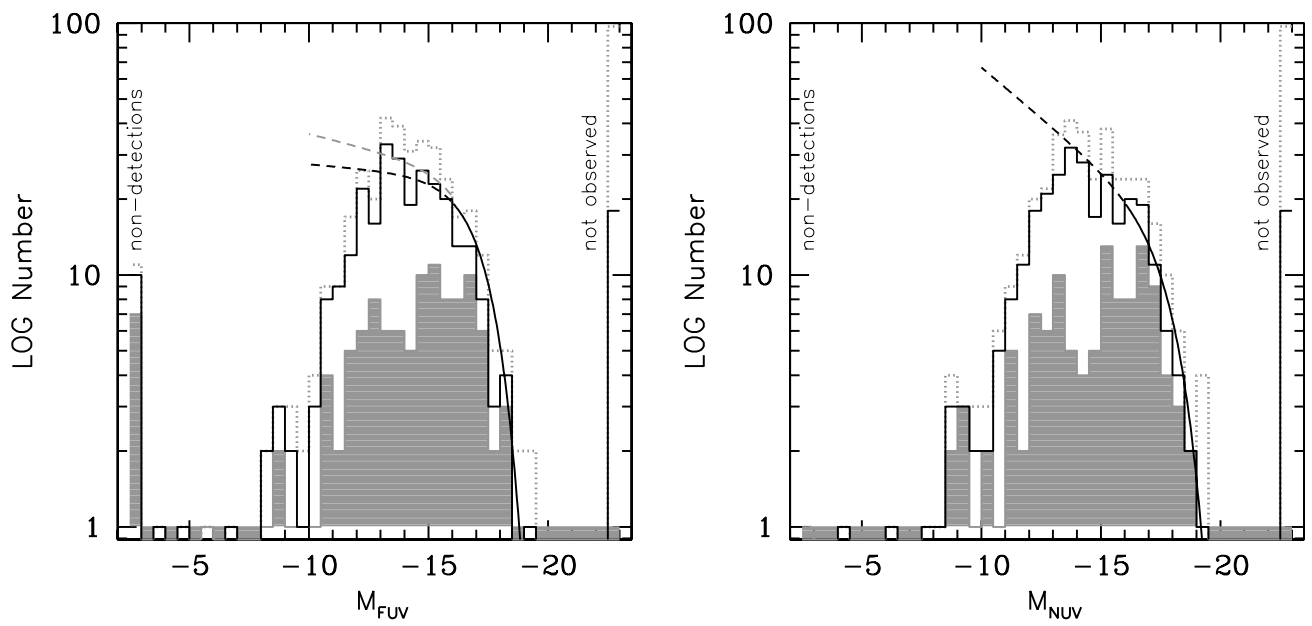

Figure 2. Number distributions in NUV and FUV absolute magnitude for all galaxies with GALEX data in the overall parent (Paper I) sample (dotted gray histogram), and the subset of the sample which is most complete: spiral and irregular galaxies $(T>0)$ which avoid the plane of the MW $|b|>30^{\circ}$ (black histogram). Corrections for foreground MW extinction, but not extinction internal to the galaxies, have been applied. The gray area shows the GALEX coverage of the $|b|>30^{\circ}, T>0$ galaxies prior to observations by 11HUGS. Non-detections are plotted at the far left of the figure, while the number of galaxies that have not been observed by GALEX are shown at the far right. For comparison, we overplot Schechter fits to GALEX luminosity functions computed by Schiminovich et al. (2007) for disk-dominated galaxies in the Sloan Digital Sky Survey (SDSS) spectroscopic sample (solid curve; the dashed portion is an extrapolation from the last data point shown). These luminosity functions have been multiplied by twice the $|b|>30, d<11 \mathrm{Mpc} 11 \mathrm{HUGS}$ survey volume to provide approximate agreement with the observed number distributions (see Section 3.1 for the discussion). Our GALEX program significantly improves the depth to which complete, statistical sampling of Local Volume star-forming galaxies is achieved.
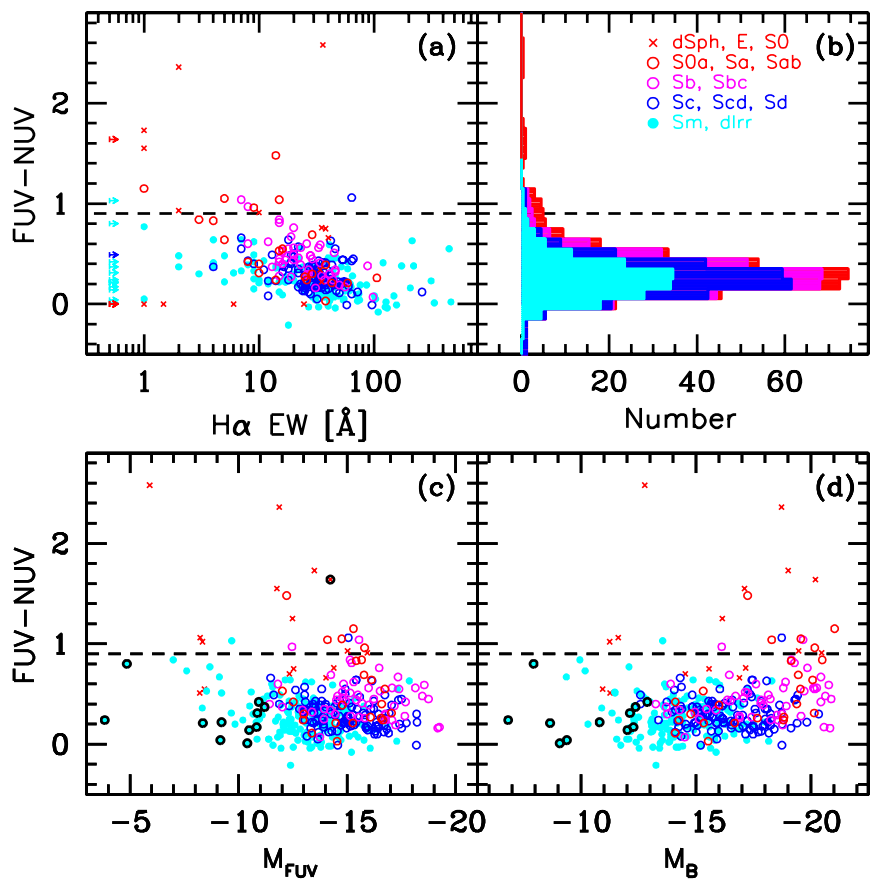

Figure 3. Distributions of the integrated FUV - NUV color. Corrections for MW reddening, but not dust attenuation internal to the galaxies, have been applied. Different symbols and colors are used to distinguish between morphological type as indicated in panel (b). Dashed lines are drawn at FUV - NUV $=0.9$, which Gil de Paz et al. (2007) have determined to provide good separation between early-type elliptical and lenticular "red sequence" galaxies, and late-type spiral and irregular "blue sequence" galaxies. Galaxies which were undetected in $\mathrm{H} \alpha$ narrowband imaging, but have one-orbit-depth GALEX FUV imaging are circled in black, and largely exhibit blue UV colors characteristic of star-forming galaxies. Blue star-forming dwarf galaxies dominate the sample by construction.

(A color version of this figure is available in the online journal.)

Late-type spiral and irregular "blue sequence" galaxies can be roughly separated from early-type "red sequence" galaxies using a division at FUV - NUV $=0.9$ (plotted as a dashed line in Figure 3) as described in Gil de Paz et al. (2007). Again, by design our sample does not contain many early-type galaxies, and there are few galaxies with FUV - NUV $>0.9$. For the star-forming galaxies with FUV - NUV $<0.9$, the UV color does not show strong trends with either $M_{\mathrm{FUV}}$ or $M_{B}$, except perhaps for slightly redder values at $M_{B}$ brighter than -19 , at which the sample is predominantly composed of earlier type spirals. There is also a weak trend toward redder colors at lower equivalent width. This trend is expected, since $\operatorname{EW}(\mathrm{H} \alpha)$ traces the current SFR relative to the past lifetime average, which provides a measure of the recent star formation history.

\subsection{GALEX Non-detections}

Late-type galaxies in the Local Volume that do not have measurable UV emission appear to very rare, at least among the $B<15.5$ population where our observations are concentrated (see Figure 1). Only 22 of the 390 galaxies in our sample which were observed by GALEX were not detected in the FUV. About half of these $(N=12)$ are galaxies classified as faint dwarf spheroidals/ellipticals and would not be expected to have UV emission from the recent star formation. ${ }^{19}$ Another eight are classified as late-type dwarfs, but only have shallow imaging $(\lesssim 200$ s) available; in these cases a detection is also not expected given the galaxies' low $B$-band luminosities and/or low $\mathrm{H} \alpha$-based SFRs. ${ }^{20}$ The remaining two, UGC5428 and UGCA276, do have deep GALEX imaging $(t \sim 1700 \mathrm{~s})$ and are cases for which further follow-up may be valuable as described further below. Both have extremely low luminosities with $M_{B} \sim-12$ and are nearby $(D \sim 3 \mathrm{Mpc})$. The GALEX imaging of these galaxies is sensitive enough to detect a single early-type B star $\left(\sim 15 M_{\odot}\right)$.

UGC5428 is a member of the M81 group and has been classified as a "transition" dwarf-a system whose properties are

\footnotetext{
19 Sculptor-dE1, UGCA133, FM2000-1, LEDA166101, KKH057, BK05N, UGC5442, IKN, DDO78, BK06N, [KK98]208, and KKR25.

20 IC2782, LSBCD565-06, LSBCD634-03, UGCA86, KKH34, UGCA92, HS[98]117, and LEDA166115.
} 
intermediate between those of late- and early-type galaxies (e.g., Mateo 1998; Grebel et al. 2003). Although it is seen to be dominated by an old stellar population based upon ANGST HST imaging (D. R. Weisz et al. 2011, in preparation), and H I gas has not been observed to a limit of $\sim 10^{6} M_{\odot}$ (Karachentsev \& Kaisin 2007), a few faint, compact $\mathrm{H} \alpha$ knots have been tentatively identified in narrowband imaging (Karachentsev \& Kaisin 2007; Paper I). HST stellar color-magnitude diagrams have been used to reconstruct the star formation history, indicating that the average SFR over the past Gyr is $\sim 8 \times 10^{-4} M_{\odot} \mathrm{yr}^{-1}$, but with error bars consistent with no star formation (D. R. Weisz et al. 2011, in preparation). The narrowband excess sources may therefore be background galaxies. Follow-up spectroscopy is required to confirm the nature of these detections. No significant FUV emission is measurable in apertures spanning the extent of the galaxy, but photometry with a smaller aperture limited to the region where the narrowband excess sources are found, yields an FUV flux that is consistent with that expected from the $\mathrm{H} \alpha$ emission within large uncertainties $\left(m_{\mathrm{FUV}}=24.5 \pm 3.3\right)$. The faint, extended diffuse emission observed in the NUV likely arises from older main-sequence turn-off stars.

UGCA276 belongs to a prominent association of dwarf galaxies around NGC 4214 at 2.9 Mpc (Tully et al. 2006). Morphologically, it has been classified as a dwarf irregular. There is a published HI flux for the system, though the detection is uncertain (de Vaucouleurs et al. 1991; Karachentsev et al. 2004). However, there is no observable $\mathrm{H} \alpha$ emission (Paper I). The NUV emission in this system is also faint, extended, and diffuse, so it is not likely to arise from very recent star formation. HST resolved stellar population imaging from ANGST also shows that UGCA276 is dominated by an old stellar population and has had little recent activity. Similar to UGC5428, the reconstructed star formation history over the past Gyr is $\sim 4 \times 10^{-4} M_{\odot} \mathrm{yr}^{-1}$, but with error bars consistent with zero (D. R. Weisz et al. 2011, in preparation). If the detection of $\mathrm{H} \mathrm{I}$ is found to be robust, this would make the system extremely peculiar as UGCA276 would be the only galaxy in our sample with detectable $\mathrm{H}$ I gas but no evidence of recent star formation. Further detailed study of both objects would be useful to establish the basis of their current evolutionary status and to understand the possible impact of their group environments.

In the NUV, only 10 galaxies were not detected and are a subset of the FUV non-detections. Half are dwarf irregulars which only have shallow exposures $(\lesssim 200 \mathrm{~s}),{ }^{21}$ and the other half are dwarf spheroidals. ${ }^{22}$

The nearly ubiquitous presence of UV emission in local spirals and irregulars is not surprising given that they are starforming systems, and moreover, because $\mathrm{H} \alpha$ has already been detected in the vast majority of late-type galaxies surveyed (e.g., Meurer et al. 2006; James et al. 2008; Paper I). This finding reinforces the idea that the intermittent cessation of activity is uncommon, as we rarely observe such systems in an "off" mode, at least for the $B<15.5$ population probed (Lee et al. 2009a). The result is particularly relevant for dwarf galaxies, which generally appear to have fluctuating or bursty star formation histories (e.g., Weisz et al. 2008; Tolstoy et al. 2009, and references therein), because it implies that the fluctuations do not go to zero for durations comparable to the lifetimes of UV emitting stars $(\sim 100 \mathrm{Myr})$. For the higher luminosity galaxies

\footnotetext{
${ }^{21}$ HS98_117, UGCA86, UGCA92, KKH34, and LSBCD634-03.

22 UGCA133, IKN, DDO078, BK06N, and [KK98]208.
}

in the sample $\left(M_{B} \lesssim-14\right)$, star formation over galaxy-wide scales further appears to be virtually continuous-the $100 \% \mathrm{H} \alpha$ detection rate for this subset of our sample (Paper I; Lee et al. 2009a) constrains gaps in activity to be less than the lifetimes of ionizing stars $(\lesssim 10 \mathrm{Myr})$.

It is not clear whether this picture continues to be true for starforming dwarfs with lower luminosities. In Lee et al. (2009a), it was noted that all of the late-type galaxies which were undetected in the Paper I H $\alpha$ survey had very low luminosities, with $-7.6 \leqslant M_{B} \leqslant-13.6$. Thus, it is possible that off-modes may be a general feature of the star formation histories of this extreme population. However, it is not straightforward to interpret the lack of $\mathrm{H} \alpha$ as an absence of star formation. Such systems have lifetime-averaged SFRs on the order of $10^{-3}-10^{-4} M_{\odot} \mathrm{yr}^{-1}$, so Poisson fluctuations in the sampling of the IMF may lead to the absence of $O$-stars, even when continuous star formation is in fact occurring. The FUV should be more immune to this Poisson noise, and as we discuss below, sufficiently deep GALEX observations of $\mathrm{H} \alpha$ non-detections show them to generally have normal levels of recent star formation activity relative to the broader population of dwarf irregular galaxies. We may therefore speculate that extremely low-luminosity dwarfs also do not commonly undergo intermittent periods of complete inactivity which last for $\gtrsim 100 \mathrm{Myr}$. This is only a speculation because our sample is incomplete in this luminosity regime. Obtaining $\mathrm{H} \alpha$ and FUV observations of a large complete sample of extremely low-luminosity galaxies, or better yet, of a complete sample of galaxies with low $\mathrm{H}_{\mathrm{I}}$ masses $\left(M_{\mathrm{HI}} \leqslant 10^{8} M_{\odot}\right)$, is required to examine whether the rarity of UV non-detections (or equivalently the rarity of temporarily halted star formation) persists.

\subsection{A Re-examination of Star Formation Properties in the FUV}

In light of the finding that GALEX FUV observations may be a more robust probe of star formation in low-density environments than typical $\mathrm{H} \alpha$ narrowband imaging (see the introduction), it is instructive to use our dwarf galaxy dominated sample to re-examine commonly studied star formation properties which have been largely based on $\mathrm{H} \alpha$ measurements over the past three decades. Here, we illustrate how our view of evolutionary status of dwarf galaxies may change by recalculating two coarse measures of the global star formation efficiency: the SFR per unit $\mathrm{HI}_{\mathrm{I}}$ mass $\left(M_{\mathrm{HI}}\right)$ and the SFR per unit stellar mass $\left(M_{*}\right)$. These quantities provide the basis for computing timescales of future and past star formation and are related to the "gas consumption timescale" and the "stellar birthrate," respectively (e.g., Kennicutt 1983; Hunter \& Gallagher 1985; Kennicutt et al. 1994; van Zee et al. 1997; van Zee 2001; Skillman et al. 2003; Brinchmann et al. 2004; Lee et al. 2004, 2009a; Karachentsev \& Kaisin 2007; James et al. 2008; Côté et al. 2009; Bothwell et al. 2009). In this context, it is also particularly interesting to examine the UV properties of late-type galaxies that were not detected in earlier $\mathrm{H} \alpha$ imaging and thus appeared to be devoid of star formation.

\subsubsection{Global Star Formation Efficiencies}

In Figure 4, the SFR is plotted against $M_{\mathrm{H} \text { I }}$ in the left panel, and SFR/ $M_{\mathrm{HI}}$ is shown as a function of $M_{B}$ in the right. There are two sets of points: the gray set gives the value of the ordinate using $\mathrm{H} \alpha$-based SFRs, while the colored set shows those based upon the FUV. Only galaxies that have both FUV and $\mathrm{H} \alpha$ measurements are shown. The SFRs are computed as in Lee 

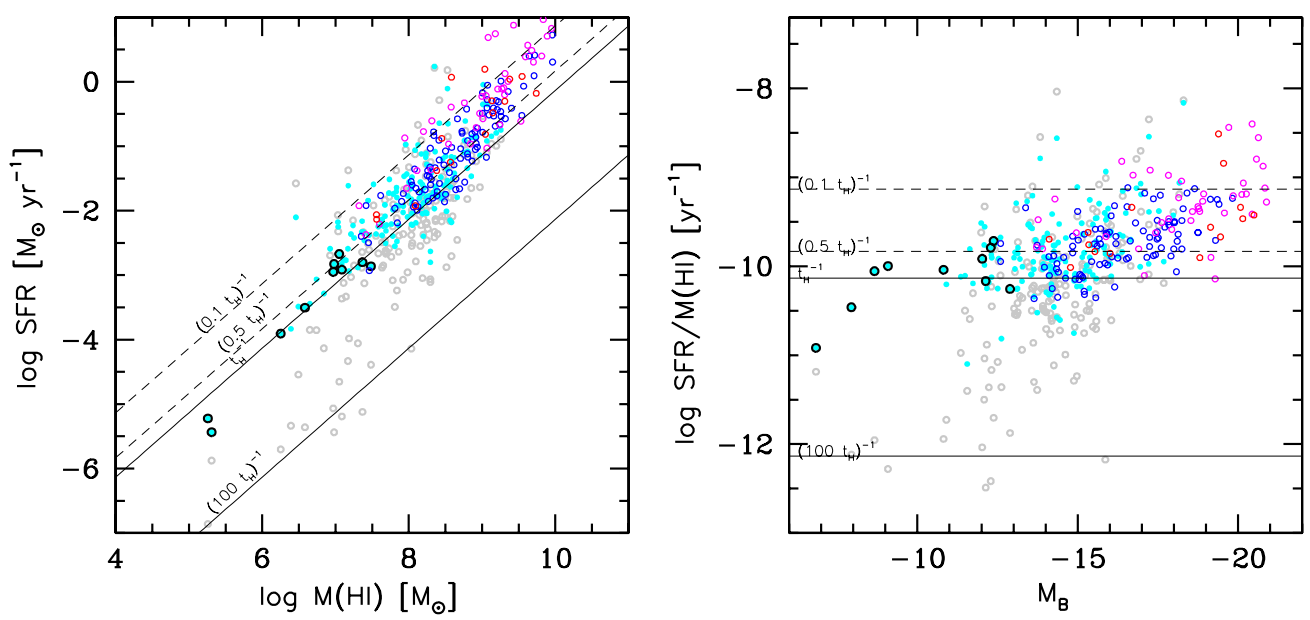

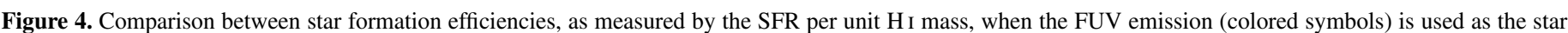

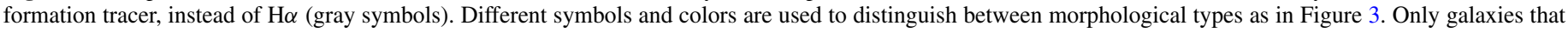
have both FUV and $\mathrm{H} \alpha$ measurements are shown. Best-effort attenuation corrections are applied before computing the SFR as in Lee et al. (2009b).

(A color version of this figure is available in the online journal.)

et al. (2009b), where best-effort corrections have been applied for internal dust attenuation. ${ }^{23}$

The H I masses are computed from single-dish $21 \mathrm{~cm}$ line fluxes compiled from the literature as described in Lee et al. (2009a), where the measurements are primarily drawn from Springob et al. (2005); the H I Parkes All Sky Survey (HIPASS) catalog as published in Meyer et al. (2004); and the homogenized H I compilation of Paturel et al. (2003) as made available through the Hyperleda database. The lines in Figure 4 indicate values of constant SFR $/ M_{\mathrm{HI}}$, in units of the age of the universe $\left(t_{\mathrm{H}}=\right.$ 13.7 Gyr).

The difference between the global star formation efficiencies inferred from the two SFR tracers is immediately apparent. The $\mathrm{H} \alpha$-based values yield significantly lower efficiencies for the late-type dwarf galaxies with $M_{B} \gtrsim-15$ and $M_{\mathrm{H}_{\mathrm{I}}} \lesssim 10^{8.5}$ relative to the more luminous and more massive spirals. This behavior is consistent with the results of many previous authors who have reported gas depletion timescales $\left(\propto M_{\mathrm{H}_{\mathrm{I}}} / \mathrm{SFR}\right)$ in excess of several Hubble times for samples of dwarf irregular galaxies (e.g., van Zee 2001; Skillman et al. 2003; Karachentsev \& Kaisin 2007; Knapen \& James 2009; Bothwell et al. 2009). In contrast, the FUV-based values generally do not fall below $t_{\mathrm{H}}^{-1}$. Most spiral and irregular galaxies in our Local Volume sample have SFR(UV)/H I values on the order of $t_{\mathrm{H}}^{-1}$, with $75 \%$ between $t_{\mathrm{H}}^{-1}$ and $0.1 t_{\mathrm{H}}^{-1}$. Analogous plots for the SFR per unit stellar mass are shown in Figure 5, where the stellar masses are computed using $M_{B}$ and mass-to-light ratios based on the $B-V$ color, as in Bothwell et al. (2009). Similar differences between the $\mathrm{H} \alpha$ - and UV-based values are evident. Therefore, dwarf irregular galaxies may not be as drastically inefficient at forming stars as previously determined.

\footnotetext{
23 Briefly, the $\mathrm{H} \alpha$ attenuation is measured from the Balmer decrement, for galaxies with available integrated optical spectroscopy. The FUV attenuation is measured from the total infrared-to-UV flux ratio, for galaxies with available Spitzer IR imaging. Otherwise, empirical scaling relations are used to estimate the attenuation from $M_{B}$. There is some current debate on whether the discrepancies between the UV and $\mathrm{H} \alpha$ SFRs may be an artifact of uncertain attenuation corrections (e.g., Boselli et al. 2009; Meurer 2010). However, it is important to note that in the present sample of dwarf galaxies, there is a trend of decreasing $\mathrm{H} \alpha$-to-FUV flux prior to any attenuation corrections. Thus, the conclusions reported both here and in Lee et al. (2009b) should be robust to the effects of dust.
}

Of course, a proper analysis of star formation efficiencies requires accounting for a number of other factors that we have not considered here, for example, the molecular gas mass and the mass returned to the interstellar medium through stellar evolutionary processes (e.g., Kennicutt et al. 1994; Bigiel et al. 2008). However, this exercise is not meant to be a detailed examination of efficiencies, as would be required, for example, in a study of the Schmidt Law (Schmidt 1959; Kennicutt 1998a). Rather, the purpose is to provide a cautionary illustration of the significant differences that can arise in our understanding of the star formation properties of galaxies in the low-density regime, when the FUV is used to trace the SFR instead of $\mathrm{H} \alpha$. It is important to carefully re-evaluate previous conclusions regarding the evolutionary state of such galaxies that have been based on $\mathrm{H} \alpha$ measurements.

In this context of re-examining previous $\mathrm{H} \alpha$-based results, we also note that another similar analysis of star formation efficiencies (discussed in terms of the gas depletion and stellar mass buildup times) was recently presented by Pflamm-Altenburg et al. (2009), but under the premise that the true SFR can be recovered from $\mathrm{H} \alpha$ measurements given their non-universal model of the stellar IMF. Briefly, in the "integrated galactic stellar initial mass function" (IGIMF; Kroupa \& Weidner 2003; Weidner \& Kroupa 2005, 2006), it is assumed that (1) all stars form in clusters; (2) the maximum mass of a star formed in a cluster is a deterministic function of the cluster mass, and this maximum mass is lower than would be expected from random sampling of a universal IMF; and (3) the most massive cluster that is formed in a galaxy is dependent on its SFR. Thus, according to the IGIMF model, the stellar mass distribution of galaxies with low SFRs will be deficient in massive stars (and more deficient than would be expected from simple Poisson sampling), leading to a nonlinear relationship between the SFR and the $\mathrm{H} \alpha$ luminosity. The results given by Figures 4 and 5 are qualitatively consistent with those of Pflamm-Altenburg et al. (2009), in that the majority of galaxies have efficiencies greater than $t_{\mathrm{H}}^{-1}$ when the UV flux is used to measure the star formation activity. However, in our analysis, no change in the assumptions about the form of $I M F$ was required to arrive at this conclusion. Instead, we make the more conservative assumption that the FUV luminosity provides a more robust measure of the recent SFR than $\mathrm{H} \alpha$ in the low-density regime because it (1) primarily originates from a 

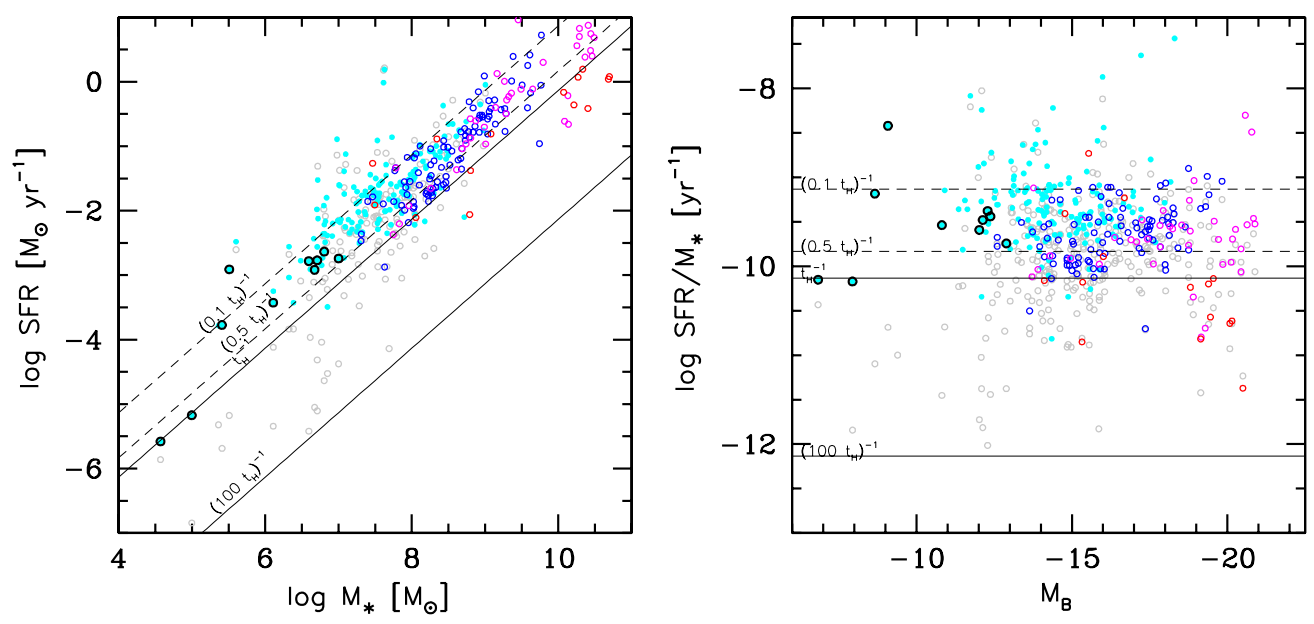

Figure 5. Same as Figure 4, but for the SFR per unit stellar mass.

(A color version of this figure is available in the online journal.)

more abundant population of B-stars and thus is not as prone to stochastic effects and (2) is emitted directly from the stellar photospheres and so does not suffer from possible uncertainties in photoionization of gas in low-density media. We do not yet draw a firm conclusion on the underlying cause of the $\mathrm{H} \alpha / \mathrm{UV}$ systematic, as this requires further investigation as discussed in Lee et al. (2009b), and references therein, and in Eldridge (2010).

Finally, it should be noted that the optical limit of the current sample may select against star-forming dwarf galaxies with low UV-based efficiencies $\left(<t_{\mathrm{H}}^{-1}\right)$. The $B=15.5$ limit corresponds to $\log (\mathrm{SFR})=-2.6$, for a typical FUV-B color of 2.5 (Gil de Paz et al. 2007) at a distance of $8.5 \mathrm{Mpc}$, which encloses approximately half the volume of our survey. Therefore, the apparent bound of the UV-based efficiencies shown in Figures 4 and 5 to values greater than $t_{\mathrm{H}}^{-1}$ should be tested using a sample with a fainter optical limit or more ideally with an $\mathrm{H}$ I-selected sample complete to masses of about $10^{7} M_{\odot}$.

\subsubsection{UV Properties of $\mathrm{H \alpha}$ Non-detections}

As discussed in Paper I and Lee et al. (2009a), late-type galaxies that are completely devoid of star formation as traced by $\mathrm{H} \alpha$ emission are rare. In those papers, it was reported that $\mathrm{H} \alpha$ is not detected in only 22 of the 410 Local Volume galaxies that had been observed. Among these, 3 were classified as earlytype galaxies, and 19 as dwarf irregulars. All of the 19 late-type $\mathrm{H} \alpha$ non-detections have extremely low $B$-band luminosities $\left(M_{B} \gtrsim-13.6\right)$ and most also have some indication of the presence of H I gas. Therefore, we concluded that the lack of $\mathrm{H} \alpha$ emission might not necessarily indicate a lack of current star formation, but rather, may merely reflect the low probability of forming massive ionizing stars. This plausibility of this scenario can be tested with the current GALEX data set. Ongoing star formation that does not result in the production of ionizing stars and hence does not manifest itself via $\mathrm{H} \alpha$ emission should still be detectable with one-orbit-depth GALEX imaging. This is because B-stars down to $\sim 3 M_{\odot}$ significantly contribute to the FUV flux and are formed at much higher frequency than the more massive ionizing stars.

Of the 19 late-type $\mathrm{H} \alpha$ non-detections, 12 have observations which are at least one-orbit deep. ${ }^{24}$ False-color images of these

${ }^{24}$ UGCA15, LeoT, LSBCD564-08, ESO349-G031, UGC7298, UGCA276, M81dwA, LGS3, KDG73, KKR03, LSBCF573-01, and BK3N. galaxies are shown in Figure 6. All have measurable FUV emission except for UGCA276, which was discussed in the preceding section. These $11 \mathrm{H} \alpha$ undetected, UV-bright dwarf irregular galaxies are plotted in the color-magnitude diagrams in Figure 7 as the circled points. In contrast to Figure 3, only Sc and later-type spirals are now shown, and galaxies without $\mathrm{H} \alpha$ observations have been removed to focus the comparison. All 11 galaxies have blue UV colors typical of star-forming galaxies except for the two Local Group galaxies LGS3 and LeoT, which are the lowest-luminosity galaxies in the sample. The color of LeoT has too large of an error for the value to be meaningful, while LGS3's position off of the blue sequence may be an indication that star formation has not occurred in the past $\sim 100$ Myr. However, with a lifetime-averaged SFR of $\sim 5 \times 10^{-5} M_{\odot} \mathrm{yr}^{-1}$, even the UV emission of these systems is susceptible to Poisson noise in the formation of B-stars. ${ }^{25}$

It happens that observations of the resolved stellar populations of both LGS-3 (Miller et al. 2001) and Leo T (de Jong et al. 2008) do indicate the presence of recent star formation (within the last few hundred Myr). These studies of LGS-3 and Leo $\mathrm{T}$, together with the relatively blue UV colors of the remaining nine $\mathrm{H} \alpha$ non-detections, and the generally clumpy morphology of their FUV emission, provide evidence that the UV emission arises from stars that are a few hundred Myr old or younger, rather than from evolved stars as found in nearby elliptical galaxies (i.e., the "UV upturn;" O'Connell 1999, and references therein). If the observed FUV emission is translated into an $\mathrm{H} \alpha$ surface brightness (assuming the standard conversion of Kennicutt 1998b holds, and that the $\mathrm{H} \alpha$ and UV emission extend over the same regions of the disk), the expected values for all of the galaxies except LGS3 and LeoT are between $2 \times 10^{-17}$ and $1 \times 10^{-16}$ erg cm ${ }^{-2} \mathrm{~s}^{-1} \operatorname{arcsec}^{-2}$ (emission measures

\footnotetext{
25 Following Section 4.6 of Lee et al. (2009b), we can estimate when Poisson fluctuations may lead to FUV non-detections, by computing the SFR at which the number of OB stars at any given time would be $\sim 10$, as there will be times when a galaxy with said SFR will have $10-3 \sqrt{10}$ (i.e., zero) OB stars. Assuming a Salpeter IMF with mass range of $0.1-100 M_{\odot}, 95 \%$ of the FUV light is emitted by main-sequence stars between 3.3 and $100 M_{\odot}$. Thus, if the IMF is integrated between 3.3 and $100 M_{\odot}$, the mass of stars that must be formed in order to produce $10 \mathrm{OB}$ stars is $400 M_{\odot}$. To transform this into a SFR, the mass is divided by the median lifetime of stars that significantly contribute to the UV luminosity, which is $100 \mathrm{Myr}$. The limiting SFR is roughly $4 \times 10^{-4} M_{\odot} \mathrm{yr}^{-1}$. Monte Carlo simulations by Tremonti et al. (2007) confirm that Poisson fluctuations do not begin to significantly affect the FUV emission until SFRs of until at least $4 \times 10^{-4} M_{\odot} \mathrm{yr}^{-1}$.
} 

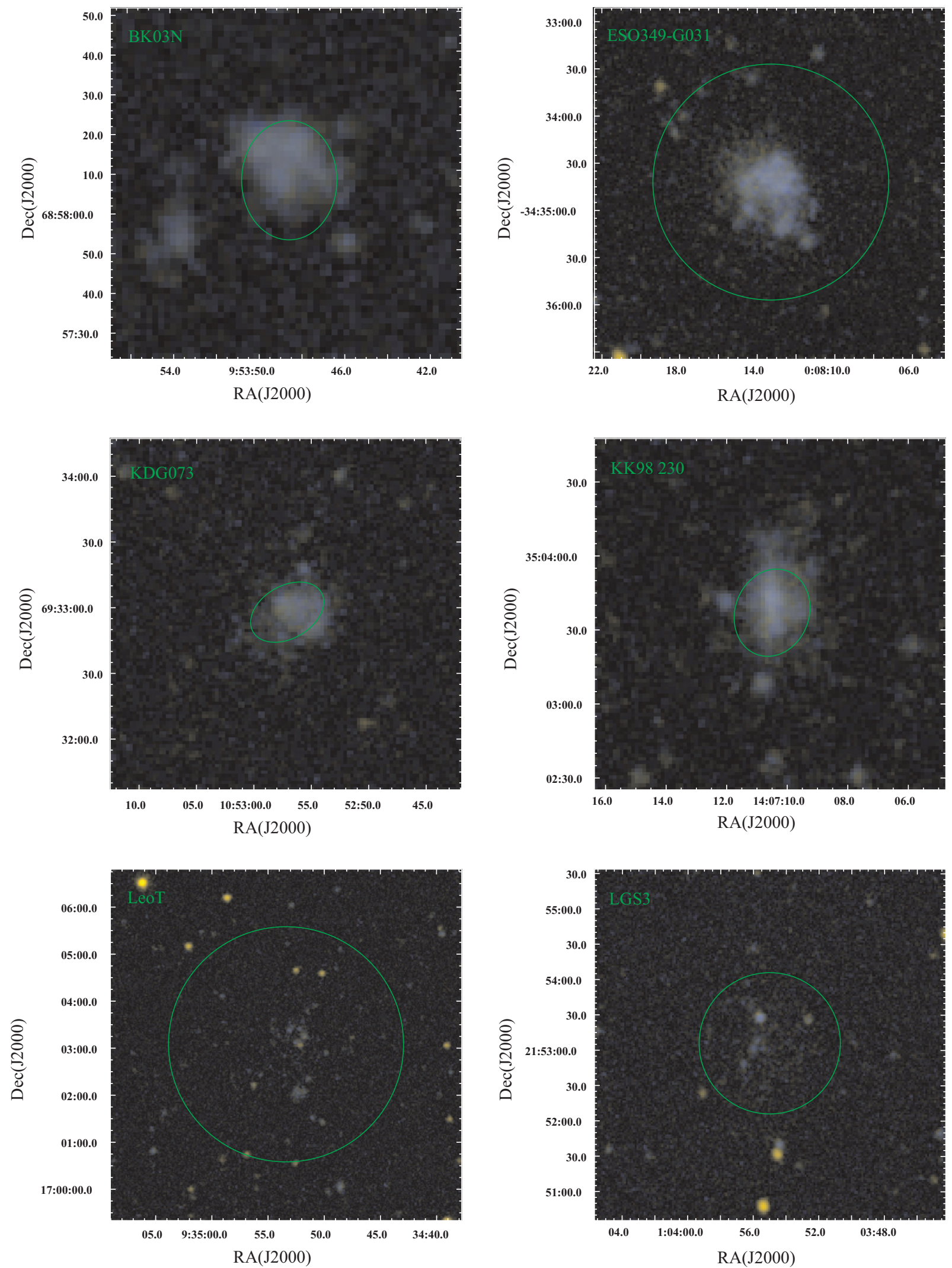

Figure 6. False-color images of late-type dwarf galaxies undetected in $\mathrm{H} \alpha$ narrowband imaging which have one-orbit-depth GALEX data ( 1500 s). The NUV emission appears as yellow and the FUV emission as blue. The green ellipses mark the RC $3 D_{25}$ ellipse. The FUV morphologies are generally clumpy suggesting that the UV emission arises from recent star formation.

(A color version of this figure is available in the online journal.) 

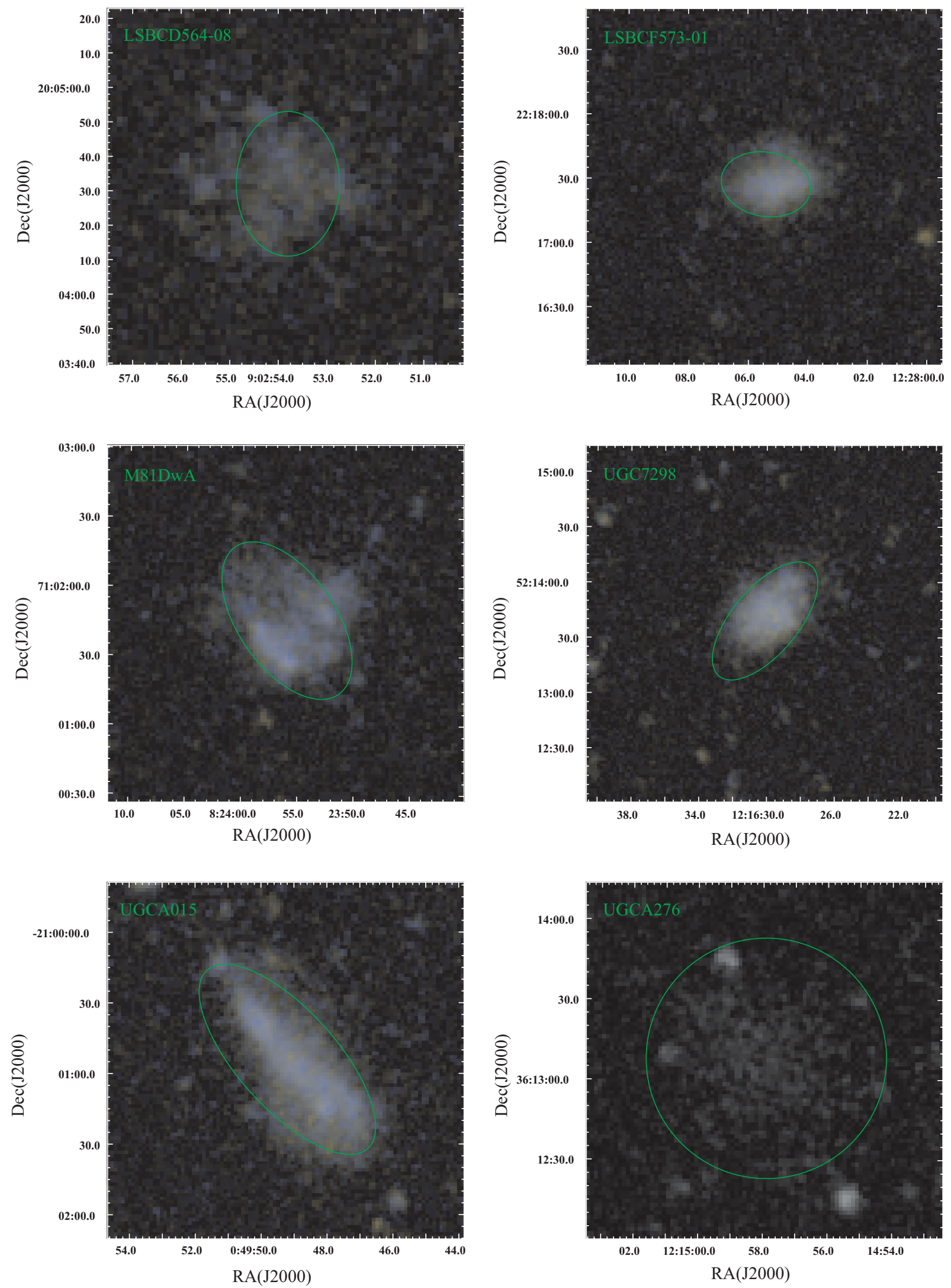

Figure 6. (Continued) 


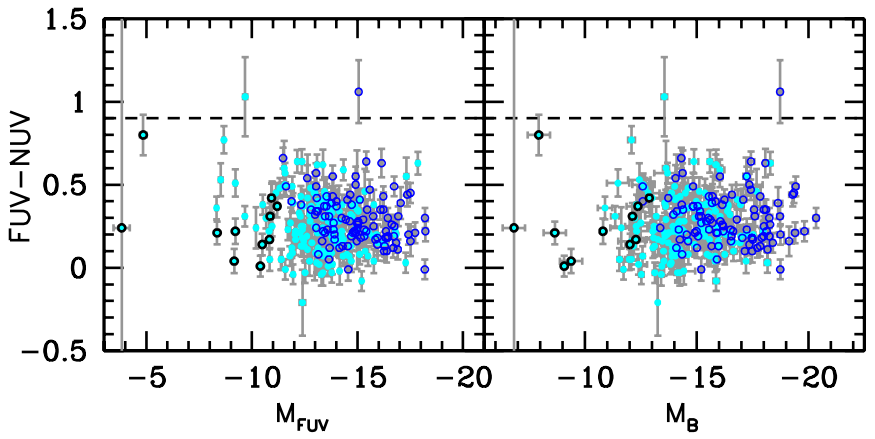

Figure 7. Same as the bottom panels of Figure 3, but with only Sc- and later-type galaxies shown. Only galaxies that have both FUV and $\mathrm{H} \alpha$ measurements are plotted. Errors are now also shown.

(A color version of this figure is available in the online journal.)

between $\sim 6$ and $30 \mathrm{pc} \mathrm{cm}^{-6}$ ). Therefore, the $\mathrm{H} \alpha$ non-detection of these galaxies should not be due to the limiting depth of the previous narrowband observations which probed to a $3 \sigma$ surface brightness of $\sim 1 \times 10^{-17} \mathrm{erg} \mathrm{cm}^{-2} \mathrm{~s}^{-1} \operatorname{arcsec}^{-2}$.

Given that the FUV emission likely arises from young stellar populations, the question then becomes whether the absence of $\mathrm{H}$ II regions is due to observing the galaxies during a particular time in their star formation histories when the most massive, ionizing stars have just died (i.e., star formation in the galaxy has recently halted), or whether it is merely the result of statistical fluctuations in the formation of massive stars in extremely lowmass systems (i.e., star formation is proceeding but massive stars have not been produced). Though it is difficult to unambiguously differentiate between these two scenarios with the current data set alone, we note that the $\mathrm{H} \alpha$-undetected, UV-bright dwarf irregular galaxies all have $M_{\mathrm{FUV}}>-11.2$ (Figure 7). The corresponding SFR is 0.0018 or $10^{-2.74} M_{\odot} \mathrm{yr}^{-1}$, which coincides with the SFR limit below which Poisson fluctuations in the formation of high-mass ionizing stars begin to affect the $\mathrm{H} \alpha$ output, as computed in Lee et al. (2009b). Thus, the UV data are at least not inconsistent with the possibility that the non-detection of $\mathrm{H} \alpha$ is due to stochasticity in the sampling of the massive end of the stellar IMF in systems with ongoing but ultra-low star formation activities. Again, a large, complete sample of these lowest-luminosity dwarf irregulars would be needed to probe this issue further. A new generation of recently developed stellar population synthesis models that incorporate a random sampling of a standard IMF (e.g., Eldridge 2010; Fumagalli et al. 2010; Popescu \& Hanson 2010) can be used to test whether the incidence of $\mathrm{H} \alpha$-undetected, UV-bright galaxies and the distribution of the $\mathrm{H} \alpha$-to-FUV flux ratio is consistent with the expectations of stochasticity, given a particular star formation history.

Another clue on the nature of star formation in $\mathrm{H} \alpha$ undetected, UV-bright dwarf irregular galaxies is provided by examining their UV-based star formation properties relative to those of the overall population of late-type galaxies. In Figures 4 and 5, where the SFR per unit Hi mass and stellar mass are plotted, the $\mathrm{H} \alpha$ non-detections are again circled in black. It is notable that most have global star formation efficiencies that are consistent with, and not highly deviant from, the distribution defined by the overall population. Aside from their extremely low luminosities, these galaxies appear to be relatively normal, at least by these global measures of the star formation activity. This picture is quite different from one based upon $\mathrm{H} \alpha$ observations alone. In fact, the non-detection of $\mathrm{H} \alpha$ in relatively gas-rich dwarf galaxies can potentially be used to identify "transition-type" systems, and this classification criterion was examined by Skillman et al. (2003). Transition-type systems are galaxies which have observed properties intermediate between dwarf spheroidals and dwarf irregulars, and thus, may be in a phase of transformation between the two galaxy classes (e.g., Mateo 1998; Grebel et al. 2003; Côté et al. 2009). In this context, the absence of $\mathrm{H} \alpha$ may be interpreted as an absence of the recent star formation, indicating that the galaxies are possibly evolving into red and dead spheroidals, or experiencing a temporary interruption of star formation due to effects within the group environment. However, galaxies classified as transition types also have very low luminosities. The analysis and discussion presented here clearly demonstrate that the nondetection of $\mathrm{H \alpha}$ in such extreme dwarfs is a highly insufficient criterion for identifying systems that are truly in a transformational state. An observation made by Skillman et al. (2003), that such transition types appear to be quite similar to dwarf irregulars (for example, in their spatial distribution within the group environment and in their gas properties), was suggestive of this conclusion. An absence of detectable FUV emission in gas-rich dwarfs would be a more compelling criterion, since the FUV flux is robust to stochastic fluctuations until much lower SFRs than $\mathrm{H} \alpha .{ }^{25}$ Alternately, an $\mathrm{H} \alpha$ non-detection accompanied by a red UV color (FUV - NUV $\gtrsim 0.6$ ) in such systems would also provide a better indication of halted star formation than the non-detection of $\mathrm{H} \alpha$ alone.

\section{SUMMARY}

The primary purpose of this paper is to provide GALEX NUVand FUV-integrated photometry for a statistically robust sample of star-forming galaxies within the $11 \mathrm{Mpc}$ Local Volume. Our GALEX programs have leveraged upon previously available observations to mitigate the previous $\sim 4$-fold underrepresentation of low-luminosity, nearby dwarfs with SFRs lower than $\sim 0.4 M_{\odot} \mathrm{yr}^{-1}\left(M_{\mathrm{FUV}} \gtrsim-14.5\right)$ in the GALEX archive. The resultant survey provides most complete UV imaging data set for Local Volume galaxies currently available. Together with the precursor $\mathrm{H} \alpha$ survey and the Local Volume Legacy infrared observations with Spitzer (as described in Kennicutt et al. 2008 and Dale et al. 2009, respectively), the GALEX observations reported here furnish a core data set for studying star formation and dust in the Galactic neighborhood.

We find that UV emission is present in nearly all of the late-type galaxies observed in our sample. This expected result supports the idea that the intermittent cessation of star formation is uncommon as we rarely observe such galaxies in an "off" mode (Lee et al. 2009a), at least for the $B<15.5$ population which is the main focus of our survey.

In the context of the recent work suggesting that FUV observations offer a more robust probe of star formation in lowdensity environments compared with previous $\mathrm{H} \alpha$ narrowband imaging, we use the data set to re-examine two measures of the global star formation efficiency, the SFR per unit H I gas mass and the SFR per unit stellar mass. We find that dwarf galaxies may not be as drastically inefficient in converting gas into stars as suggested by prior $\mathrm{H} \alpha$ studies, with the vast majority of galaxies in our sample having star formation efficiencies greater than $t_{\mathrm{H}}^{-1}$.

We also examine the properties of late-type dwarf galaxies that previously appeared to be devoid of star formation because they were not detected in earlier $\mathrm{H} \alpha$ narrowband observations. We find that such galaxies have UV SFRs that fall below the limit where the $\mathrm{H} \alpha$ output is expected to begin to suffer 
from Poisson fluctuations in the formation of massive stars. We note that the $\mathrm{H} \alpha$-undetected, UV-bright systems appear to be relatively normal with respect to the overall population of starforming galaxies in their UV-based star formation efficiencies and UV colors. Thus, the UV data are at least not inconsistent with the possibility that the absence of $\mathrm{H} \alpha$ is simply due to stochasticity in the sampling of the massive end of the stellar IMF, in otherwise normal star-forming systems with ongoing but ultra-low star formation activities.

At a number of points in the discussion, we remark that obtaining FUV and $\mathrm{H} \alpha$ observations for a deeper sample of dwarf galaxies, or more ideally, an $\mathrm{H}$ I-selected sample probing masses down to $10^{7} M_{\odot}$, is needed to test some of our conclusions. Such new observations are required to examine whether the rarity of star formation that has been temporarily halted on $\sim 100$ Myr timescales (i.e., the rarity of UV non-detections) persists in lower luminosity systems; test the apparent bound of star formation efficiencies to values greater than $t_{\mathrm{H}}^{-1}$; and to further probe whether the non-detection of $\mathrm{H} \alpha$ in UV-bright, $\mathrm{H}$ I-rich dwarf galaxies can be fully explained by Poisson sampling of a universal IMF.

GALEX is a NASA Small Explorer, and we gratefully acknowledge NASA's support for construction, operation, and science analysis for the GALEX mission, developed in cooperation with the Centre National d'Etudes Spatiales of France and the Korean Ministry of Science and Technology. This research has made use of the NASA/IPAC Extragalactic Database (NED), which is operated by the Jet Propulsion Laboratory, California Institute of Technology, under contract with NASA, as well as the HyperLeda database (http://leda.univ-lyon1.fr). We thank the anonymous referee for useful feedback which helped to clarify some important points in our interpretation of the data. Special thanks are due to Chris Martin and the members of the GALEX team (http://www.galex.caltech.edu/about/team.html) for their dedicated support of the Guest Investigator Program, without which this work would not have been possible. A.G.d.P. is supported by the Spanish "Ramón y Cajal", Plan Nacional AYA2009-10368, Consolider-GTC and AstroMadrid (CAM S2009/ESP-1496) programs.

Facilities: Bok, CTIO:0.9m, GALEX, Spitzer, VATT

\section{REFERENCES}

Adelman-McCarthy, J. K., et al. 2006, ApJS, 162, 38

Bigiel, F., Leroy, A., Walter, F., Brinks, E., de Blok, W. J. G., Madore, B., \& Thornley, M. D. 2008, AJ, 136, 2846

Blanton, M. R., Lupton, R. H., Schlegel, D. J., Strauss, M. A., Brinkmann, J., Fukugita, M., \& Loveday, J. 2005, ApJ, 631, 208

Boselli, A., Boissier, S., Cortese, L., Buat, V., Hughes, T. M., \& Gavazzi, G. 2009, ApJ, 706, 1527

Bothwell, M. S., Kennicutt, R. C., \& Lee, J. C. 2009, MNRAS, 400, 154

Brinchmann, J., Charlot, S., White, S. D. M., Tremonti, C., Kauffmann, G., Heckman, T., \& Brinkmann, J. 2004, MNRAS, 351, 1151

Cairós, L. M., Vílchez, J. M., González Pérez, J. N., Iglesias-Páramo, J., \& Caon, N. 2001, ApJS, 133, 321

Cardelli, J. A., Clayton, G. C., \& Mathis, J. S. 1989, ApJ, 345, 245

Côté, S., Draginda, A., Skillman, E. D., \& Miller, B. W. 2009, AJ, 138, 1037

Dalcanton, J. J., et al. 2009, ApJS, 183, 67

Dale, D. A., et al. 2009, ApJ, 703, 517

de Jong, J. T. A., et al. 2008, ApJ, 680, 1112

de Vaucouleurs, G., de Vaucouleurs, A., Corwin, H. G., Buta, R. J., Paturel, G., \& Fouque, P. 1991, Third Reference Catalog of Bright Galaxies, Vols. 1-3, XII (Berlin: Springer-Verlag)
Eldridge, J. J. 2010, arXiv:1008.1352

Fumagalli, M., da Silva, R., Krumholz, M., \& Bigiel, F. 2010, arXiv:1009.0524 Gallagher, J. S., III, \& Hunter, D. A. 1984, ARA\&A, 22, 37

Gil de Paz, A., \& Madore, B. F. 2005, ApJS, 156, 345

Gil de Paz, A., Madore, B. F., \& Pevunova, O. 2003, ApJS, 147, 29

Gil de Paz, A., et al. 2005, ApJ, 627, L29

Gil de Paz, A., et al. 2007, ApJS, 173, 185

Goddard, Q. E., Kennicutt, R. C., \& Ryan-Weber, E. V. 2010, MNRAS, 405, 2791

Grebel, E. K., Gallagher, J. S., III, \& Harbeck, D. 2003, AJ, 125, 1926

Hodge, P. W. 1974, PASP, 86, 845

Hunter, D. A., \& Elmegreen, B. G. 2004, AJ, 128, 2170

Hunter, D. A., Elmegreen, B. G., \& Ludka, B. C. 2010, AJ, 139, 447

Hunter, D. A., \& Gallagher, J. S., III. 1985, ApJS, 58, 533

James, P. A., Prescott, M., \& Baldry, I. K. 2008, A\&A, 484, 703

Karachentsev, I. D., \& Kaisin, S. S. 2007, AJ, 133, 1883

Karachentsev, I. D., Karachentseva, V. E., Huchtmeier, W. K., \& Makarov, D. I. 2004, AJ, 127, 2031

Karachentsev, I. D., \& Makarov, D. A. 1996, AJ, 111, 794

Kennicutt, R. C., Jr. 1983, ApJ, 272, 54

Kennicutt, R. C., Jr. 1989, ApJ, 344, 685

Kennicutt, R. C., Jr. 1998a, ApJ, 498, 541

Kennicutt, R. C., Jr. 1998b, ARA\&A, 36, 189

Kennicutt, R. C., Jr., Lee, J. C., Funes, S. J., José, G., Sakai, S., \& Akiyama, S. 2008, ApJS, 178, 247 (Paper I)

Kennicutt, R. C., Jr., Tamblyn, P., \& Congdon, C. E. 1994, ApJ, 435, 22

Knapen, J. H., \& James, P. A. 2009, ApJ, 698, 1437

Kroupa, P., \& Weidner, C. 2003, ApJ, 598, 1076

Lee, J. C., Kennicutt, R. C., Funes, S. J., José, G., Sakai, S., \& Akiyama, S. 2007, ApJ, 671, L113

Lee, J. C., Kennicutt, R. C., José, G., Funes, S. J., Sakai, S., \& Akiyama, S. 2009a, ApJ, 692, 1305

Lee, J. C., Salzer, J. J., \& Melbourne, J. 2004, ApJ, 616, 752

Lee, J. C., et al. 2009b, ApJ, 706, 599

Martin, D. C., et al. 2005, ApJ, 619, L1

Mateo, M. L. 1998, ARA\&A, 36, 435

Melena, N. W., Elmegreen, B. G., Hunter, D. A., \& Zernow, L. 2009, AJ, 138, 1203

Meurer, G. R. 2010, arXiv:1008.3946

Meurer, G. C., et al. 2006, ApJS, 165, 307

Meurer, G. R., et al. 2009, ApJ, 695, 765

Meyer, M. J., et al. 2004, MNRAS, 350, 1195

Miller, B. W., Dolphin, A. E., Lee, M. G., Kim, S. C., \& Hodge, P. 2001, ApJ, 562,713

Morrissey, P. 2006, Proc. SPIE, 6266, 26

Morrissey, P., et al. 2005, ApJ, 619, L7

Morrissey, P., et al. 2007, ApJS, 173, 682

O'Connell, R. W. 1999, ARA\&A, 37, 603

Paturel, G., Theureau, G., Bottinelli, L., Gouguenheim, L., Coudreau-Durand, N., Hallet, N., \& Petit, C. 2003, A\&A, 412, 57

Pflamm-Altenburg, J., \& Kroupa, P. 2009, ApJ, 706, 516

Pflamm-Altenburg, J., Weidner, C., \& Krapa, P. 2009, MNRAS, 395, 394

Popescu, B., \& Hanson, M. M. 2010, ApJ, 724, 296

Schiminovich, D., et al. 2007, ApJS, 173, 315

Schlegel, D. J., Finkbeiner, D. P., \& Davis, M. 1998, ApJ, 500, 525

Schmidt, M. 1959, ApJ, 129, 243

Skillman, E. D., Côté, S., \& Miller, B. W. 2003, AJ, 125, 593

Skillman, E. D., Kennicutt, R. C., \& Hodge, P. W. 1989, ApJ, 347, 875

Springob, C. M., Haynes, M. P., Giovanelli, R., \& Kent, B. R. 2005, ApJS, 160, 149

Thilker, D. A., et al. 2005, ApJ, 619, L79

Thilker, D. A., et al. 2007, ApJS, 173, 538

Tolstoy, E., Hill, V., \& Tosi, M. 2009, ARA\&A, 47, 371

Tremonti, C. A., Lee, J. C., van Zee, L., Kennicutt, R. C., Gil de Paz, A., Sakai, S., Funes, J., \& Akiyama, S. 2007, BAAS, 38, 894

Tully, R. B. 1988, AJ, 96, 73

Tully, R. B., et al. 2006, AJ, 132, 729

van Zee, L. 2001, AJ, 121, 2003

van Zee, L., Haynes, M. P., \& Salzer, J. J. 1997, AJ, 114, 2479

Weidner, C., \& Kroupa, P. 2005, ApJ, 625, 754

Weidner, C., \& Kroupa, P. 2006, MNRAS, 365, 1333

Weisz, D. R., Skillman, E. D., Cannon, J. M., Dolphin, A. E., Kennicutt, R. C., Jr., Lee, J., \& Walter, F. 2008, ApJ, 689, 160

Zaritsky, D., \& Christlein, D. 2007, AJ, 134, 135 